\title{
Effect of BLI-Type Inlet Distortion on Turbofan Engine Performance
}

\author{
James Redmond Lucas
}

Thesis submitted to the faculty of the Virginia Polytechnic Institute and State University in partial fulfillment of the requirements for the degree of

Master of Science

In

Mechanical Engineering

\author{
Walter F. O'Brien, Chair \\ Clinton L. Dancey \\ Alfred L. Wicks
}

June 12, 2013

Blacksburg, VA

Keywords: Performance, Boundary Layer Ingestion, Turbofan Engine, Inlet Distortion 


\title{
Effect of BLI-Type Inlet Distortion on Turbofan Engine Performance
}

\author{
James R. Lucas
}

\begin{abstract}
Boundary Layer Ingestion (BLI) is currently being researched as a potential method to improve efficiency and decrease emissions for the next generation of commercial aircraft. While reenergizing the boundary layer formed over the fuselage of an aircraft has many system level benefits, ingesting the low velocity boundary layer flow through a serpentine inlet into a turbofan engine adversely affects the performance of the engine. The available literature has only yielded studies of the effects of this specific type of inlet distortion on engine performance in the form of numerical simulations. This work seeks to provide an experimental analysis of the effects of BLI-type distortion on a turbofan engine's performance. A modified JT15D-1 turbofan engine was investigated in this study. Inlet flow distortion was created by a layered wire mesh distortion screen designed to create a total pressure distortion profile at the aerodynamic interface plane (AIP) similar to NASA's Inlet A boundary layer ingesting inlet flow profile. Results of this investigation showed a $15.5 \%$ decrease in stream thrust and a $14 \%$ increase in TSFC in the presence of BLI-type distortion.
\end{abstract}

Flow measurements at the AIP and the bypass nozzle exit plane provided information about the losses throughout the fan flow path. The presence of the distortion screen resulted in a $24 \%$ increase in mass-averaged entropy production along the entire fan flow path compared to the non-distorted test. A mass-averaged fan flow path efficiency was also calculated assuming an isentropic process as ideal. The non-distorted fan flow path efficiency was computed to be $60 \%$, while the distorted fan flow path efficiency was computed to be $50.5 \%$, a reduction in efficiency of $9.5 \%$. The entropy generation between ambient conditions and the AIP was compared to the entropy production along the entire fan flow path. It was found that the majority of entropy generation occurred between the AIP and bypass nozzle exit. Based on flow measurements at the bypass nozzle exit plane, it was concluded that inlet flow distortion should be located away from the tip region of the fan in order to minimize losses in a very lossy region. It was also determined that the fan and bypass duct process the different regions of the total pressure distortion in different ways. In some regions the entropy production decreased for the distorted test compared to the clean test, while in other regions the entropy production increased for the distorted test compared to the clean test. Finally, it was found that small improvements in total pressure and total temperature variation at the bypass nozzle exit plane will greatly improve the fan flow path efficiency and entropy generation, thereby decreasing performance losses. 


\section{Acknowledgements}

First, I would like to thank Virginia Tech and Pratt \& Whitney for providing me with financial support in the form of teaching assistantships and research assistantships. Without this support, I would not have been able to conduct this research.

I also wish to thank the members of my committee: Dr. Walter O'Brien, Dr. Clint Dancey, and Dr. Al Wicks. Thank you for your continued assistance and support throughout my time at Virginia Tech. I certainly could not have completed this degree without the help and encouragement each of you has provided.

I am extremely grateful to all of my friends and colleagues at the Virginia Tech Turbomachinery and Propulsion Research Lab: Tony Ferrar, Bill Schneck, Justin Bailey, Kevin Hoopes, Todd Pickering, Steven Steele, and Chaitanya Halbe. Working alongside each of you has been a pleasure and a privilege. I cannot thank you all enough for the support, encouragement, and assistance you have provided to me. Kevin, thank you for listening to my ideas and for allowing me to adjust your schedule so that I could "borrow" some of the equipment used in your experiments. Tony and Justin, thank you for allowing me to piggy-back on your experiments and for modifying your schedules to accommodate my needs. Your help in the manufacture and design of this experiment was instrumental in my success. Bill, thank you for the countless hours you have spent helping me derive equations, design this experiment, and organize my thoughts in a logical manner. Without all your efforts in LabVIEW programming and debugging electrical components this experiment would have never been completed. I cannot be more proud to be part of such a dedicated, hard-working, intelligent team, and I owe each of you my sincere gratitude.

To my parents, John and Jenny Lucas, and siblings, Joseph and Julia Lucas: I cannot begin to express my appreciation and gratitude for the love, support, and encouragement you have provided me. Thank you all for constantly believing in me and for pushing me to be the best I can be. Mom and Dad, I can only hope that one day my children will admire me as much as I do you.

To my newly acquired family: Lori Caldwell, Dwayne Wagner, Alex and Cindy Caldwell, and Emily Caldwell: Thank you for welcoming me and for loving and supporting me as a part of your family. I am blessed to have such a wonderful family-in-law.

Finally, to my wife, Hannah: thank you for being a constant source of love and encouragement. Thank you for bearing with me through the past few months and for continually supporting me in my quest for my Master's degree. You are my soul mate and best friend and I am so blessed to have you as my wife. I love you. 


\section{Table of Contents}

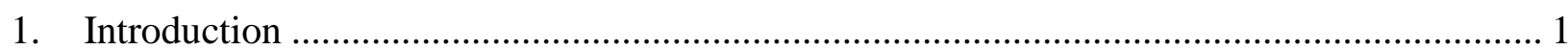

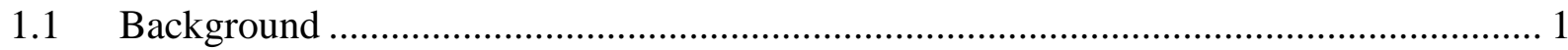

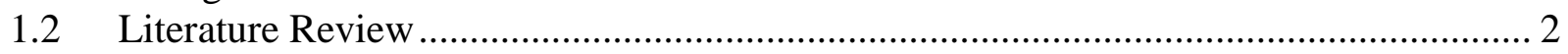

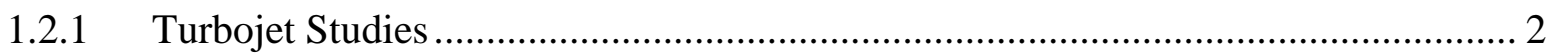

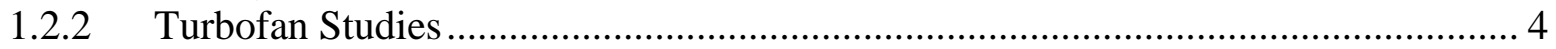

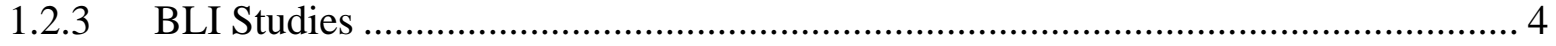

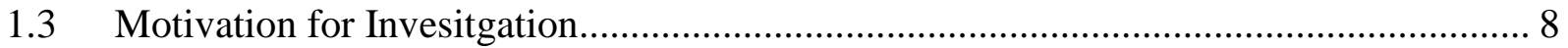

2. Experimental Methods....................................................................................... 10

$2.1 \quad$ Virginia Tech Distortion Test Facility ……………................................................. 10

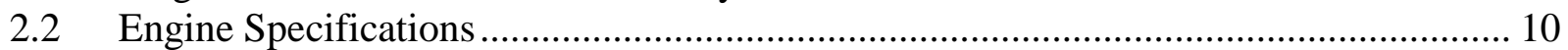

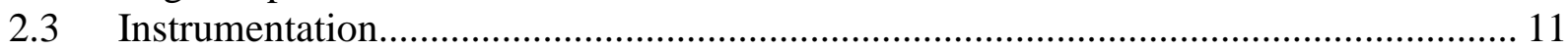

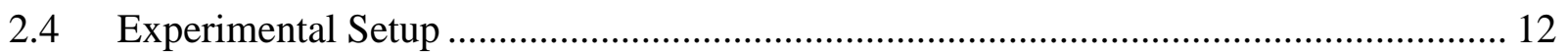

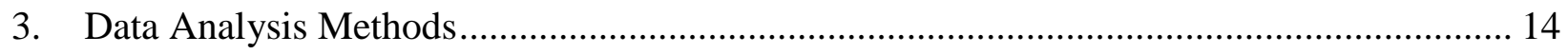

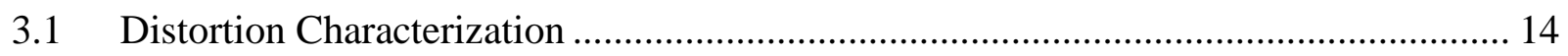

3.2 Engine Performance Calculations .......................................................................... 16

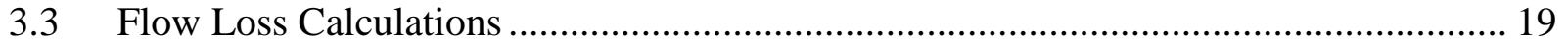

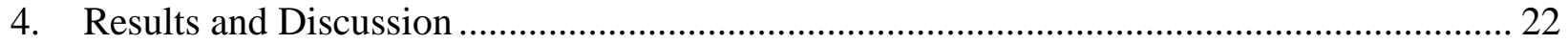

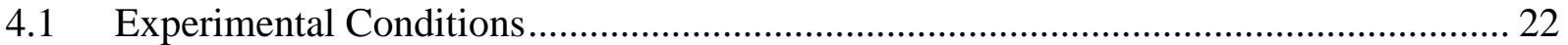

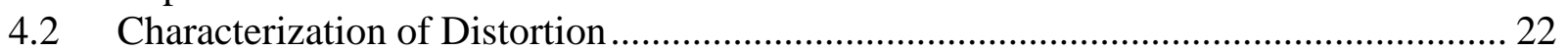

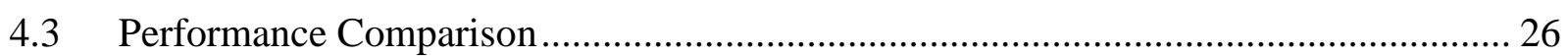

4.4 Performance Loss Analysis ..................................................................................... 27

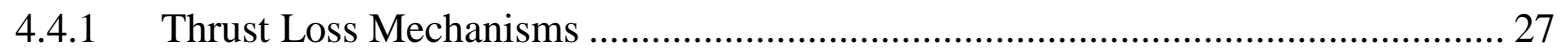

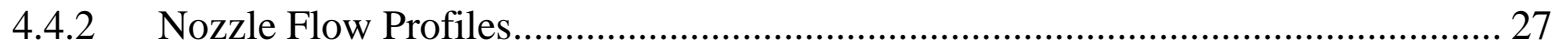

4.4.3 Distortion Attenuation ....................................................................................... 30

4.4.4 Entropy Generation and Efficiency ..................................................................... 33

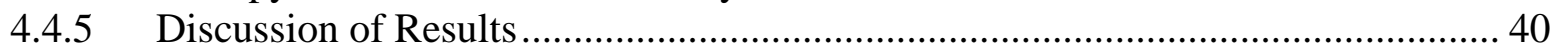

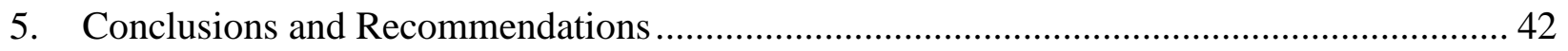

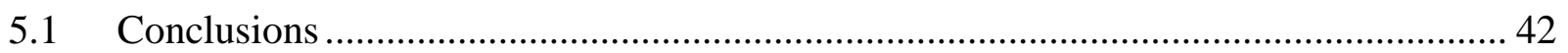

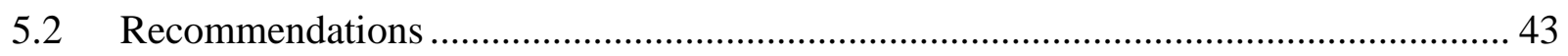

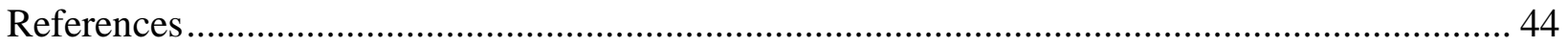

A. Derivation of Core Mass Flow Equations …………....................................................... 47

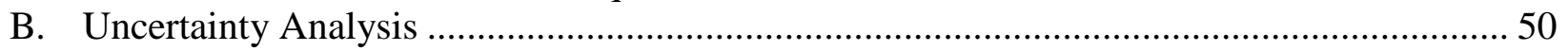

C. Proof of Constant Total Temperature Across Distortion Screen ............................................. 55 


\section{List of Figures}

Figure 1.1: Boundary Layer Ingesting Embedded Engine Flow Schematic.............................. 1

Figure 1.2: Typical BLI Engine Inlet Total Pressure Profile .............................................. 2

Figure 1.3: Effect of Fan Efficiency Losses on Typical UHB Engine TSFC ............................. 5

Figure 1.4: Effect of Inlet Total Pressure Losses on Typical UHB Engine TSFC ..................... 6

Figure 1.5: Total Pressure Profiles Upstream (Left) and Downstream (Right) of Fan................. 7

Figure 1.6: Static Pressure Profiles Upstream (Left) and Downstream (Right) of Fan ............... 7

Figure 2.1: Virginia Tech Inlet Distortion Test Facility ................................................... 10

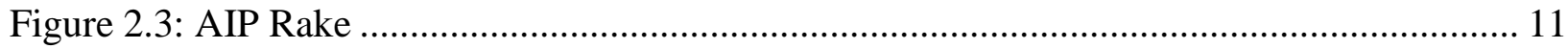

Figure 2.4: Bypass and Core Flow Measurement Probes ..................................................... 12

Figure 2.2: Backing Screen (Left) and Distortion Screen (Right) ....................................... 13

Figure 3.1: Sample Once-Per-Revolution Ring Total Pressure Profile with ARP1420 Distortion

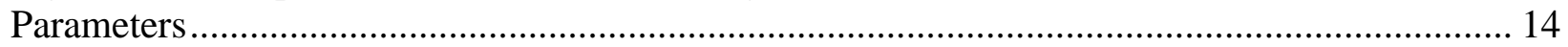

Figure 3.2: Stream Thrust Calculation Control Volume....................................................... 17

Figure 3.3: Flow Stations for Flow Loss Calculations ....................................................... 20

Figure 4.1: Contours of Total Pressure Recovery at AIP for (a) Clean Test and (b) Distorted Test

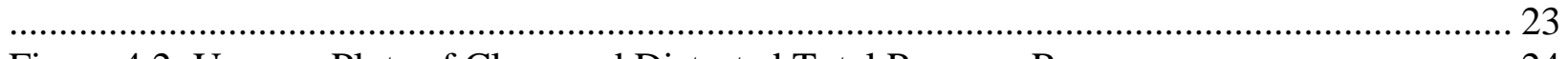

Figure 4.2: Unwrap Plots of Clean and Distorted Total Pressure Recovery ............................ 24

Figure 4.3: Circumferential Intensity at AIP for Distorted Test and Inlet A ........................... 25

Figure 4.4: Radial Intensity at AIP for Distorted Test and Inlet A ..................................... 25

Figure 4.5: Extent at AIP for Distorted Test and Inlet A.................................................... 25

Figure 4.6: Contours of Total Pressure Ratio at Bypass Nozzle Exit Plane for (a) Clean and (b)

Distorted Tests ........................................................................................................ 28

Figure 4.7: Contours of Normalized Static Pressure at Bypass Nozzle Exit Plane for (a) Clean and (b) Distorted Tests.............................................................................................. 29

Figure 4.8: Contours of Normalized Total Temperature at Bypass Nozzle Exit Plane for ......... 29

Figure 4.9: Ring Mean Normalized Total Pressure Profiles for Each Ring at AIP and Bypass

Nozzle Exit Plane for Distorted Test ................................................................................ 31

Figure 4.10: (a) Circumferential Intensity and (b) Extent at AIP and Bypass Nozzle Exit Plane

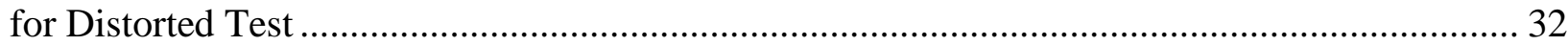

Figure 4.11: Percent Decrease in Circumferential Intensity from AIP to Bypass Nozzle Exit

Plane for Distorted Test .......................................................................................... 32

Figure 4.12: Flow Schematic for Entropy Generation and Efficiency Calculations ................... 33

Figure 4.13: Entropy Generated From Station 0 to Station 2 for Each Ring Measurement Stations

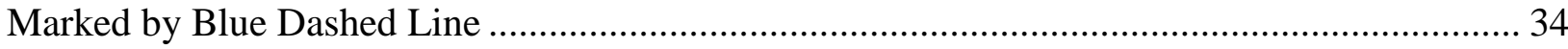

Figure 4.14: Entropy Generated from Station 0 to Station 1 for Ring 2 Measurement Stations

Marked by Blue Dashed Line ......................................................................................... 35

Figure 4.15: Entropy Production Change For Clean and Distorted Tests for Ring 2 ................. 36

Figure 4.16: Fan Flow Path Efficiency for Each Ring at the Bypass Nozzle Exit Plane ........... 38

Figure 4.17: Comparison of Raw Measurements with Calculated Fan Flow Path Efficiency and

Entropy Generation for Ring 2 for Distorted Test ........................................................... 39

Figure 4.18: Percent Variation in Pressure, Temperature, Entropy Generation, and Fan Flow

Path Efficiency at Bypass Nozzle Exit for Ring 2 Distorted Test ........................................ 40

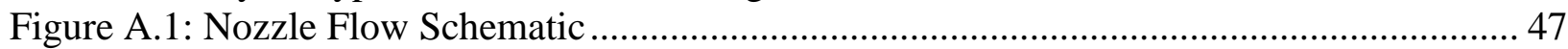


Figure B.1: Sample Error Bars for (a) AIP and (b) Bypass Nozzle Exit Plane Total Pressures... 51

Figure B.2: Fan Flow Path Entropy Production Uncertainty for Each Ring ........................... 53

Figure B.3: Fan Flow Path Efficiency Uncertainty for Each Ring ......................................... 53 


\section{List of Tables}

Table 2.1: JT15D-1 Design Specifications ....................................................................... 11

Table 4.1: Comparison of Conditions for Clean and Distorted Engine Tests ........................... 22

Table 4.2: Comparison of Performance Parameters for Clean and Distorted Tests ................... 26

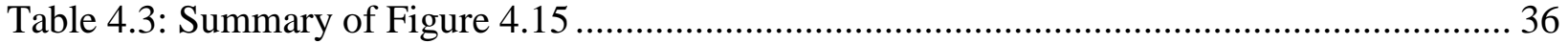

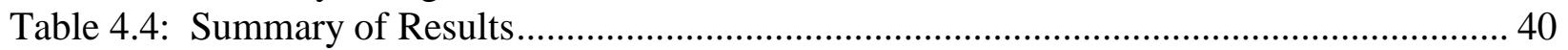

Table B.1: Measurement Uncertainty ......................................................................... 51

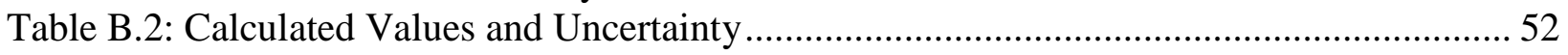

Table B.3: Effect of Specific Heat Capacity Assumption .................................................. 54 


\section{Nomenclature}

$\begin{array}{ll}\text { Alphanumeric Symbols } \\ A & \text { Area, } \mathrm{m}^{2} \\ M & \text { Mach number } \\ N & \text { Number of measurement rings } \\ P A V & \text { Ring average total pressure, } P a \\ P A V L O W & \text { Average total pressure in defect region, } P a \\ P F A V & \text { Measurement plane average total pressure, } P a \\ R & \text { Gas constant, } \frac{J}{\mathrm{kgK}} \\ R_{p} & \text { Local pressure recovery } \\ R_{p} & \text { AIP average pressure recovery } \\ T & \text { Static temperature, } K \\ T_{0} & \text { Total temperature, } K \\ T_{0, n o r m} & \text { Normalized total temperature } \\ T_{a t m} & \text { Ambient temperature, } K \\ T_{r e f} & \text { Reference temperature of } 288.15 \mathrm{~K} \\ & \\ c_{p} & \text { Specific heat at constant pressure, } \frac{\mathrm{J}}{\mathrm{kgK}} \\ \dot{m} & \text { Air mass flow rate, } \frac{\mathrm{kg}}{\mathrm{s}} \\ n & \text { Total number of measurement points } \\ p & \text { Static pressure, } P a \\ p_{0} & \text { Total pressure, } P a \\ p_{0, n o r m} & \text { Normalized total pressure } \\ p_{a t m} & \text { Ambient pressure, } P a \\ p_{r e f} & \text { Reference pressure of } 101325 \mathrm{~Pa} \\ \frac{\Delta p_{c}}{p} & \text { Circumferential distortion intensity } \\ \frac{\Delta p_{r}}{p} & \text { Radial distortion intensity } \\ \Delta s & \text { Entropy generation, } \frac{\mathrm{J}}{\mathrm{kgK}} \\ u & \text { Velocity, } \frac{\mathrm{m}}{\mathrm{s}} \\ & \end{array}$




\section{Greek Symbols}

$\Theta \quad$ Ratio of ambient temperature to reference temperature

$\mathrm{T}_{S} \quad$ Stream thrust, $N$

$\mathrm{T}_{s, \text { cor }}$ Corrected stream thrust, $N$

$\beta \quad$ Bypass ratio

$\gamma \quad$ Air specific heat ratio

$\delta \quad$ Ratio of ambient pressure to reference pressure

$\eta_{f f p} \quad$ Fan flow path efficiency

$\theta \quad$ Circumferential position, degrees

$\theta_{i}^{-} \quad$ Circumferential distortion extent, degrees

$\lambda \quad$ Nozzle total pressure ratio

\section{Subscripts}

$\begin{array}{ll}a & \text { Air } \\ b & \text { Bypass } \\ b e l l & \text { Bellmouth } \\ c & \text { Core } \\ e c & \text { Core exit plane } \\ e f & \text { Bypass exit plane } \\ f & \text { Fuel } \\ i & \text { Measurement point } \\ j & 1^{\text {st }} \text { station index for entropy generation calculation } \\ k & 2^{\text {nd }} \text { station index for entropy generation calculation }\end{array}$

\section{Abbreviations}

AIP Aerodynamic Interface Plane

BLI Boundary Layer Ingestion

HWB Hybrid Wing Body

TSFC Thrust Specific Fuel Consumption, $\frac{k g}{N s}$

$T S F C_{c o r}$ Corrected Thrust Specific Fuel Consumption, $\frac{k g}{N s}$

UHB Ultra High Bypass 


\section{Introduction}

\subsection{Background}

Non-uniform inlet flow, or inlet flow distortion, is a problematic phenomenon that occurs in numerous scenarios in propulsion turbine engines. These non-uniformities can be in the form of local total pressure and/or temperature variations, as well as swirling flow. The focus of this thesis will be on local total pressure variation, or total pressure distortion, and its effect on the performance of a turbofan engine.

Numerous total pressure distortion profiles can be produced in a multitude of flight scenarios. Flow separation within a serpentine inlet duct can create a localized total pressure loss. Highagility aircraft can also create total pressure distortion at the inlet to a propulsion turbine engine during rapid maneuvers. Strong crosswinds could lead to separation across the engine inlet cowling, creating a region of low total pressure within the inlet.

The total pressure distortion profile of interest for this investigation is that which is produced by the inlet flow path to a boundary layer ingesting (BLI) engine. Since the announcement of NASA's Environmentally Responsible Aviation goals to improve the efficiency and performance of future generations of commercial aviation, numerous studies have been conducted in attempts to develop the most efficient aircraft/engine combination. The focus of these studies has been on highly integrated vehicle designs optimized for best system level performance ${ }^{1}$. This has resulted in the emergence of a hybrid wing-body (HWB) vehicle in which the fuselage is designed to produce a significant portion of the overall lift. These vehicle configurations lend themselves to the option of embedding the engines at the rear of the fuselage such that they ingest the boundary layer that forms over the fuselage, as shown in Figure $1.1^{3}$. By ingesting and re-energizing the boundary layer that has formed over the fuselage of the aircraft, the aircraft's drag can be substantially reduced ${ }^{2}$.

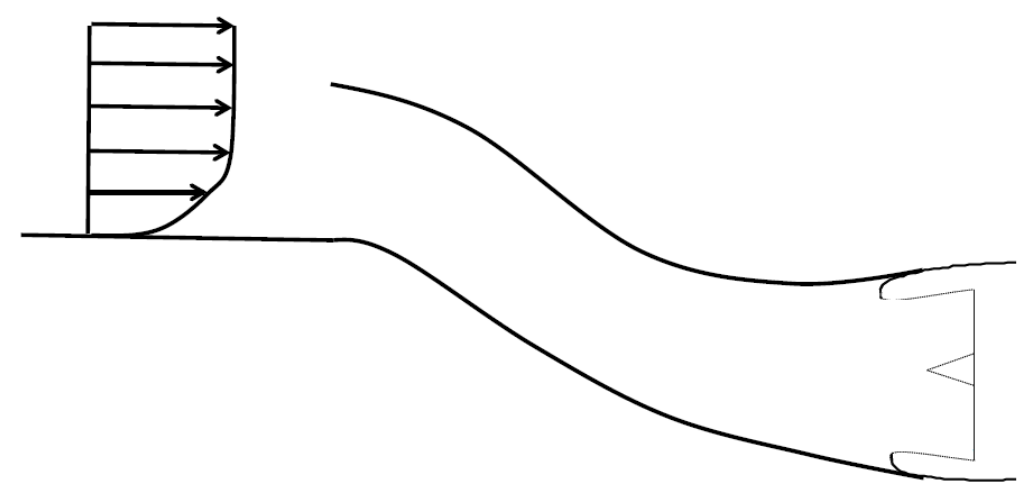

Figure 1.1: Boundary Layer Ingesting Embedded Engine Flow Schematic

The combination of the serpentine inlet duct and the lower velocity region of boundary layer flow results in a localized low total pressure region at the engine inlet. A typical embedded BLI inlet total pressure profile is shown in Figure $1.2^{3}$. 


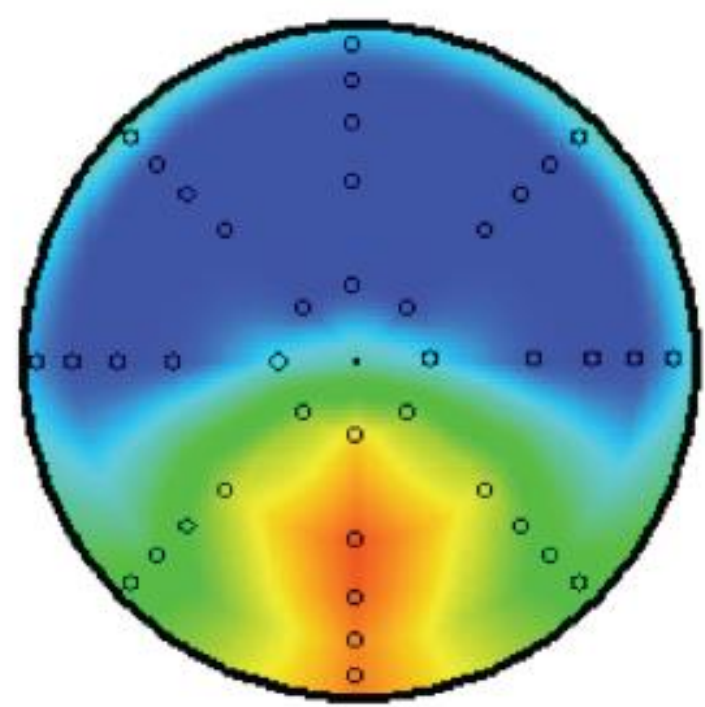

Figure 1.2: Typical BLI Engine Inlet Total Pressure Profile Red $=$ Low Total Pressure, Blue $=$ High Total Pressure

Local variations in flow total pressure can lead to many challenges in the operation of propulsion turbine engines. A once-per-revolution low total pressure region, such as the profile shown in Figure 1.2, can create fatigue issues due to cyclic loading and unloading as the turbomachinery rotates from a "uniform" high total pressure region to a low total pressure region, and then back to the "uniform" flow region. These fatigue issues are exacerbated in the presence of multipleper-revolution low pressure regions ${ }^{4}$.

In addition to aeromechanical problems, total pressure distortion is known to affect stability and the overall performance of jet engines. Localized low total pressure regions can lead to localized separation on fan/compressor blades, and eventually stall or surge. Thus, total pressure distortion shifts the engine's operating line towards the stall/surge line, decreasing efficiency of the fan/compressor, leading to a decrease in performance and an increase in fuel consumption for a given thrust requirement ${ }^{2}$. A review of previous studies that have investigated the performance penalty resulting from boundary layer ingestion as well as inlet flow distortion as a whole is presented in Section 1.2.

\subsection{Literature Review}

\subsubsection{Turbojet Studies}

The effect of inlet flow distortion on turbine engines has been an area of research since at least 19505. Between 1950 and 1968, numerous inlet distortion studies were performed on turbojet engines, as turbojet engines were predominantly used at that time while turbofan engines were in the early stages of development ${ }^{5,6,7,8}$. These tests sought to explain and analyze the performance changes of axial turbojet engines in the presence of generic radial and circumferential inlet pressure distortion screens in attempts to draw conclusions based on general trends. 
In a test conducted by Conrad, et $\mathrm{al}^{7}$, multiple circumferential, radial, and combined circumferential/radial distortion screens were inserted into the inlet duct of an axial turbojet engine. These screens created total pressure recoveries of $74 \%$ to $90 \%$ relative to atmospheric pressure. With all of these distortions, the performance of the engine was reduced, with $2-4 \%$ increases in thrust-specific fuel consumption (TSFC) for constant thrust requirements relative to non-distorted performance. For two distortion screens, namely an $87 \%$ pressure recovery radial screen and an $85 \%$ mixed circumferential/radial screen, stall/surge prevented the engine from reaching the thrust requirement, emphasizing the limitations on engine operability due to inlet distortion.

Russey and Seashore ${ }^{8}$ also performed an investigation into the performance change of an axial turbojet engine subjected to circumferential total pressure distortion. For a circumferential distortion screen with a single 70 degree sector resulting in a pressure recovery of $85 \%$, a $3.5 \%$ loss in thrust and a $1 \%$ increase in TSFC were experienced relative to non-distorted performance for constant corrected engine speed. Russey and Seashore determined that the losses in overall performance were likely linked to losses in the compressor, as the combustor and turbine efficiencies were unaffected by distortion while the compressor suffered a $2 \%$ loss in efficiency for the circumferential distortion described above. This study also found that the performance losses were greater at lower engine speeds. This phenomenon was attributed to the fact that the early compressor stages are more highly loaded at lower speeds. Thus, since the distortion hits the early compressor stages first, these already highly loaded stages will be even more highly loaded in the presence of distortion, leading to performance decreases.

Not only did the early turbojet engine studies conduct performance analyses in the presence of distortion, they also presented some common results regarding the transmission of distortion through the engine. One result that is found in each of these studies is that total pressure distortion tends to attenuate through a multi-stage compressor ${ }^{5,6,7,8,9}$. Conrad, et al. measured the total pressure profile behind the fourth compressor stage in a 12-stage axial turbojet engine and found that the total pressure distortion present at the compressor inlet was almost entirely eliminated after the fourth compressor stage for multiple types of distortion ${ }^{7}$.

A second result shared by the turbojet engine distortion performance studies is that the total pressure distortion creates a total temperature distortion that is transmitted through the entire turbojet engine $e^{5,7,8}$. Conrad, et al found that total temperature variations as high as $9 \%$ can be found at the turbine exit. These temperature variations could create problems in the turbine, as the turbine blade materials are pushed close to their temperature limits during normal operation. Local increases in temperature could create increased stresses in a turbine blade, decreasing its lifetime and performance ${ }^{7}$. The opposite transmission trends of total pressure and temperature distortions through compressors were explained by Plourde and Stenning ${ }^{9}$, who sought to explain the attenuation of circumferential total pressure distortion through a compressor using a mathematical model. The results of this model led to the conclusion that the attenuation of total pressure distortion is due to the negative slope of the compressor pressure rise vs. flow rate characteristic. Compressors do more work in low velocity, low pressure regions than in high velocity, high pressure regions, which tends to smooth out and attenuate the total pressure distortion as it passes through the compressor. The slope of the compressor pressure rise vs. 
flow rate characteristic is also the reason that total temperature distortions are created and exacerbated through compressors. The increased work done by the compressor in low pressure, low velocity regions leads to higher temperatures in those regions than in the high pressure, high velocity regions. Thus, while the total temperature at the compressor inlet is fairly uniform, large temperature variations will be created in the flow as it passes through the compressor.

\subsubsection{Turbofan Studies}

The author has only found three available studies on turbofan engines from which information related to this thesis could be gleaned. The first of these is a study performed by Walter and Shaw ${ }^{10}$ in which an expanded parallel compressor model was used to predict the response of a F100 turbofan engine to circumferential total pressure distortion. This study was primarily focused on the attenuation of the distortion through the fan and the effects of the distortion on the stability of the fan. Walter and Shaw found that for both 90 degree and 180 degree circumferential distortions with $22 \%$ pressure variation relative to the mean total pressure, about $10 \%$ of the distortion was attenuated across the 3 -stage fan in the F100. These $22 \%$ pressure variation distortions also resulted in about $7 \%$ to $8 \%$ variation in total temperature downstream of the fan. These results give credence to the findings of Plourde and Stenning, since a fan is in essence a compressor. This study also found that surge margin decreases substantially with distortion.

Soeder and Bobula ${ }^{11}$ performed a flow propagation study on a YTF34 turbofan engine subjected to 90 degree and 180 degree circumferential distortions with $8 \%$ to $10 \%$ total pressure variation relative to the mean total pressure. This study found that both total and static pressure variations were completely attenuated through the compressor, further confirming the findings of Plourde and Stenning.

Finally, Katsiopoulos, et $\mathrm{al}^{12}$ performed a performance prediction of a J79-GE-17 turbofan engine using a parallel compressor theory model. This study calculated the engine's operating line and surge line under a distortion with an extent of 90 degrees and an intensity of 0.15 using ARP $1420^{19}$ distortion descriptors. This study predicted a $2 \%$ loss of thrust and an $8 \%$ loss in surge margin compared to non-distorted performance for an engine in mint condition. This study also incorporated effects of degradation of engine components due to wear and tear and predicted an $8 \%$ loss of thrust and $100 \%$ loss of surge margin for an engine with typically degraded components. Thus, according to parallel compressor theory, the condition of engine components can significantly affect performance of an engine subjected to distortion.

\subsubsection{BLI Studies}

Many studies have been performed on hybrid wing-body airframe/engine systems focusing on overall mission performance in attempts to quantify the potential benefits of using hybrid wingbody aircraft with boundary layer ingesting engines. Due to the conceptual nature of these hybrid wing-body aircraft, all of the literature the author has found regarding the performance of BLI engines has been in the form of theoretical models and simulations. 
In 2003, Daggett, et a ${ }^{13}$ performed a full system analysis of a Boeing Blended Wing Body with GE58 ultra high bypass ratio turbofan engines. In this study, the GE58 engine performance was modeled in Boeing's EDASA performance modeling program. The effect of the BLI distortion on the GE58 performance was simulated as a loss in inlet pressure recovery. The EDASA code estimated that by increasing the amount of boundary layer ingested by the engines, the thrust loss as a result of inlet pressure recovery losses could be $10 \%$ or greater.

Many other studies have attempted to model the effect of BLI-type distortion on engine performance as a loss in fan efficiency. Tillman ${ }^{14}$ estimates that BLI-type distortion creates a loss of fan efficiency of at least $2 \%$, but also states that with careful inlet duct design to minimize the BLI distortion, the fan efficiency loss can be reduced to about $1 \%$.

Hardin, et $\mathrm{a}^{15}$ used an NPSS model of a typical ultra-high bypass (UHB) turbofan engine to determine performance changes due to BLI-type distortion. To account for performance losses due to reduced fan efficiency and increased inlet total pressure losses, the engine's TSFC sensitivity to various values of fan efficiency and inlet total pressure recovery was calculated in NPSS. These sensitivity curves are shown in Figure 1.3 and Figure $1.4^{15}$.

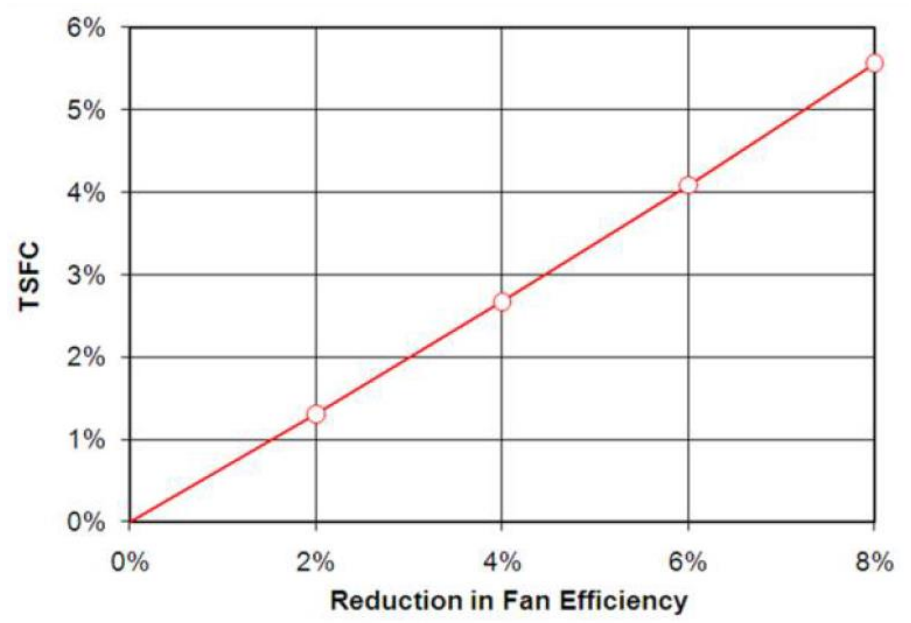

Figure 1.3: Effect of Fan Efficiency Losses on Typical UHB Engine TSFC in Hardin's Study 


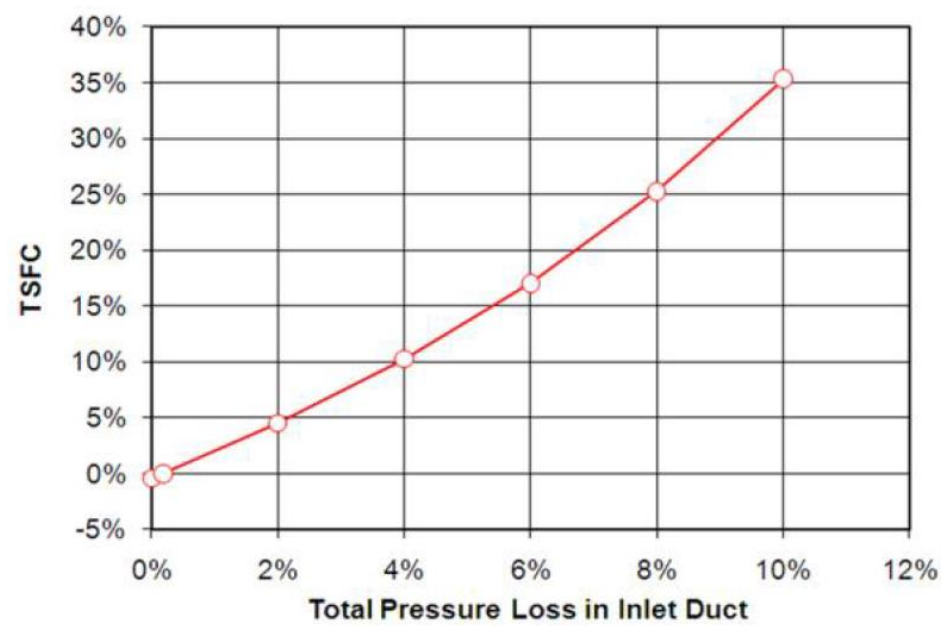

Figure 1.4: Effect of Inlet Total Pressure Losses on Typical UHB Engine TSFC in Hardin's Study

Based on the results shown in Figure 1.3 and Figure 1.4, TSFC is much more sensitive than fan efficiency to inlet total pressure losses, but both inlet total pressure losses and fan efficiency losses have a substantial negative impact on TSFC. Hardin, et al. then proceeded to model the flow over a typical HWB with BLI engines, using the sensitivity curves shown above to determine TSFC losses in the presence of BLI-type distortion. This study found that BLI caused a $0.35 \%$ inlet total pressure loss and a $1.3 \%$ fan efficiency loss, each resulting in a $1.1 \%$ fuel burn increase and a $0.86 \%$ TSFC increase. Thus, combining the effects of inlet total pressure losses and fan efficiency losses, fuel burn increased by $2.2 \%$ and TSFC increased by $1.72 \%$ in the presence of BLI-type inlet distortion ${ }^{15}$.

Gladin $^{17}$, et al used physics-based multidisciplinary simulation software to model a HWB 300 passenger aircraft with UHB geared turbofan engines in both podded and BLI configurations. Based on the results of previous studies ${ }^{14,16}$, Gladin modeled the effect of BLI distortion on engine performance as decreases in fan efficiency of $1 \%$ to $3 \%$ and decreases in inlet total pressure loss of $1 \%$ to $2 \%$. With these losses included in the models, the engines' TSFC increased between $1 \%$ with the lower losses and $4 \%$ with the higher losses.

To date, the author has only found two studies in which the interaction of an engine and the nonuniform inlet flow caused by BLI was investigated. In the first of these studies, conducted by Plas, et $\mathrm{al}^{18}$, a three-dimensional body-force based model was used to simulate fan response to BLI-type inlet distortion. The results of this model estimated a $1 \%$ fan efficiency loss due to BLI-type distortion. In addition to fan performance calculations, the total and static pressure profiles upstream and downstream of the fan were calculated. These profiles are shown in Figure 1.5 and Figure $1.6^{18}$. 

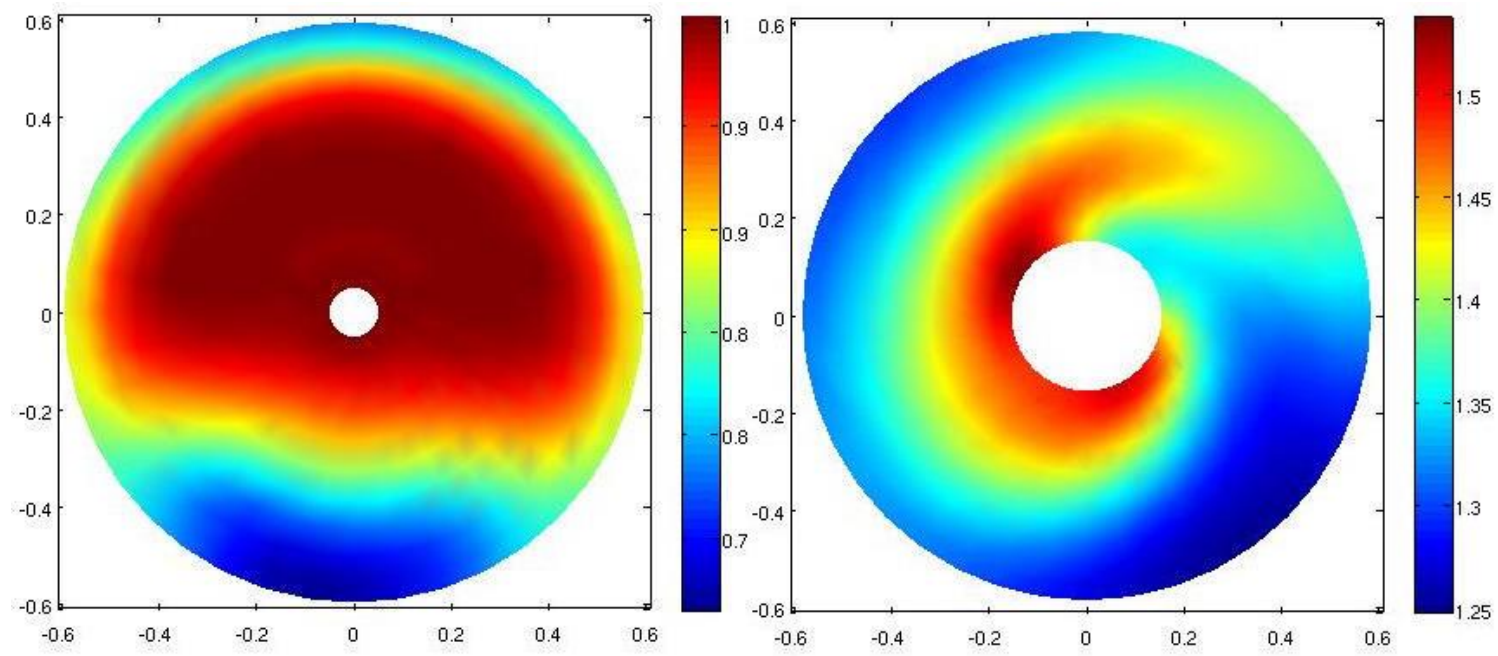

Figure 1.5: Total Pressure Profiles Upstream (Left) and Downstream (Right) of Fan in Plas's Study
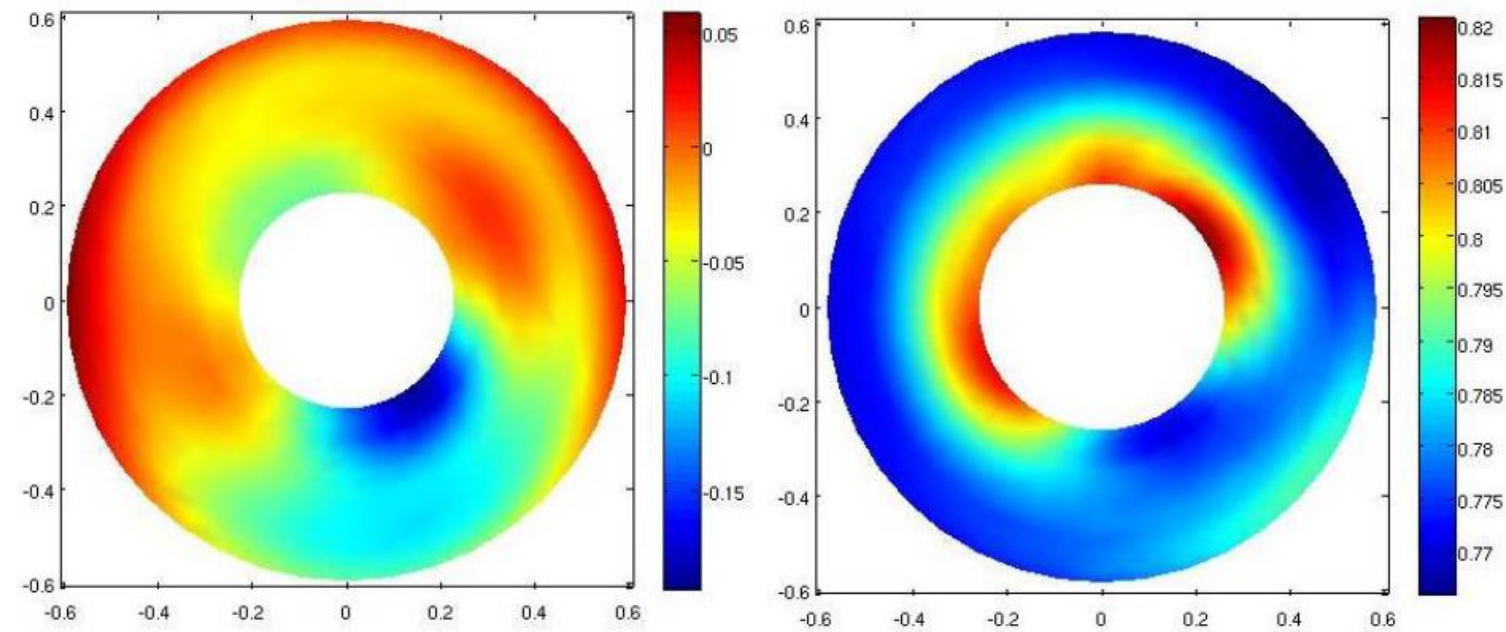

Figure 1.6: Static Pressure Profiles Upstream (Left) and Downstream (Right) of Fan in Plas's Study

It is important to note that not only does the total pressure distortion persist downstream of the fan, but static pressure distortion persists downstream of the fan as well. This leads to problems with parallel compressor theory models, in which it is assumed that the static pressure downstream of a fan is constant. The results of the model created by Plas, et al suggest that results obtained using parallel compressor theory are not entirely accurate for this application ${ }^{18}$.

In the second study of fan/distortion interaction, conducted by Yao, et al. ${ }^{24,25}$, CFD was used to predict the distortion transfer across a multistage fan. In addition to proving the capability of CFD to accurately match experimental results, a focus of this study was on the total pressure and total temperature profiles downstream of the fan. The total pressure profile upstream of the fan that was simulated in this study is shown in Figure 1.7, and the resulting total pressure and total temperature profiles downstream of the first fan stage are shown in Figure 1.8. 


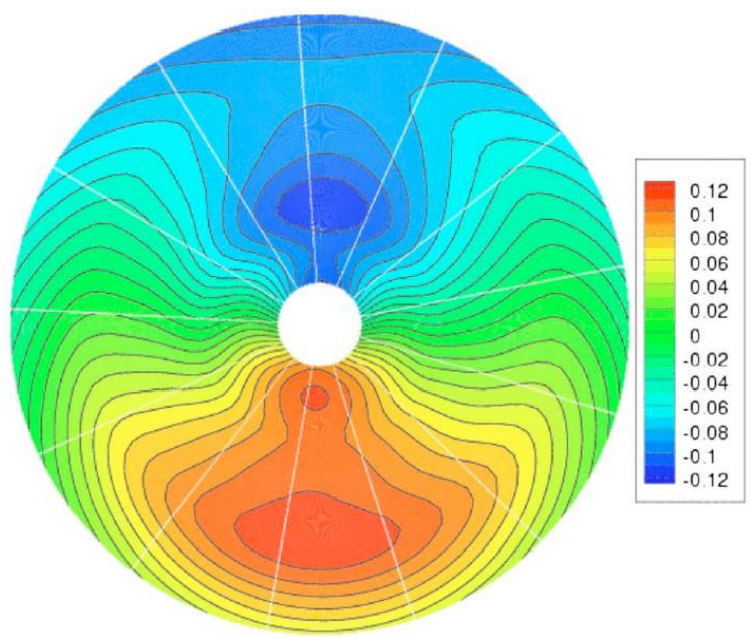

Figure 1.7: Total Pressure $\left[\frac{p_{0}-\overline{p_{0}}}{\overline{p_{0}}}\right]$ Profile Upstream of Fan in the Yao, et al. Study
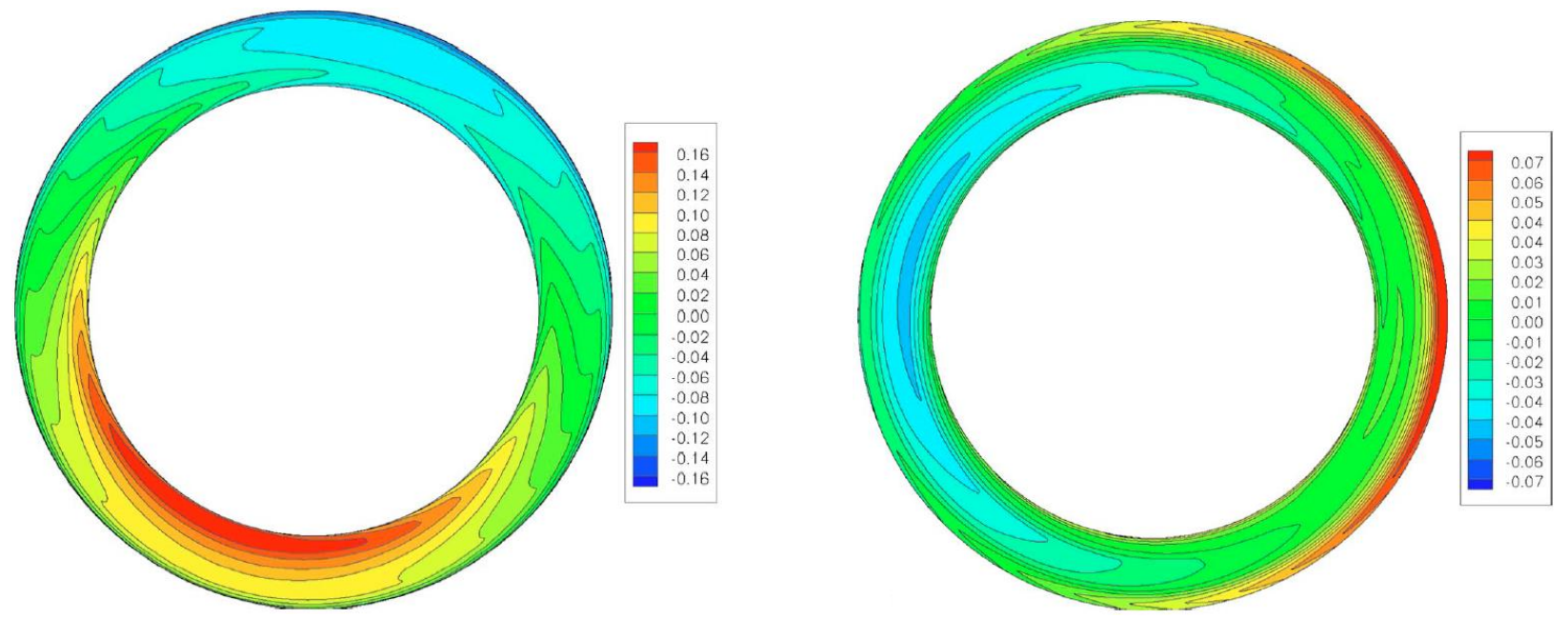

Figure 1.8: (a) Total Pressure $\left[\frac{p_{0}-\overline{p_{0}}}{\overline{p_{0}}}\right]$ and (b) Total Temperature $\left[\frac{T_{0}-\overline{T_{0}}}{\overline{T_{0}}}\right]$ Profile Downstream of First Fan Stage in the Yao, et al. Study

As shown in Figure 1.7 and Figure 1.8, a total pressure profile similar to that of an embedded BLI engine system upstream of a fan results in total pressure and total temperature distortion downstream of the first fan stage. Upon comparison of the total pressure and total temperature profiles in Figure 1.8, a phase shift exists between the total pressure profile and the total temperature profile.

\subsection{Motivation for Investigation}

As mentioned previously, there is very little available literature detailing distortion performance analyses similar to those described in Section 1.2.1 for modern turbofan engines. This is likely the result of a number of factors. First, testing jet engines is expensive, and the cost has increased with advanced engine technology and designs. Not only are the engines themselves 
very expensive, the fuel necessary to run the jet engine is expensive as well. Conducting performance comparisons of engines subjected to distorted inlet flow versus clean inlet flow means running an engine at least twice if the experiment is well designed and everything runs smoothly for each experiment. Possible malfunctions in instrumentation or data collection, or inconclusive data could lead to numerous more engine runs, each of which is not cheap.

Second, distortion tests on jet engines are dangerous. As described above, the presence of distortion increases blade stresses and creates surge/stall problems, each of which could potentially destroy a very expensive jet engine.

A third possible reason for the lack of available literature describing performance analyses of turbofan engines subjected to inlet distortion is that inlet distortions can be created in military flight applications. This would lead to the classification, and therefore unavailability, of any experiments that would be related to military applications.

Fourth, the increase in computational ability has likely led to a decline in physical testing of jet engines. It is significantly cheaper, safer, and sometimes faster to perform computational analyses than it is to perform an experiment on an actual engine. With the development of programs like NPSS, propulsion system simulations are easier to perform and more accurate.

Finally, for commercial applications, there has been little need or desire to investigate the effects of inlet distortion. Modern commercial engines are designed to tolerate little distortion in an attempt to maximize performance. For the most part, these engines are only subjected to transient distortions during relatively short, off-design operations. For this reason, most of the previous investigations into the effect of inlet distortion on engines have focused on stability of the fan/compressor to ensure the engine will recover from transient inlet distortion. BLI systems, on the other hand, will subject the engines to distortion throughout the entire operation of the aircraft. Thus, a detailed, physical investigation of the performance of an engine subjected to distortion typical of BLI systems with serpentine inlets is warranted. It is the purpose of this thesis to investigate the engine performance penalty associated with distorted inflow typical of an embedded BLI engine inlet system. Corrected thrust and TSFC will be the primary performance metrics used to characterize the performance changes due to this type of inlet distortion while other measurements and calculations such as bypass ratio, nozzle efficiency, and flow profiles will be used to explain the causes of the performance changes. 


\section{Experimental Methods}

\subsection{Virginia Tech Distortion Test Facility}

The engine distortion response test stand at the Virginia Tech Turbomachinery and Propulsion Research Laboratory was used to analyze the change in performance of a turbofan engine with distorted inlet flow. The test stand allows for a calibrated distortion generating device, such as a screen, to be positioned upstream of the engine. A CAD model of the test stand is shown in Figure 2.1.

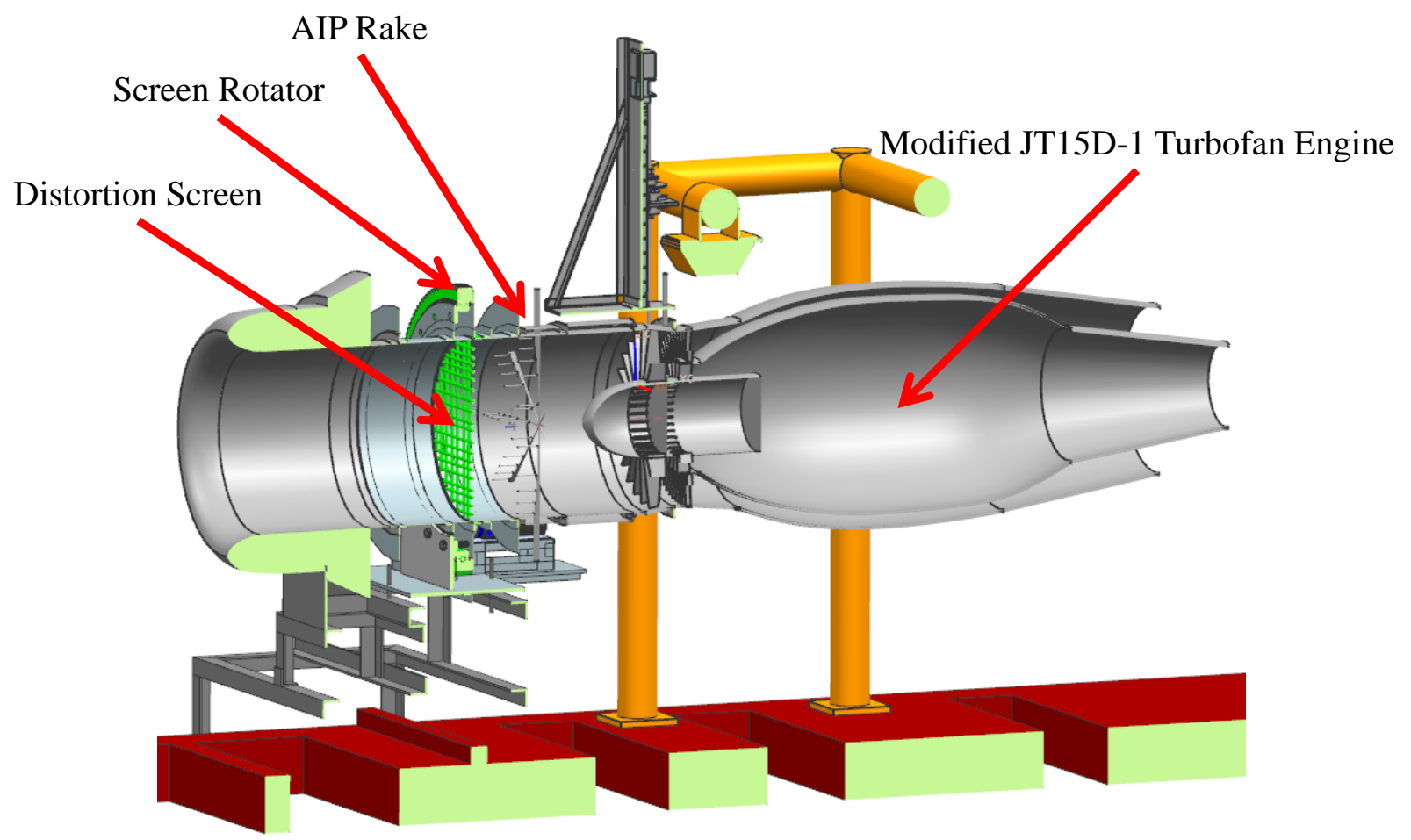

Figure 2.1: Virginia Tech Inlet Distortion Test Facility

\subsection{Engine Specifications}

A modified Pratt and Whitney Canada JT15D-1 turbofan engine was used for this study. This engine has a transonic fan, a single stage centrifugal compressor, reverse flow combustors, a single stage high pressure turbine, and a single stage low pressure turbine. Table 2.1 presents a summary of the design and performance specifications of the JT15D-1. 
Table 2.1: JT15D-1 Design Specifications

\begin{tabular}{|c|c|}
\hline Parameter & Value \\
\hline Fan 100\% RPM & 16000 \\
\hline Engine Mass Flow [lbm/s] & 73 \\
\hline Bypass Ratio & 3.3 \\
\hline Fan Pressure Ratio & 1.5 \\
\hline Compressor Pressure Ratio & 8 \\
\hline Fan Diameter [in] & 21 \\
\hline Thrust (SL, Take-off) [lbf] & 2200 \\
\hline TSFC (SL, Take-off) [lbm/s/lbf] & 0.54 \\
\hline
\end{tabular}

\subsection{Instrumentation}

The engine shown in Figure 2.1 was instrumented with a calibrated bellmouth inlet to measure the mass flow of air entering through the engine inlet. The inlet mass flow was measured using a calibration curve and 4 static pressure taps spaced equally around the inlet duct circumference.

A 60-probe total pressure rake was installed behind the distortion screen at the Aerodynamic Interface Plane (AIP) to measure the total pressure profile entering the engine. The AIP rake consisted of 12 rake arms with 5 total pressure probes on each arm. The probes on each arm were arranged such that they measured the total pressure of the flow at the mid-radius of equalarea rings within the inlet duct. The AIP rake, shown in Figure 2.2, was installed 0.67 diameters upstream of the fan during all test cases.

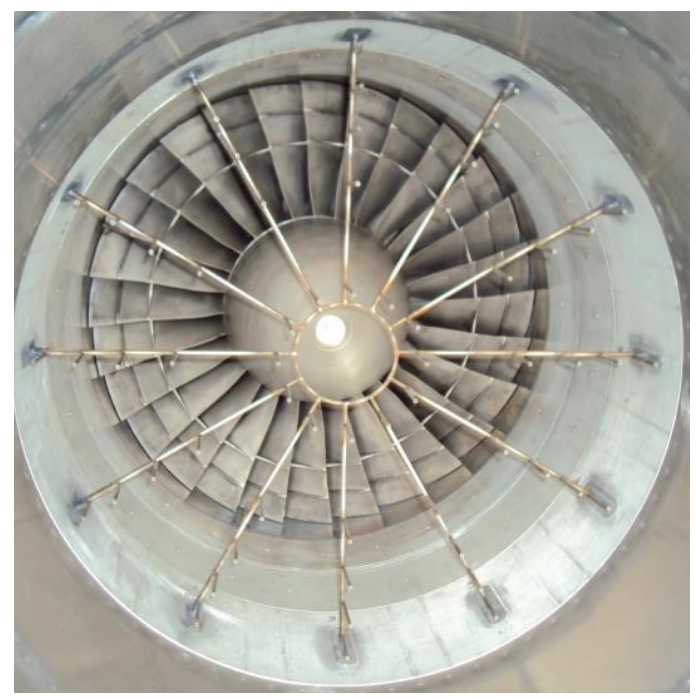

Figure 2.2: AIP Rake

In order to analyze the performance of the turbofan engine subjected to inlet distortion, the flow exiting the engine needed to be measured. To measure the bypass flow profile, A United Sensor pitot-static probe with a thermocouple, hereafter referred to as the bypass traversing probe, was installed in a probe traverse at the bypass nozzle exit plane. The core flow characteristics were 
measured using a static pressure tap located at the core nozzle inlet plane and a thermocouple protruding into the core nozzle flow located near the nozzle exit plane. Figure 2.3 shows the orientation and location of the bypass and core flow measurement probes. A more detailed performance analysis description can be found in Section 3.2.

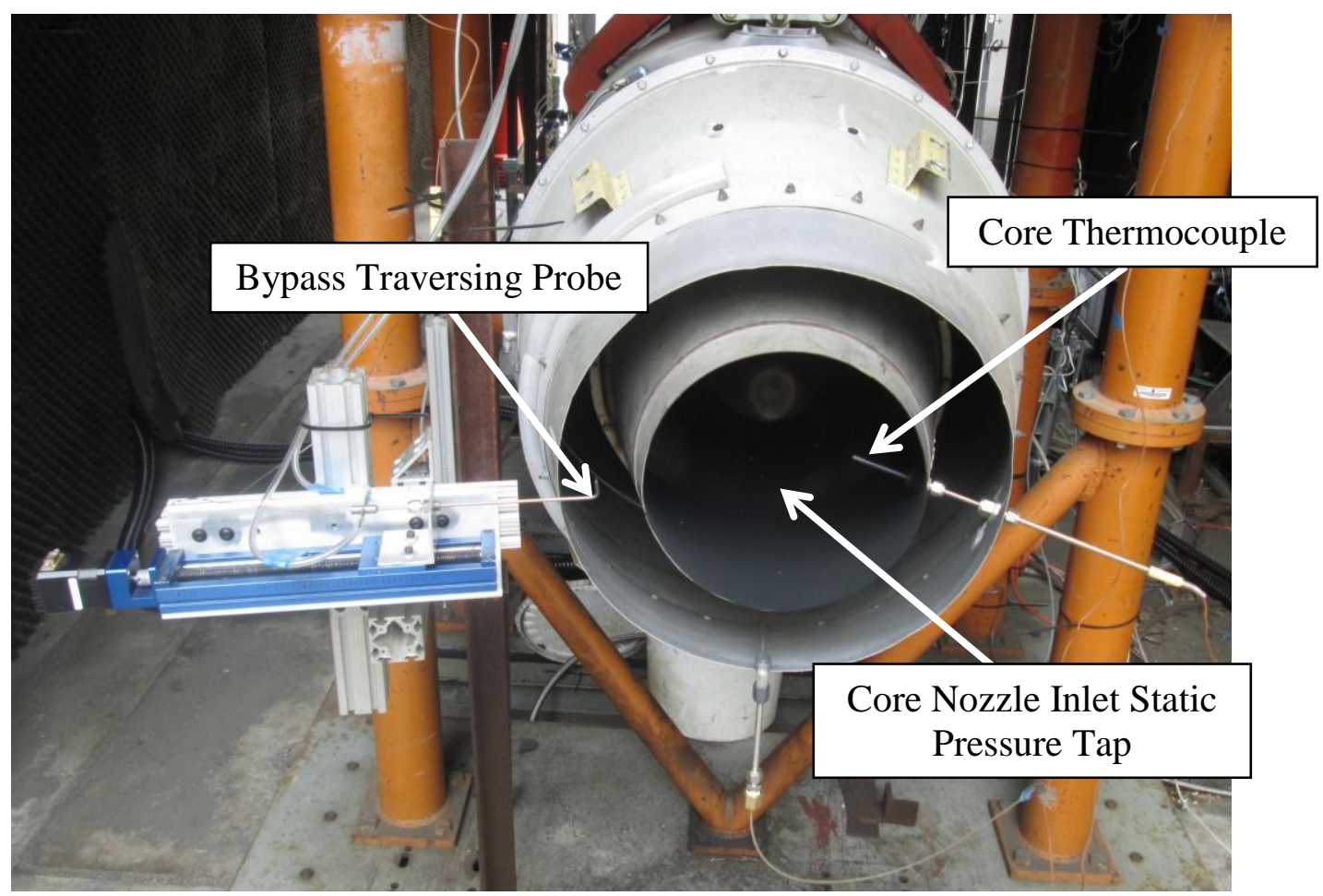

Figure 2.3: Bypass and Core Flow Measurement Probes

The fuel consumption of the engine was monitored and recorded using an Electronics International FT180 Fuel Flow transducer. This transducer was installed in the fuel supply line downstream of the external fuel pump.

Finally, a once-per-revolution optical sensor recorded the fan shaft rotational speed. This optical sensor provided the ability to monitor slight fluctuations in engine rotational speed and account for these changes if they were sufficient to affect the flow profiles measured at the nozzle exit plane.

\subsection{Experimental Setup}

Two separate tests were performed using the modified JT15D-1 engine: a "clean" test and a "distorted" test. The "clean" test was conducted with a backing screen consisting of 0.5 " stainless steel wire mesh with a porosity of 86.1. The "distorted" test was conducted with a distortion screen designed and calibrated to create a profile similar to NASA's Inlet A, a serpentine inlet that ingests $30 \%$ of the boundary layer formed over a typical HWB aircraft. This distortion screen was created by layering fine stainless steel wire mesh with a porosity of 77.5 on top of the backing screen. These fine mesh layers were staggered such that the wires did not overlap, thus creating regions of very low porosity. It should be noted that while each layer of the fine wire mesh has a porosity of 77.5, due to the staggering of the layers of the fine mesh, the 
actual porosity of the distortion screen is unknown. The total pressure profile produced by the screen will be measured to ensure the screen creates the desired profile. The backing and distortion screens used are shown in Figure 2.4.
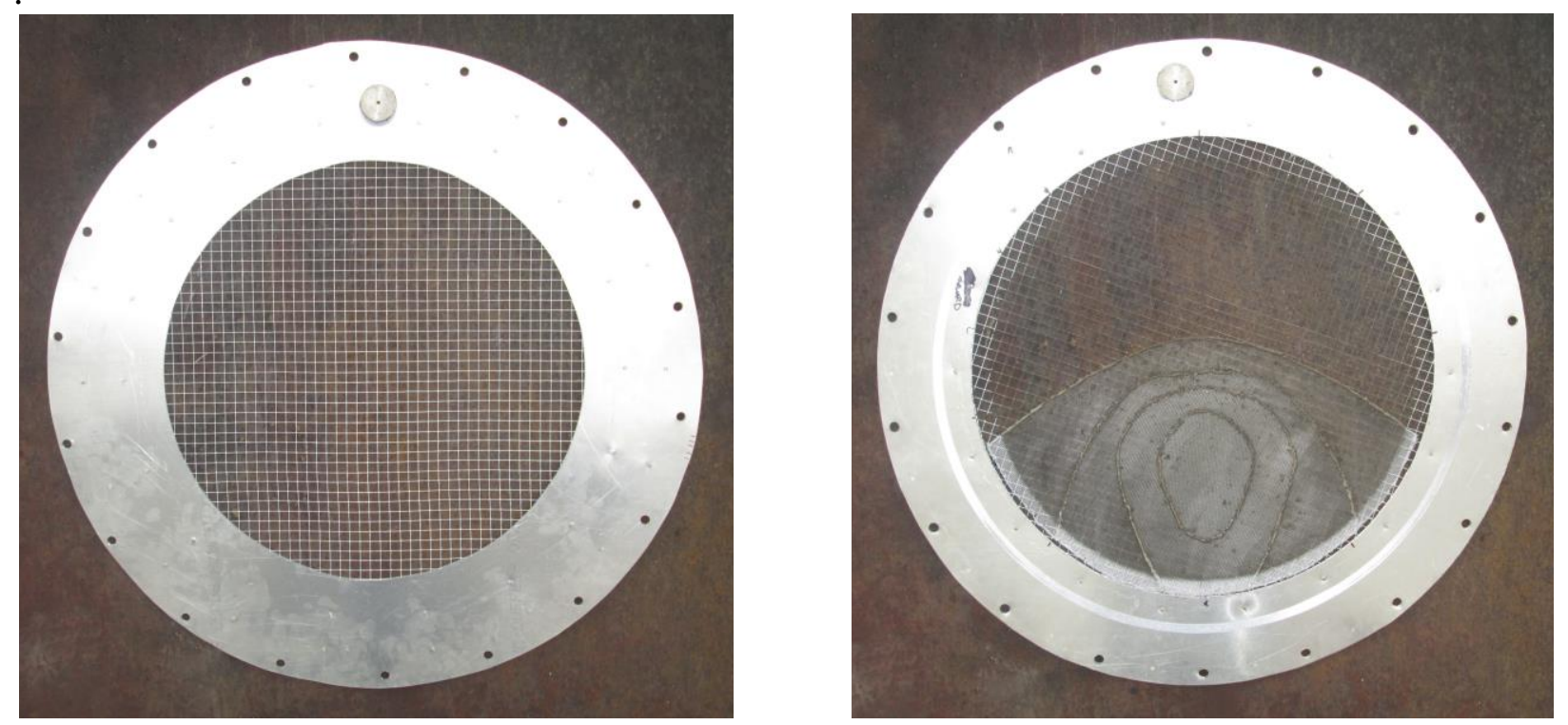

Figure 2.4: Backing Screen (Left) and Distortion Screen (Right)

The data presented in this thesis were collected at a corrected fan speed of $80 \%$. For both the "clean" and "distorted" tests, a probe located at the bypass nozzle exit plane was traversed radially from the inner edge of the bypass nozzle to the outer edge of the core nozzle in 0.125 inch increments. The total length of travel was 2.5 inches. The "clean" test was conducted at one screen position, i.e. the backing screen was not rotated due to its symmetric nature. The "distorted" test was conducted with 24 distortion screen positions, rotating the distortion screen in 15 degree increments.

Pressure measurements from the AIP rake were acquired using Omega PX139 transducers connected to a National Instruments (NI) PXI-6255 multifunction DAQ card. These AIP pressure measurements were sampled at $128 \mathrm{~Hz}$. The Omega PX139 transducers were calibrated by applying known pressures as measured by a Dwyer Series 477A Digital Manometer. Pressure measurements from the bellmouth static taps, bypass traversing probe, and core static tap were collected using a self-calibrating Measurement Specialties NetScanner Model 98RK-1 pressure scanner with a 5 psig module. These pressure measurements were sampled at $4 \mathrm{~Hz}$. The core and bypass temperature measurements were acquired using an NI cDAQ-9172 chassis with an NI 9211 thermocouple module, sampled at $8 \mathrm{~Hz}$. The output from the optical once-per revolution sensor was input to a Monarch Instruments ACT-3X tachometer. In addition to displaying the engine RPM, this tachometer also transforms the input signal into a square wave. The outputs from the Monarch Instruments tachometer and the fuel flow meter were recorded using an NI USB-6259 screw terminal DAQ card. These outputs were sampled at $50 \mathrm{kHz}$. Standard practice and manufacturers recommendations were followed to calibrate all instruments used in this investigation. All data collection and motion controls were integrated using LabVIEW software on a computer. All measurements were acquired for 5 seconds at each screen position and bypass traversing probe radial location. 


\section{Data Analysis Methods}

\subsection{Distortion Characterization}

Due to the numerous possible shapes and magnitudes of inlet flow distortion, it is necessary to develop parameters that describe the distortion. These parameters include average total pressure, minimum total pressure, circumferential distortion extent, circumferential distortion intensity, multiple-per-revolution, and radial distortion intensity. The average total pressures at both the AIP and bypass nozzle exit plane were computed through an arithmetic mean of pressures measured at the mid-radius of five equal area rings. This computation resulted in an areaweighted mean total pressure for both measurement planes. The average total pressure recovery, $\overline{R_{p}}$, is defined in Equations 3.1 and 3.2.

$$
\begin{gathered}
\overline{R_{p}}=\frac{\overline{p_{0}}}{p_{a t m}} \\
\overline{p_{0}}=\frac{1}{n} \sum_{i=1}^{n} p_{0 i}
\end{gathered}
$$

A distortion analysis methodology is described in the Society of Automotive Engineers document ARP $1420^{19}$. Additional distortion indices are defined based on total pressure profiles of individual rings within a duct. Unwrapping this total pressure profile for a single ring allows the computation of circumferential distortion extent, circumferential distortion intensity, multiple-per-revolution, and radial distortion intensity. A sample once-per-revolution ring total pressure profile is shown in Figure $3.1^{20}$.

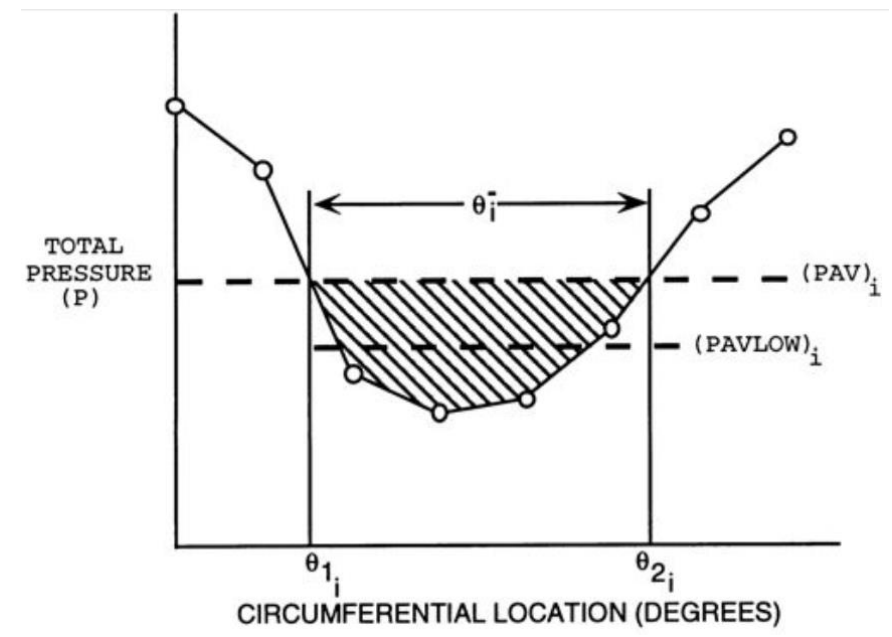

Figure 3.1: Sample Once-Per-Revolution Ring Total Pressure Profile with ARP1420 Distortion Parameters 
The circumferential distortion extent for a given ring, $\theta_{i}^{-}$, is the angular distance in degrees for which the local total pressure falls below the ring average total pressure, as defined in Equation 3.3 , where $i$ corresponds to a single ring.

$$
\theta_{i}^{-}=\theta_{2_{i}}-\theta_{1_{i}}
$$

The circumferential distortion intensity, $\left(\frac{\Delta p_{c}}{p}\right)$, computed using Equations 3.4, 3.5, and 3.6, quantifies the amount of total pressure variation around the ring.

$$
\begin{gathered}
\left(\frac{\Delta p_{c}}{p}\right)_{i}=\left(\frac{P A V-P A V L O W}{P A V}\right)_{i} \\
P A V_{i}=\frac{1}{360} \int_{0}^{360} P(\theta)_{i} d \theta \\
P A V L O W_{i}=\frac{1}{\theta_{i}^{-}} \int_{\theta_{1 i}}^{\theta_{2 i}} P(\theta)_{i} d \theta
\end{gathered}
$$

The multiple-per-revolution parameter describes how many low total pressure regions exist around a given ring. A low total pressure region occurs when the local total pressure, $P(\theta)_{i}$, lies below the ring average total pressure, $P A V_{i}$. If two low total pressure regions occur within 25 degrees of each other, it is treated as a single once-per-revolution low total pressure region. If two low total pressure regions are separated by more than 25 degrees, the circumferential distortion intensity is taken to be the maximum of the value described in Equation 3.7.

$$
\left(\frac{\Delta p_{c}}{p}\right)_{i} \theta_{i}^{-}
$$

In this scenario, the circumferential distortion extent is taken to be the circumferential distortion extent of the region that makes the value given in Equation 3.7 a maximum.

The radial distortion intensity, $\left(\frac{\Delta p_{r}}{p}\right)_{i}$, computed using Equations 3.8 and 3.9, quantifies the amount of radial total pressure variation present in the distortion. The radial distortion intensity is the difference between the ring average pressure, $P A V_{i}$, and the face average pressure, $P F A V$, for each ring.

$$
\begin{gathered}
\left(\frac{\Delta p_{r}}{p}\right)_{i}=\frac{P F A V-P A V_{i}}{P F A V} \\
P F A V=\frac{1}{N} \sum_{i=1}^{N} P A V_{i}
\end{gathered}
$$


SAE ARP 1420 recommends calculating the distortion indices described using a rake of probes with each probe positioned in the center of equal area rings. The AIP rake described in Section 2.3 was designed according to this recommendation. Similarly, five radii traversed by the bypass traversing probe were selected such that the bypass flow measurements were split into five equal area rings. This allows for the flow profiles at the AIP to be compared to the flow profiles at the bypass nozzle exit. However, the flow splits into core and bypass flows downstream of the AIP. This prohibits the comparison of the five AIP rings to the five bypass rings, as not all of the flow measured by the five AIP rings travels through into the bypass. To correct this issue, the flow at the AIP was split into two regions: a bypass flow region and core flow region. Due to flow measurement limitations, the mass flow distribution at the AIP could not be calculated. Thus, it was estimated that the area ratio between the bypass and core flow regions at the AIP would be equal to the calculated bypass ratio (see Section 3.2 for calculation). Once the AIP had been split into bypass and core flow regions, the bypass flow region was split into five equal area rings. The AIP measurements lying within this region were linearly interpolated or extrapolated to five radii located at the center of each of the five bypass flow region rings. This allowed for the direct comparison of flow parameters at the AIP to those at the bypass nozzle exit plane.

The values of the various distortion characterization parameters were used to compare the "distorted" test flow profile to that produced by NASA's Inlet A, thus verifying that the distortion generated by the distortion screen was comparable to the distortion produced by Inlet A. The distortion indices were also used to characterize the difference between the "clean" test flow profile and the "distorted" test flow profile.

\subsection{Engine Performance Calculations}

In addition to the distortion characterization parameters described above, many other performance related parameters were used to quantify the performance change of the modified JT15D-1 engine in the presence of distortion typical of BLI embedded engine systems. These parameters include corrected stream thrust, corrected thrust-specific fuel consumption (TSFC), and fuel consumption.

In the absence of the instrumentation necessary to measure the thrust of the engine directly, the stream thrust produced by the engine can be calculated using flow measurements at the inlet and exit of the engine. This stream thrust is essentially the force required to accelerate the stationary free stream air to the velocity measured at the nozzle exit plane as derived from the conservation of momentum equation. The control volume and equations used for the stream thrust calculation are shown in Figure 3.2 and Equations 3.10, 3.11, and 3.12. Referenced to $u_{\infty}$, the inlet stream thrust, $\mathrm{T}_{\infty}$, is taken to be zero. 


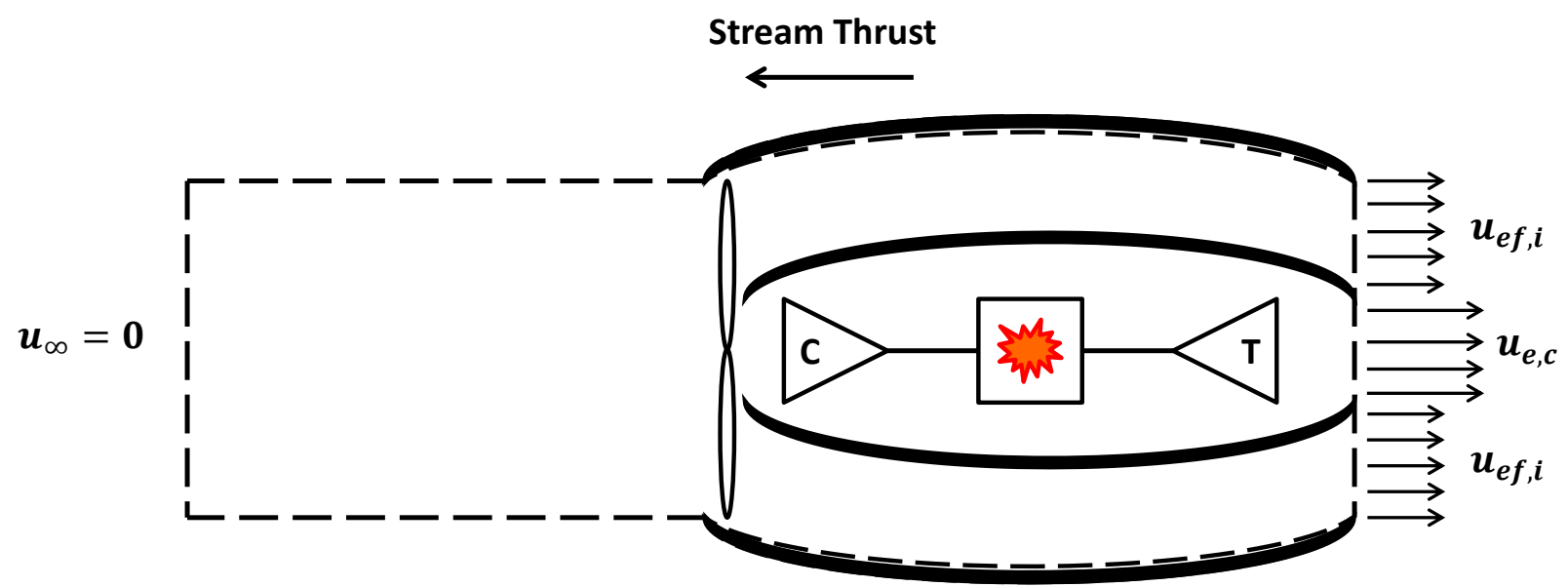

Figure 3.2: Stream Thrust Calculation Control Volume

$$
\begin{gathered}
\mathrm{T}_{\mathrm{s}}-\mathrm{T}_{\infty}=\mathrm{T}_{s c}+\sum_{i=1}^{n} \mathrm{~T}_{s b, i} \\
\mathrm{~T}_{s b, i}=\dot{m}_{b, i} u_{e f, i} \\
\mathrm{~T}_{s c}=\dot{m}_{c} u_{e c}
\end{gathered}
$$

Once the total engine stream thrust had been calculated, it was corrected for ambient conditions using Equations 3.13 and 3.14,

$$
\mathrm{T}_{\mathrm{s}, \mathrm{cor}}=\frac{\mathrm{T}_{\mathrm{s}}}{\delta}
$$

where

$$
\delta=\frac{p}{p_{\text {ref }}}
$$

The reference pressure, $p_{\text {ref }}$, was $101,325 \mathrm{~Pa}$. Next, corrected thrust-specific fuel consumption was calculated using Equations 3.15 and 3.16,

$$
T S F C_{c o r}=\frac{\dot{m}_{f}}{\mathrm{~T}_{\mathrm{s}} \sqrt{\Theta}}
$$

where

$$
\Theta=\frac{T}{T_{\text {ref }}}
$$

The reference temperature, $T_{\text {ref }}$, was $288.15 \mathrm{~K}$. The final performance parameter calculated was the bypass ratio, $\beta$, given in Equation 3.17.

$$
\beta=\frac{\dot{m}_{b}}{\dot{m}_{c}}
$$


In order to calculate the performance parameters described above, several calculations were performed to determine the mass flows and nozzle exit velocities for both core and bypass flows. First, the bypass mass flow for each stream tube, $\dot{m}_{b, i}$, was calculated using measured bypass exit total and static pressure values and Equation 3.18 to find the Mach number at the bypass nozzle exit plane for each stream tube. This bypass exit Mach number was then inserted into Equation 3.19, along with measured bypass nozzle exit total temperature values.

$$
\begin{gathered}
\frac{p_{0 i}}{p_{i}}=\left(1+\frac{\gamma-1}{2} M_{i}^{2}\right)^{\frac{\gamma}{\gamma-1}} \\
\dot{m}_{b, i}=A_{i} M_{i} \frac{p_{0 i} \sqrt{\gamma}}{\sqrt{R T_{0 i}}}\left(\frac{1}{1+\frac{\gamma-1}{2} M_{i}^{2}}\right)^{\frac{\gamma+1}{2(\gamma-1)}}
\end{gathered}
$$

The Mach number for each stream tube, calculated using Equation 3.18, was also used to calculate the exit velocity for each stream tube, as shown in Equation 3.20.

$$
u_{e f, i}=M_{i} \sqrt{\frac{\gamma R T_{0 i}}{1+\frac{\gamma-1}{2} M_{i}^{2}}}
$$

Next, the core mass flow, $\dot{m}_{c}$, was calculated using the fuel mass flow measured by the FT180, the bellmouth mass flow measured using the calibrated bellmouth inlet, and the total bypass mass flow calculated using Equation 3.21. The core mass flow equation is shown as Equation 3.22 .

$$
\begin{gathered}
\dot{m}_{b}=\sum_{i=1}^{n} \dot{m}_{b, i} \\
\dot{m}_{c}=\dot{m}_{a, \text { bell }}-\dot{m}_{b}+\dot{m}_{f}
\end{gathered}
$$

It should be noted that the core mass flow calculated using Equation 3.22 includes both the core air mass flow and fuel mass flow introduced in the combustor. Once the core mass flow was known, the core exit velocity could be calculated using the measured core static pressure and total temperature along with a series of equations. Derivation of the following core flow parameter equations can be found in Appendix A. First, Equation 3.23 was iteratively solved to find the total pressure at the core nozzle inlet, $p_{01, c}$.

$$
\dot{m}_{c}=\frac{A_{1, c} p_{1, c} \sqrt{\frac{2 \gamma_{c}}{\gamma_{c}-1}\left(\left(\frac{p_{01, c}}{p_{1, c}}\right)^{\frac{\gamma_{c}-1}{\gamma_{c}}}-1\right)}}{\sqrt{R T_{0, c}\left(\frac{p_{1, c}}{p_{01, c}}\right)^{\frac{\gamma_{c}-1}{\gamma_{c}}}}}
$$


Once the core nozzle inlet total pressure was known, the core nozzle pressure ratio, $\lambda_{c}$, was determined by iteratively solving Equation 3.24.

$$
\begin{aligned}
& p_{1, \mathrm{c}}^{2} A_{1, \mathrm{c}}^{2}\left(\left(\frac{p_{01, c}}{p_{1, c}}\right)^{\frac{2\left(\gamma_{c}-1\right)}{\gamma_{c}}}-\left(\frac{p_{01, c}}{p_{1, c}}\right)^{\frac{\gamma_{c}-1}{\gamma_{c}}}\right)= \\
& p_{2, \mathrm{c}}^{2} A_{2, \mathrm{c}}^{2}\left(\left(\frac{\lambda_{c} p_{01, c}}{p_{2, c}}\right)^{\frac{2\left(\gamma_{c}-1\right)}{\gamma_{c}}}-\left(\frac{\lambda_{c} p_{01, c}}{p_{2, c}}\right)^{\frac{\gamma_{c}-1}{\gamma_{c}}}\right)
\end{aligned}
$$

Equation 3.25 was then used to calculate the total pressure at the core nozzle exit plane.

$$
p_{02, c}=\lambda_{c} p_{01, c}
$$

Next, because the core nozzle was not choked, it was assumed that the static pressure at the core nozzle exit plane was the ambient pressure. This allowed for the calculation of the core nozzle exit Mach number using Equation 3.26.

$$
M_{2, c}=\sqrt{\frac{2}{\gamma-1}\left(\left(\frac{p_{02}}{p_{2}}\right)^{\frac{\gamma_{c}-1}{\gamma_{c}}}-1\right)}
$$

Finally, the core exit velocity could be calculated using Equation 3.27.

$$
u_{e c}=M_{2, c} \sqrt{\frac{\gamma_{c} R T_{0, c}}{1+\frac{\gamma_{c}-1}{2} M_{2, c}^{2}}}
$$

\subsection{Flow Loss Calculations}

Once the engine performance values had been calculated for both the clean and distorted tests, it was desired to track the flow losses through the engine. Due to the placement of instrumentation, it was only possible to calculate flow losses between ambient conditions, the aerodynamic interface plane (AIP), and the bypass nozzle exit. The flow stations used for the flow loss calculations are shown in Figure 3.3. Station 0 represents ambient conditions, Station 1 represents the AIP, and Station 2 represents the bypass nozzle exit plane. The flow losses were calculated along the flow path shown in red in Figure 3.3, hereafter referred to as the fan flow path. 


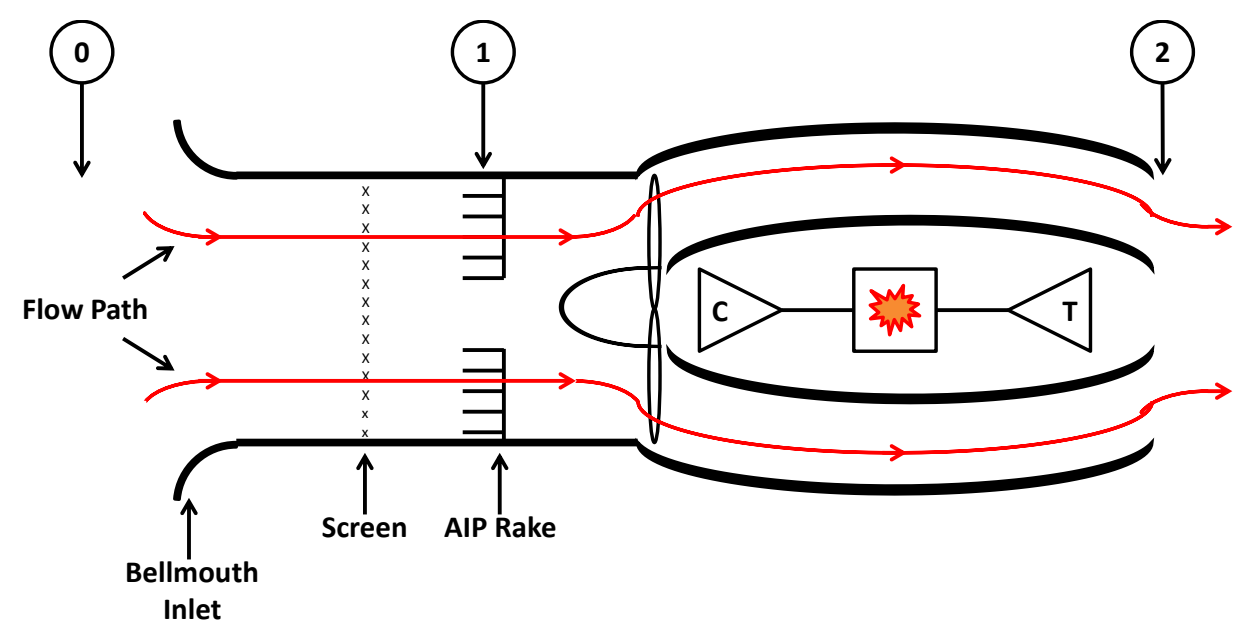

Figure 3.3: Flow Stations for Flow Loss Calculations

The main parameter used to describe flow losses in this analysis was the entropy generation between two stations. Assuming constant specific heat capacity at the average temperature between the two stations, Equation 3.28 was used to calculate the entropy generation within a streamtube between measurement point $i$ at Station $j$ and measurement point $i$ at Station $k$. An analsyis of the minimal effect of the constant specific heat capacity assumption can be found in Appendix B.

$$
\Delta s_{j k, i}=c_{p} \ln \left(\frac{T_{0 i, k}}{T_{0 i, j}}\right)-R \ln \left(\frac{p_{0 i, k}}{p_{0 i, j}}\right)
$$

Using Equation 3.28, the overall entropy generation for the entire "red line" flow path was calculated by mass averaging the entropy generated between Stations 0 and 2, as shown in Equation 3.29.

$$
\Delta \bar{s}_{02}=\frac{\sum_{i=1}^{n} \dot{m}_{\mathrm{b}, \mathrm{i}} \Delta s_{02, i}}{\dot{m}_{b}}
$$

This overall entropy generation was compared for both clean and distorted tests. As described in Section 3.1, the mass flow distribution at the AIP could not be calculated. Thus, a mass averaged entropy generation at the AIP was not able to be calculated. An area averaged entropy generation was not considered appropriate for use, so plots of the entropy generated between Stations 0 and 1 for each AIP rake measurement were created, and calculated using Equation 3.28. Similar plots were created for the entropy generated between Stations 0 and 2. By comparing these two plots, the entropy produced by the screen was related to the entropy produced through the entire fan flow path. By the First Law of Thermodynamics, the total temperature must remain constant across the distortion screen, allowing for Equation 3.28 to be used with only total pressure measurements at the AIP (see Appendix C for proof). It should be noted that the flow parameters at Station 1 were determined using only the assumed bypass flow region of the AIP as described in Section 3.1. 
In addition to the entropy generated between Stations 0 and 2, a fan flow path efficiency, $\eta_{f f p}$, was calculated using Equation 3.30.

$$
\eta_{f f p}=\frac{\left(\left(\frac{p_{02}}{p_{a t m}}\right)^{\frac{\gamma-1}{\gamma}}-1\right)}{\left(\frac{T_{02}}{T_{a t m}}-1\right)}
$$

The entropy generation and fan flow path efficiency values were compared for both clean and distorted tests to help determine the magnitude and location of the flow losses due to the embedded BLI-type distortion. 


\section{Results and Discussion}

\subsection{Experimental Conditions}

Due to the complexity of the experimental setup and the relatively large time requirements for the distorted test, the clean and distorted tests were performed on two separate days. For each run, ambient conditions and nominal engine performance parameters were recorded. Table 4.1 presents a summary of these values.

Table 4.1: Comparison of Conditions for Clean and Distorted Engine Tests

\begin{tabular}{|c|c|c|}
\hline Parameter & Clean & Distorted \\
\hline Ambient Pressure [psi] & 14.92 & 14.81 \\
\hline Ambient Temperature [ $\left.{ }^{\circ} \mathrm{C}\right]$ & 13.9 & 16.0 \\
\hline Relative Humidity [\%] & 77 & 48 \\
\hline Corrected Fan Speed [\%] & 79.64 & 79.07 \\
\hline Corrected Core Speed [\%] & 85 & 85 \\
\hline ITT [ ${ }^{\circ} \mathrm{C}$ ] & 580 & 581 \\
\hline
\end{tabular}

As mentioned in Section 2.4, it was desired for tests to be run at a corrected fan speed of $80 \%$. As shown in Table 4.1, the actual engine speed fell just below the desired $80 \%$ for both tests, with the distorted test being $0.57 \%$ slower than the clean test. According to a study by Alhamaly ${ }^{23}$, this difference results in a less than $1 \%$ change in fan performance, so it was concluded that the results from the two tests could be compared accurately.

\subsection{Characterization of Distortion}

In order to compare the clean and distorted performance, it is necessary to characterize the amount of distortion present for each test. Figure 4.1 shows the contours of total pressure recovery at the AIP for both the clean and distorted tests. The total pressure recovery is defined in Equation 4.1. 


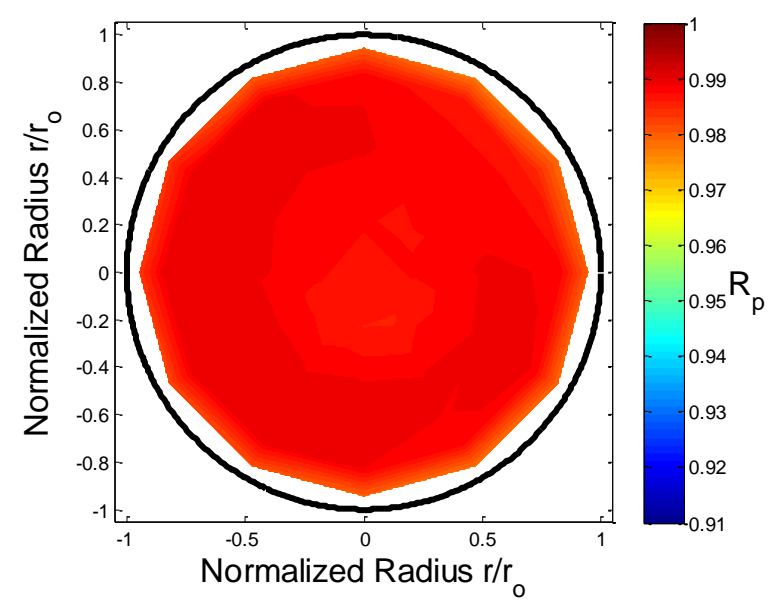

(a)

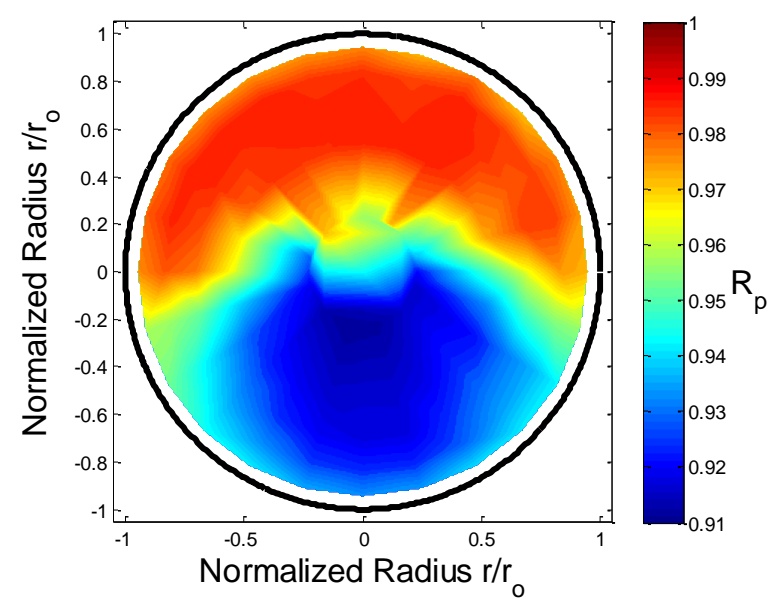

(b)

Figure 4.1: Contours of Total Pressure Recovery at AIP for (a) Clean Test and (b) Distorted Test

$$
R_{p}=\frac{p_{0 i}}{p_{a t m}}
$$

Comparing the two contour plots in Figure 4.1, it can be seen that the distortion screen created a large region of low total pressure flow. The total pressure recovery for the clean test ranged from 0.977 to 0.990 with an average of 0.987 , while the total pressure recovery for the distorted test ranged from 0.912 to 0.987 with an average of 0.954 . It should be noted that the small amount of non-uniformity in the clean total pressure recovery contour is likely due to inlet duct non-uniformities.

In addition to contour plots of the AIP total pressure measurements, the total pressure measurements around each of the five rings of the AIP rake can be plotted as a function of circumferential angle, $\theta$. These plots will be referred to as "unwrap plots." Figure 4.2 shows these unwrap plots. Ring 1 is closest to the center axis of the engine, with each ring expanding radially outward. $\theta=0$ corresponds to top dead center of the contours shown in Figure 4.1 with $\theta$ increasing in the clockwise direction when looking at the engine face. 

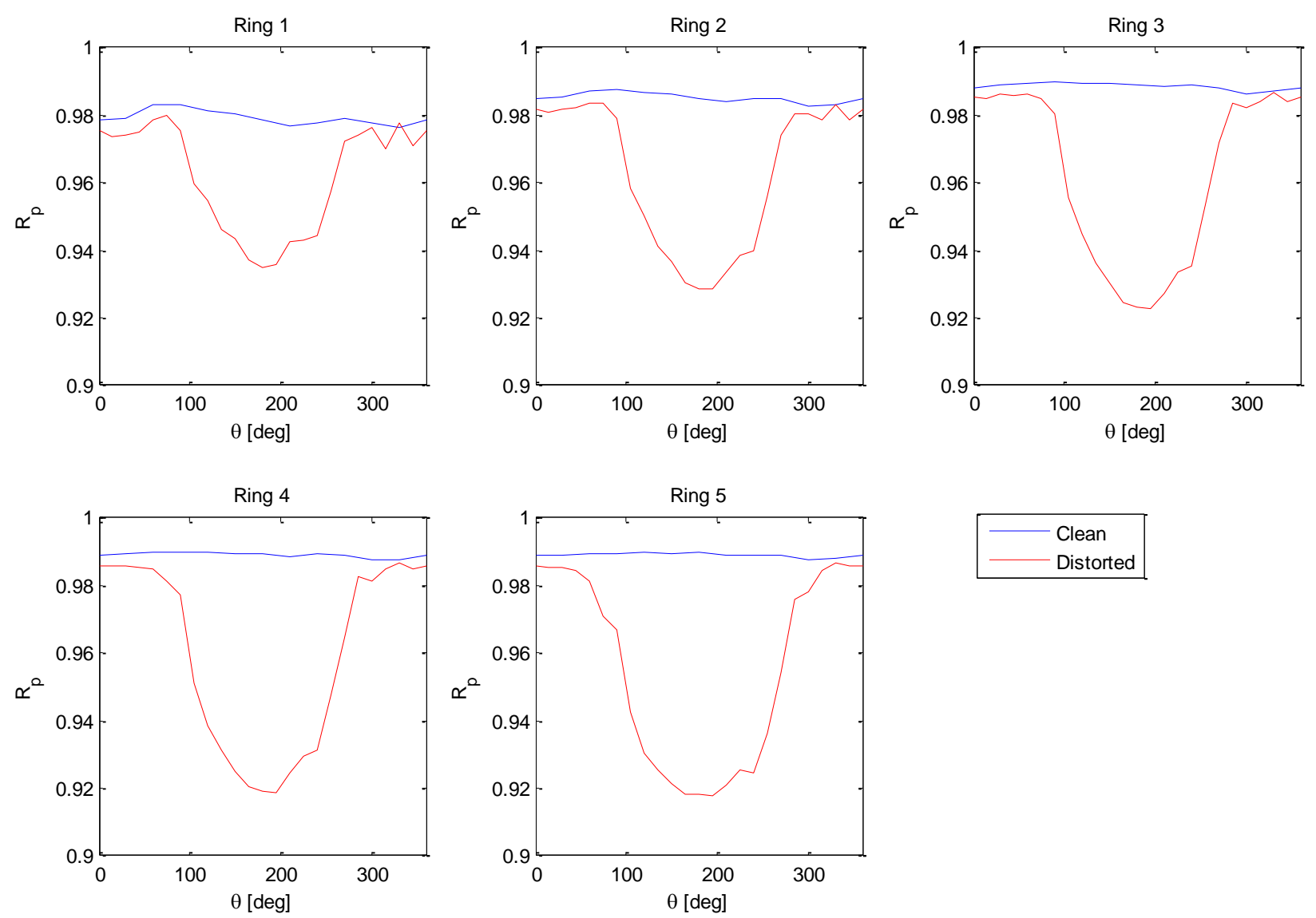

Clean

Distorted

Figure 4.2: Unwrap Plots of Clean and Distorted Total Pressure Recovery

As shown in Figure 4.2, the distorted total pressure recovery decreases from $\theta=0^{\circ}$ to $\theta=180^{\circ}$, then increases from $\theta=180^{\circ}$ to $\theta=360^{\circ}$ for every ring, while the clean total pressure recovery remains relatively constant. Also, the minimum total pressure recovery decreases towards the outer radii. These trends further emphasize the trends shown in Figure 4.1.

Additionally, the distorted AIP total pressure profile can be characterized using the distortion indices described in Section 3.1. The distortion screen was designed to mimic the distortion produced by NASA's Inlet A. Thus, the distortion indices for Inlet A, as computed by $\mathrm{Ferrar}^{20}$, can be compared to the distortion indices for the distorted test. Plots of circumferential intensity can be found in Figure 4.3, plots of radial intensity can be found in Figure 4.4, and plots of extent can be found in Figure 4.5. 


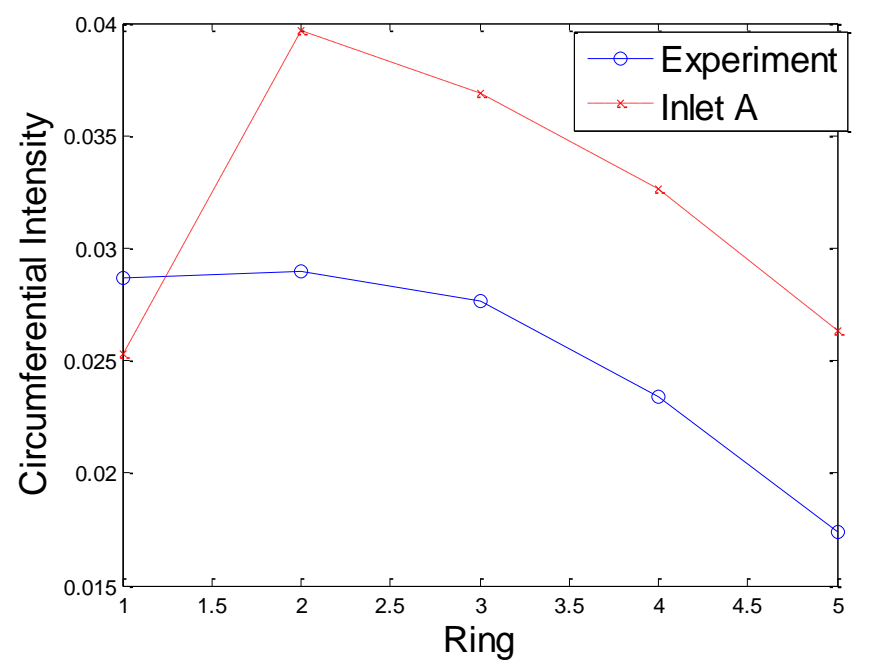

Figure 4.3: Circumferential Intensity at AIP for Distorted Test and Inlet A

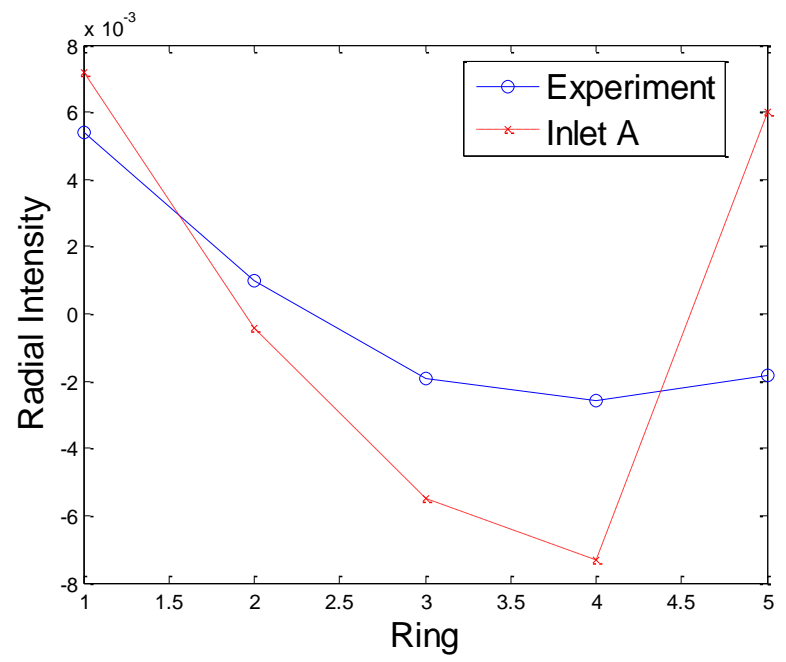

Figure 4.4: Radial Intensity at AIP for Distorted Test and Inlet A

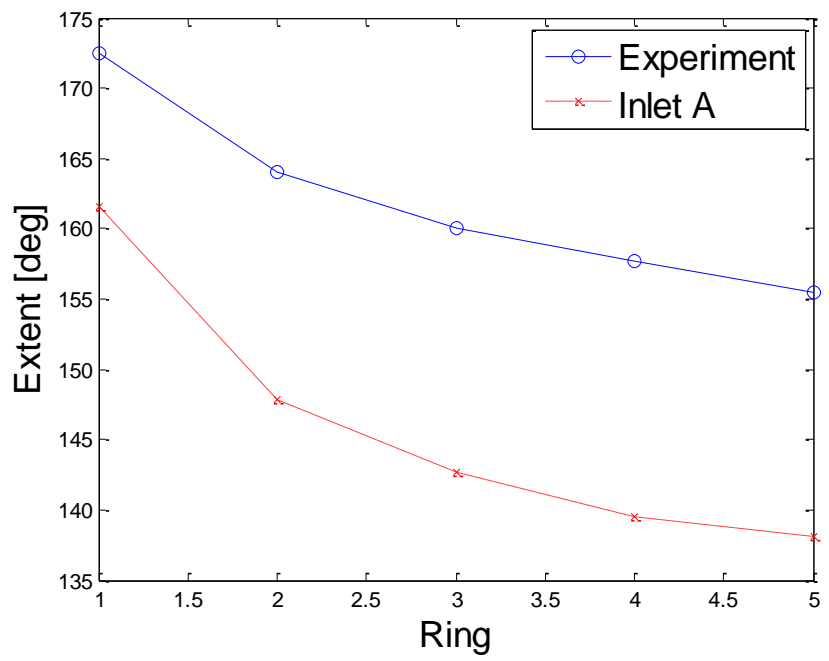

Figure 4.5: Extent at AIP for Distorted Test and Inlet A 
As shown in Figure 4.3, Figure 4.4, and Figure 4.5, the distortion indices produced for the distorted test are comparable to those produced by Inlet A. The circumferential intensity produced by the distortion screen, shown in Figure 4.3, is not quite as high as that produced by Inlet A, however, the trends are very similar, especially toward the outer radii. The radial intensity curves, shown in Figure 4.4, are also very comparable with similar trends and magnitude. The extent curves, shown in Figure 4.5, also have similar shapes, while the extent produced by the distortion screen is greater than that of Inlet A. Thus, while the circumferential intensity of the distortion screen was lower than that of Inlet A, and the extent produced by the distortion screen was larger than that of Inlet A, all distortion indices were still comparable in trend, and magnitude. This lead to the conclusion that the distortion screen produced a distortion similar to that of a typical boundary layer ingesting embedded engine inlet.

\subsection{Performance Comparison}

After verifying that the distortion screen produced the desired amount of distortion, the performance of the engine with and without the distortion screen was analyzed. The results of the performance calculations described in Section 3.2 are summarized in Table 4.2. A description of the uncertainty analysis performed can be found in Appendix B.

Table 4.2: Comparison of Performance Parameters for Clean and Distorted Tests

\begin{tabular}{|c|c|c|c|c|}
\hline Parameter & Clean & Distorted & \% Difference & Uncertainty \\
\hline Corrected Stream Thrust & $1058.44 \mathrm{lbf}$ & $894.26 \mathrm{lbf}$ & $-15.51 \%$ & $\pm 0.92 \%$ \\
\hline Corrected TSFC & $0.7005 \mathrm{lbm} / \mathrm{hr} / \mathrm{lbf}$ & $0.7975 \mathrm{lbm} / \mathrm{hr} / \mathrm{lbf}$ & $+13.85 \%$ & $\pm 1.94 \%$ \\
\hline Fuel Consumption & $109.82 \mathrm{GPH}$ & $104.83 \mathrm{GPH}$ & $-4.54 \%$ & $\pm 1.03 \%$ \\
\hline
\end{tabular}

As shown in Table 4.2, the presence of the distortion screen created a significant change in the modified JT15D-1 engine's performance. The loss in stream thrust and increase in TSFC were expected according to the results of the studies presented in Section 1.2.

While the stream thrust loss and TSFC increase for a given engine speed is harmful from an engine performance perspective, this stream thrust loss could prove to be beneficial in the right context. According to a design study by Liebeck, HWB aircraft could require 19\% - 27\% less thrust than comparably sized tube and wing aircraft ${ }^{21}$. Thus, depending on the magnitude of the thrust requirements for a HWB aircraft, the thrust loss due to BLI may not be as detrimental. The detrimental impact of BLI-type distortion is further reduced when the raw fuel consumption is considered. As Table 4.2 shows, even though the corrected TSFC increased substantially, the raw fuel consumption actually decreased by $4.54 \%$ in the presence of distortion. Consequently, if the reduction in thrust generated by the engine is within the reduction of required thrust for the aircraft, BLI engines could actually burn less fuel.

It should be noted that while this discussion suggests potential benefits due to reduced engine performance in the presence of BLI distortion, these results only pertain to one particular engine at one particular speed. More experiments at multiple engine speeds with various distortions on modern engines would be required to make a more definitive assessment. 


\subsection{Performance Loss Analysis}

While quantifying and analyzing the performance changes in the modified JT15D-1 engine in the presence of BLI-type distortion is interesting, this engine would never be used in a BLI application. Thus, it is far more practical for modern aviation research to investigate the causes of the reduced performance of this engine in an attempt to identify areas of high loss that could be further investigated in future research.

\subsubsection{Thrust Loss Mechanisms}

By the conservation of momentum equation (see Equations $3.10-3.12$ ), reductions in air mass flow rate, bypass nozzle exit velocity, and/or core nozzle exit velocity result in a reduction in stream thrust. The blockage created by the distortion screen resulted in a $8.59 \%$ reduction in corrected air mass flow at the bellmouth inlet. The core nozzle average exit velocity was reduced by $2.5 \%$ and the bypass nozzle average exit velocity was reduced by $9.85 \%$ in the presence of BLI distortion. These reductions in air mass flow rate and nozzle exit velocities are the primary contributors to the reduction in stream thrust in the presence of BLI distortion.

It is also interesting to analyze the change in bypass ratio due to the BLI distortion screen. The bypass ratio for the clean inlet test was calculated to be 3.06, while the bypass ratio for the distorted test was calculated to be 2.85 , a $6.84 \%$ reduction. In turbofan engines, the majority of the thrust produced comes from the bypass flow. A reduction in bypass ratio, therefore, means a reduction in the amount of flow through the bypass, which would create a decrease in thrust, or stream thrust in this case. It is theorized that the reduction in bypass ratio is a direct result of flow redistribution downstream of the distortion screen. The localized low total pressure regions result in pressure gradients downstream of the distortion screen. In these areas, flow in regions of high pressure will tend to fill in the low pressure regions. This flow redistribution causes some of the flow that would have entered the bypass in the absence of the distortion screen to now enter the core. This would result in a reduction in the amount of air flowing through the bypass, which would also result in a decrease in stream thrust.

\subsubsection{Nozzle Flow Profiles}

After determining that reductions in air mass flow and nozzle exit velocities were the major contributors to the decrease in performance, it was desired that the sources of these reductions be determined. As mentioned above, the reduction in air mass flow rate is primarily due to the flow area blockage created by the distortion screen. However, the source of the reduction in nozzle exit velocities was not as obvious. Because the reduction in the bypass nozzle exit velocity was much greater than that of the core nozzle exit velocity, the bypass flow was analyzed in greater detail.

First, the flow measurements made by the bypass traversing probe for both clean and distorted tests were analyzed. The total pressure ratio, calculated using the same method as shown in 
Equation 4.1, was used to create the contour plots shown in Figure 4.6. The outer black circle represents the location of the bypass nozzle wall, while the inner black circle represents the location of the core nozzle wall.

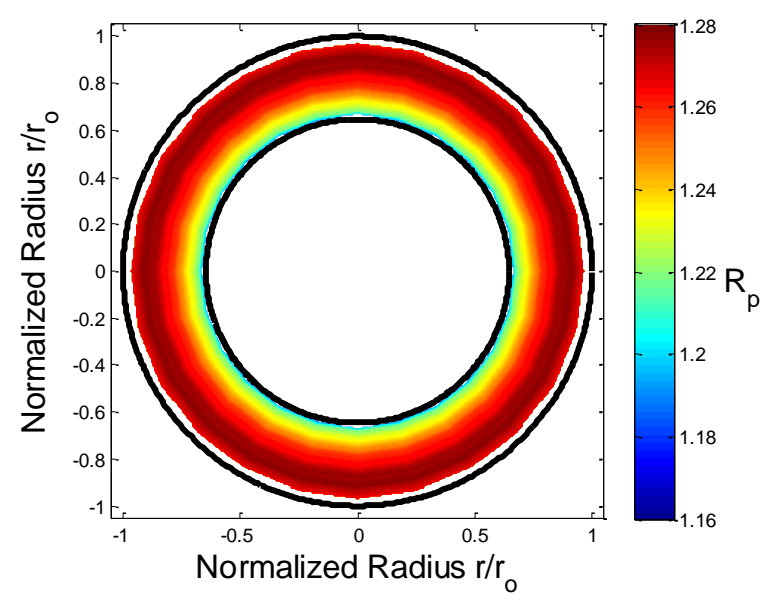

(a)

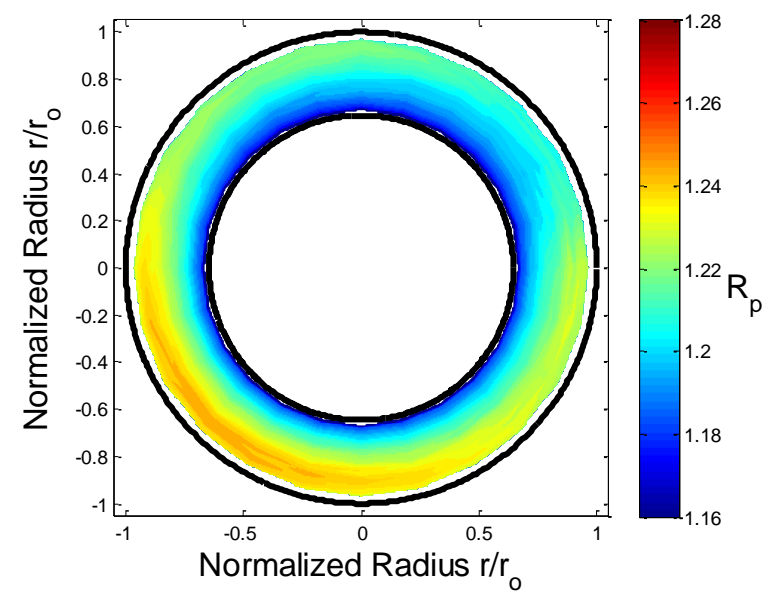

(b)

Figure 4.6: Contours of Total Pressure Ratio at Bypass Nozzle Exit Plane for (a) Clean and (b) Distorted Tests

As shown in Figure 4.6, the total pressure ratio at the nozzle exit plane is significantly lower in the presence of the BLI distortion. The clean test total pressure ratio ranged from 1.189 to 1.279 with an average of 1.255, while the distorted total pressure ratio ranged from 1.112 to 1.246 with an average of 1.208. In addition to the large difference in magnitude of the total pressure ratio between the clean and distorted tests, the difference in shape of the total pressure ratio contours is also interesting. Figure 4.6 (b) shows a region of higher total pressure ratio around the 7 o'clock position and a region of lower total pressure ratio around the 1 o'clock position. This asymmetry is due to the distortion produced by the distortion screen. The off-center shift in the high and low total pressure recovery regions is due to the rotation of the fan. As the distortion is transferred through the fan, the rotational velocity of the fan is also transferred to the flow, causing the distortion to shift circumferentially, resulting in the off-center shift in the total pressure recovery profile shown in Figure 4.6.

Figure 4.7 shows contours of normalized static pressure at the bypass nozzle exit plane for both clean and distorted tests. The normalized static pressure, $p_{\text {norm }}$, is defined as the ratio of the measured local static pressure to ambient pressure. 


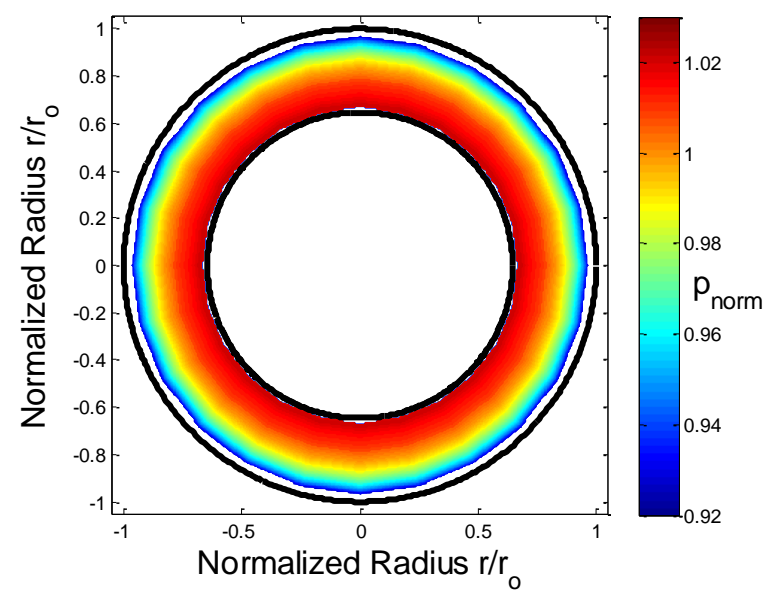

(a)

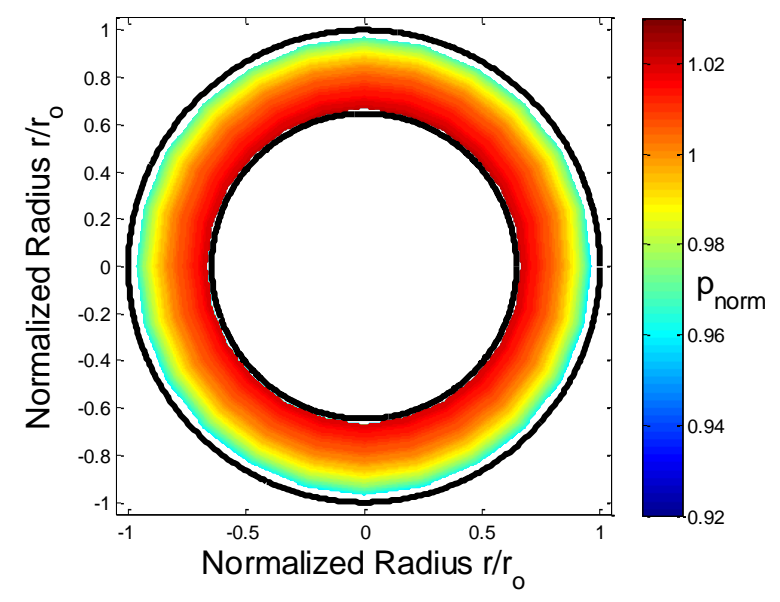

(b)

Figure 4.7: Contours of Normalized Static Pressure at Bypass Nozzle Exit Plane for (a) Clean and (b) Distorted Tests

As shown in Figure 4.7, the static pressure profile is relatively unaffected by the presence of BLI distortion. The static pressure does not decrease so rapidly near the bypass wall for the distorted test, but both profiles are still very similar. The normalized static pressure for the clean test varied from 0.928 to 1.023 with an average of 0.994 . The normalized static pressure for the distorted test varied from 0.956 to 1.024 with an average of 1.000. These values were expected as the bypass nozzle is not choked, meaning the static pressure should approach atmospheric pressure at the bypass nozzle exit. It is also important to note the lack of circumferential variation in the distorted normalized static pressure contour. In contrast to the distorted total pressure ratio contour shown in Figure 4.6 (b), the distorted normalized static pressure contour is fairly uniform around the entire annulus. This indicates that the non-uniformities in the distorted total pressure ratio are related to velocity variation, not static pressure variation.

The final bypass nozzle contour is that of the normalized total temperature, shown in Figure 4.8. The normalized total temperature, $T_{0, n o r m}$, is defined as the ratio of the measured local total temperature to the ambient temperature.

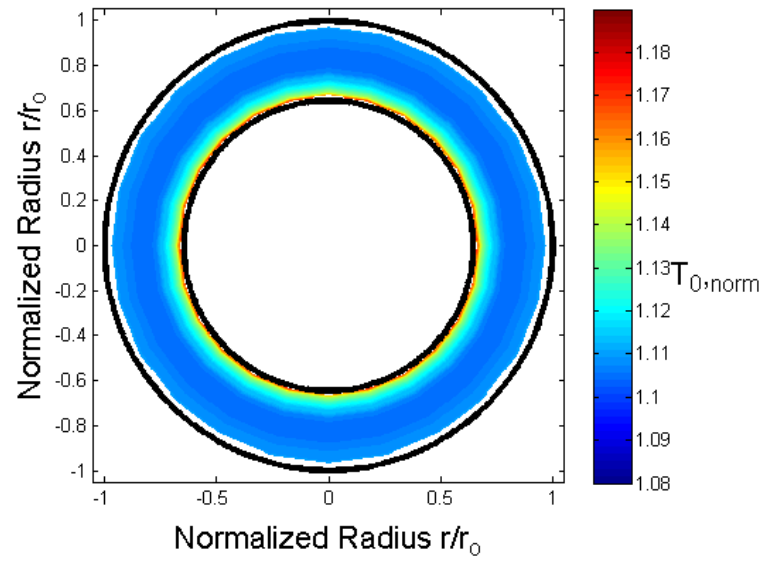

(a)

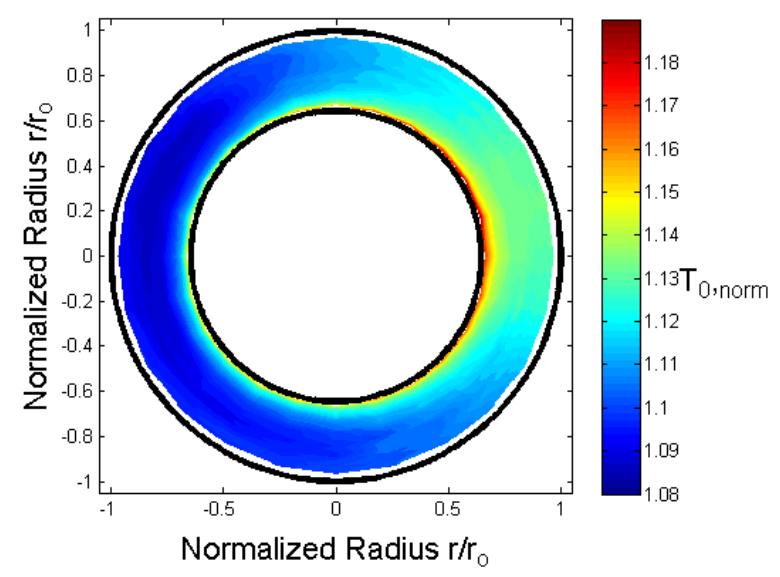

(b)

Figure 4.8: Contours of Normalized Total Temperature at Bypass Nozzle Exit Plane for

(a) Clean and (b) Distorted Tests 
As shown in Figure 4.8, the presence of the BLI distortion affects the normalized total temperature at the bypass nozzle exit plane. For the clean test, the normalized total temperature varied from 1.105 to 1.174 , with an average of 1.115 . For the distorted test, the normalized total temperature varied from 1.086 to 1.192 with an average of 1.114 . Similar to the total pressure recovery contour for the distorted test shown in Figure 4.6 (b), the normalized total temperature for the distorted test has a region of lower normalized total temperature and a region of higher normalized total temperature. The cause of these regions of lower and higher normalized total temperature is likely the same as for the localized lower and higher regions of total pressure recovery shown in Figure 4.6. The increase in work done by the fan in low total pressure regions creates a region of higher total temperature, as well. It is interesting to note, however, that the location of the lower and higher normalized total temperature regions is different from that of the lower and higher total pressure ratio regions. The higher and lower normalized total temperature regions are centered around the 3 o'clock and 9 o'clock positions, respectively, while the higher and lower total pressure ratio regions are centered around the 7 o'clock and 1 o'clock positions, respectively. The phase shift between the normalized total temperature and the total pressure ratio is consistent with the results of simulations conducted by Yao, et al. ${ }^{24,25}$ on the transfer of total pressure distortion through a fan.

Based on the contour plots shown in Figure 4.6, Figure 4.7, and Figure 4.8, it was determined that the reduction in bypass nozzle exit velocity was due to the variation in total pressure and total temperature at the bypass nozzle exit plane. This lead to the analysis described in Section 4.4.4 in which the location of the source(s) of the total temperature and total pressure variations was sought.

\subsubsection{Distortion Attenuation}

It is also interesting to investigate the magnitude of the attenuation of the distortion through the fan and bypass duct. As discussed in Section 1.2, the negative slope of the fan characteristic tends to attenuate total pressure distortion, causing the total pressure profile to be more uniform downstream of the fan. Although the distorted total pressure profile persists, it is reduced in magnitude and rotated by the action of the fan and bypass duct. This effect can be seen in plots of ring total pressure at the AIP and bypass nozzle exit plane for the distorted test, shown in Figure 4.9. The total pressure plots shown in Figure 4.9 were normalized by the ring mean total pressure at the AIP and bypass nozzle exit plane, respectively. 

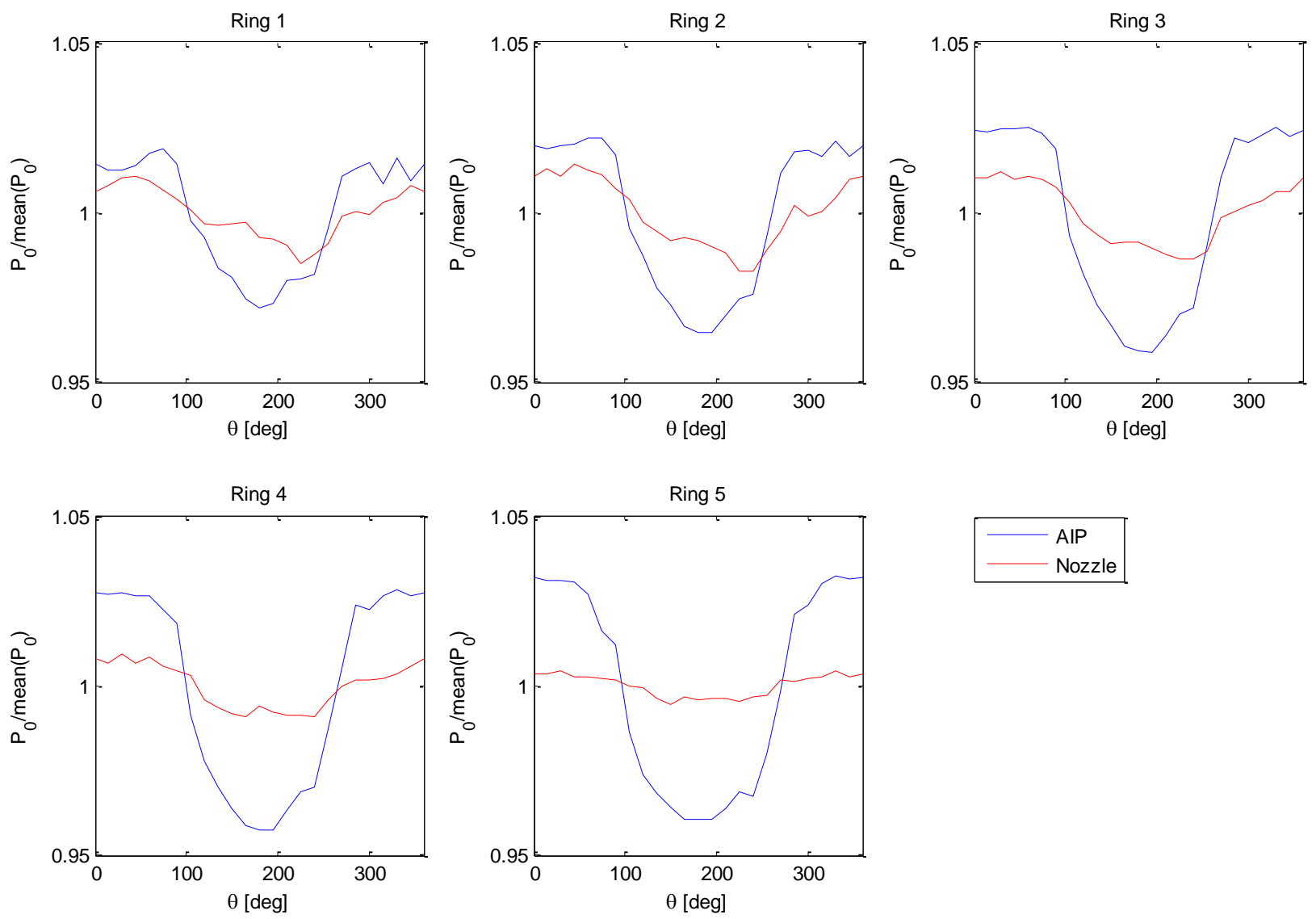

Figure 4.9: Ring Mean Normalized Total Pressure Profiles for Each Ring at AIP and Bypass

Nozzle Exit Plane for Distorted Test

As shown in Figure 4.9, as expected, the total pressure profile variation is reduced in magnitude, and is more uniform at the bypass nozzle exit plane than it is at the AIP for every ring. It is also interesting to note that the total pressure profiles become more uniform at the outer radii, which may be the result of separation near the transonic tip region of the fan. A more detailed analysis of the Ring 5 flow can be found in Section 4.4.4.

Another way to analyze the total pressure distortion attenuation through the fan and bypass duct is to plot the distortion indices discussed in Section 3.1 at both the AIP and the bypass nozzle exit plane for the distorted test. Plots of the circumferential distortion and extent at the AIP and bypass nozzle exit plane are shown in Figure 4.10. 


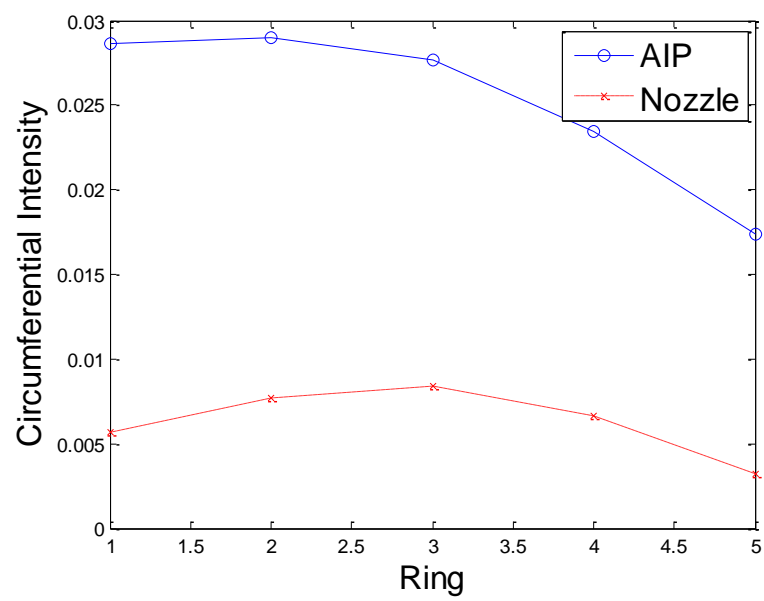

(a)

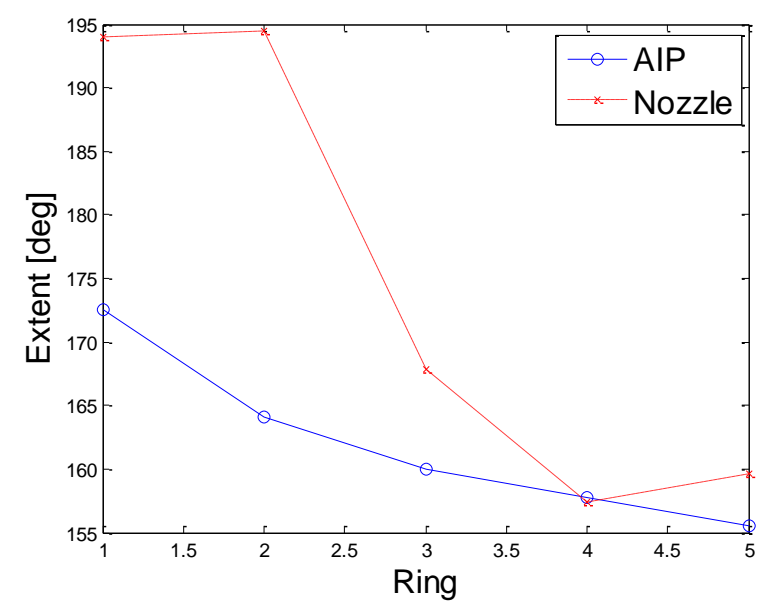

(b)

Figure 4.10: (a) Circumferential Intensity and (b) Extent at AIP and Bypass Nozzle Exit Plane for Distorted Test

As shown in Figure 4.10, the circumferential intensity decreases significantly between the AIP and bypass nozzle exit plane, while the extent increases between the AIP and bypass nozzle exit plane. The decrease in circumferential intensity in Figure 4.10 (a) further confirms expectations that the fan and bypass duct will tend to make the total pressure profile more uniform. The increase in extent shown in Figure 4.10 (b) was also expected, as the rotation of the fan will tend to smear the total pressure profile over a wider circumferential percentage of the annulus.

To further emphasize the amount of total pressure distortion attenuation between the AIP and bypass nozzle exit plane, a plot of the percent decrease in circumferential extent between the AIP and bypass nozzle exit plane was created, as shown in Figure 4.11.

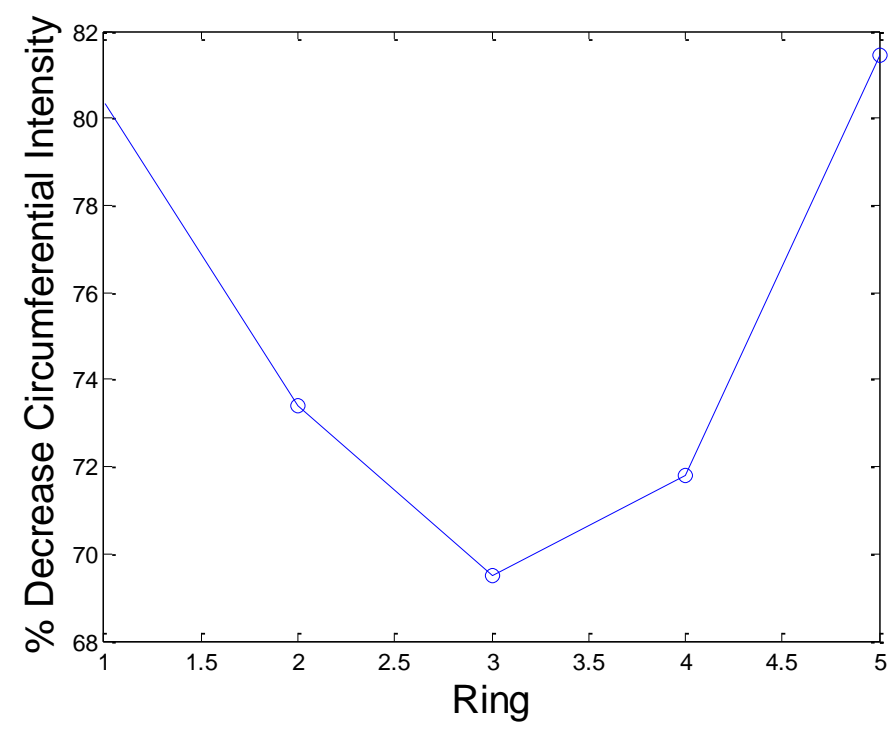

Figure 4.11: Percent Decrease in Circumferential Intensity from AIP to Bypass Nozzle Exit Plane for Distorted Test 
As shown in Figure 4.11, the circumferential intensity decreases by $69.5 \%-81.5 \%$ between the AIP and bypass nozzle exit plane. Thus the fan and bypass duct decreased the circumferential intensity by almost an order of magnitude. This substantial decrease indicates that from a total pressure point of view, the fan works to improve the flow variation.

\subsubsection{Entropy Generation and Efficiency}

Losses in total pressure and increases in total temperature are directly related to entropy generation by the Second Law of Thermodynamics. Based on measurements taken at the AIP and bypass nozzle exit, as well as ambient conditions, entropy generation throughout the bypass flow path can be calculated. The methodology of this calculation is described in Section 3.3, but the schematic shown in Figure 3.3 will be reproduced here for clarity.

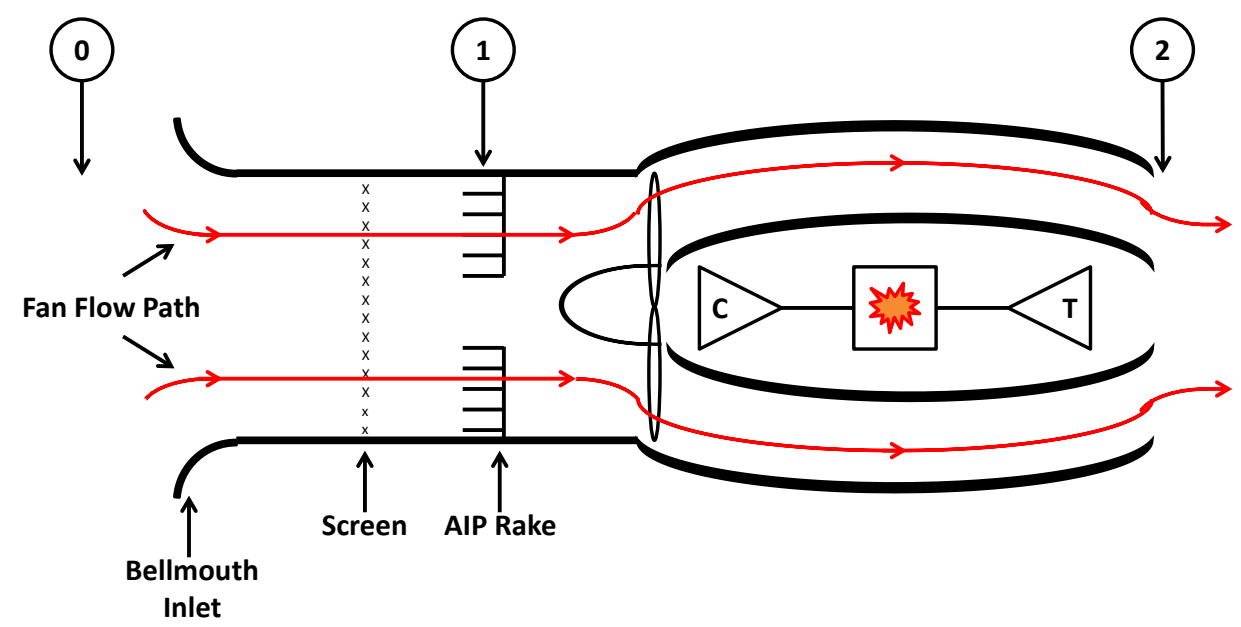

Figure 4.12: Flow Schematic for Entropy Generation and Efficiency Calculations

The entropy produced along the fan flow path from Station 0 to Station 2 was calculated for both clean and distorted tests at every measurement point using Equation 3.28, reproduced below.

$$
\Delta s_{j k, i}=c_{p} \ln \left(\frac{T_{0 i, k}}{T_{0 i, j}}\right)-R \ln \left(\frac{p_{0 i, k}}{p_{0 i, j}}\right)
$$

The entropy produced between Stations 0 and 2 at every measurement point was then massaveraged for both clean and distorted tests to provide a representative value of the entropy produced along the fan flow path for both tests. The presence of the distortion screen caused the mass-averaged entropy production over the entire fan flow path to increase from a clean test value of $41.45 \mathrm{~J} / \mathrm{kg} / \mathrm{K} \pm 7.2 \mathrm{~J} / \mathrm{kg} / \mathrm{K}$ to $51.27 \mathrm{~J} / \mathrm{kg} / \mathrm{K} \pm 7.2 \mathrm{~J} / \mathrm{kg} / \mathrm{K}$ for the distorted test, a nominal $23.68 \%$ increase. This indicates that the presence of the BLI distortion increased the losses over the entire fan flow path. A more detailed uncertainty analysis can be found in Appendix B. This increase in entropy generation, and thus system losses, helps explain the stream thrust loss and TSFC increase described in Section 4.3. 
With the entire fan flow path entropy generation calculated, it was desired that the entropy generated between Stations 0 and 1 be compared to the entropy generated between Stations 0 an 2. Since the major sources of losses between Station 1 and 2 are the fan and bypass duct, this analysis would provide an estimate of how the fan and bypass duct process the distortion. As described in Section 3.1, instrumentation limitations prevented the calculation of a mass averaged entropy generation between Stations 0 and 1. Instead, plots of the entropy generated from Station 0 to each ring of the bypass region of the AIP were created. Similarly, plots of the entropy generated from Station 0 to each ring of the bypass nozzle exit plane were created. Figure 4.13 shows the unwrap plot of the entropy produced between Station 0 and each ring at the bypass nozzle exit plane.
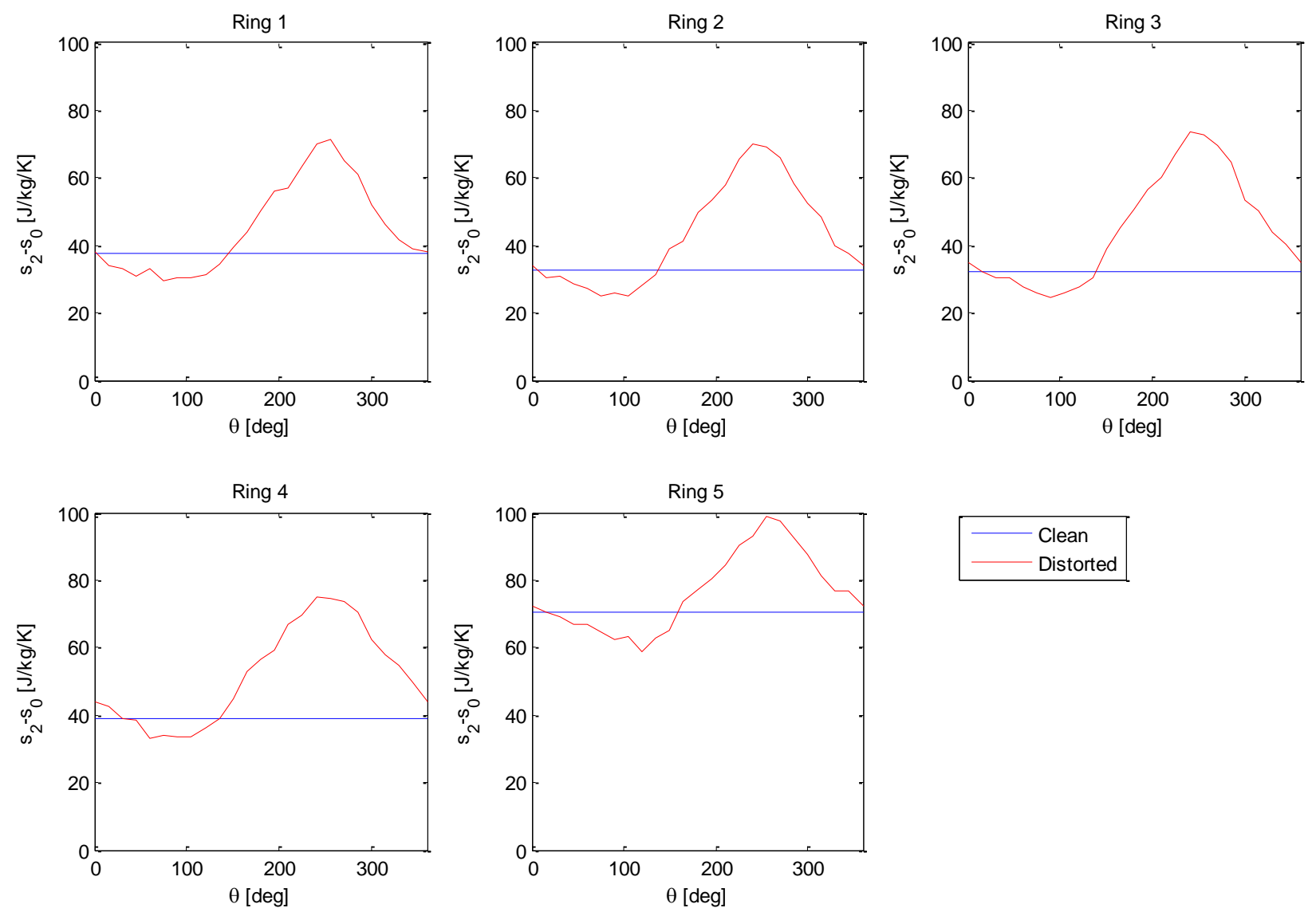

Figure 4.13: Entropy Generated From Station 0 to Station 2 for Each Ring Measurement Stations Marked by Blue Dashed Line

As shown in Figure 4.13, there is one portion of the annulus for each ring in which there is less entropy production for the distorted test than for the clean test. For the rest of the annulus, however, there is much more entropy production for the distorted test compared to the clean test. The magnitude and extent of the low entropy production region is much smaller than that of the high entropy production region, resulting in the mass-averaged $23.68 \%$ increase in entropy production for the distorted test described above. It is also interesting to note that the entropy production increases substantially between Ring 4 and Ring 5 (the two outermost rings). This may be indicative of a combination of transonic effects in the fan and growth of the boundary layer, as will be discussed below. 
In order to draw inferences on how the distortion was processed by the fan and bypass duct, a plot of the entropy generated from Station 0 to ring 2 of the AIP was created for each ring, shown in Figure 4.14.
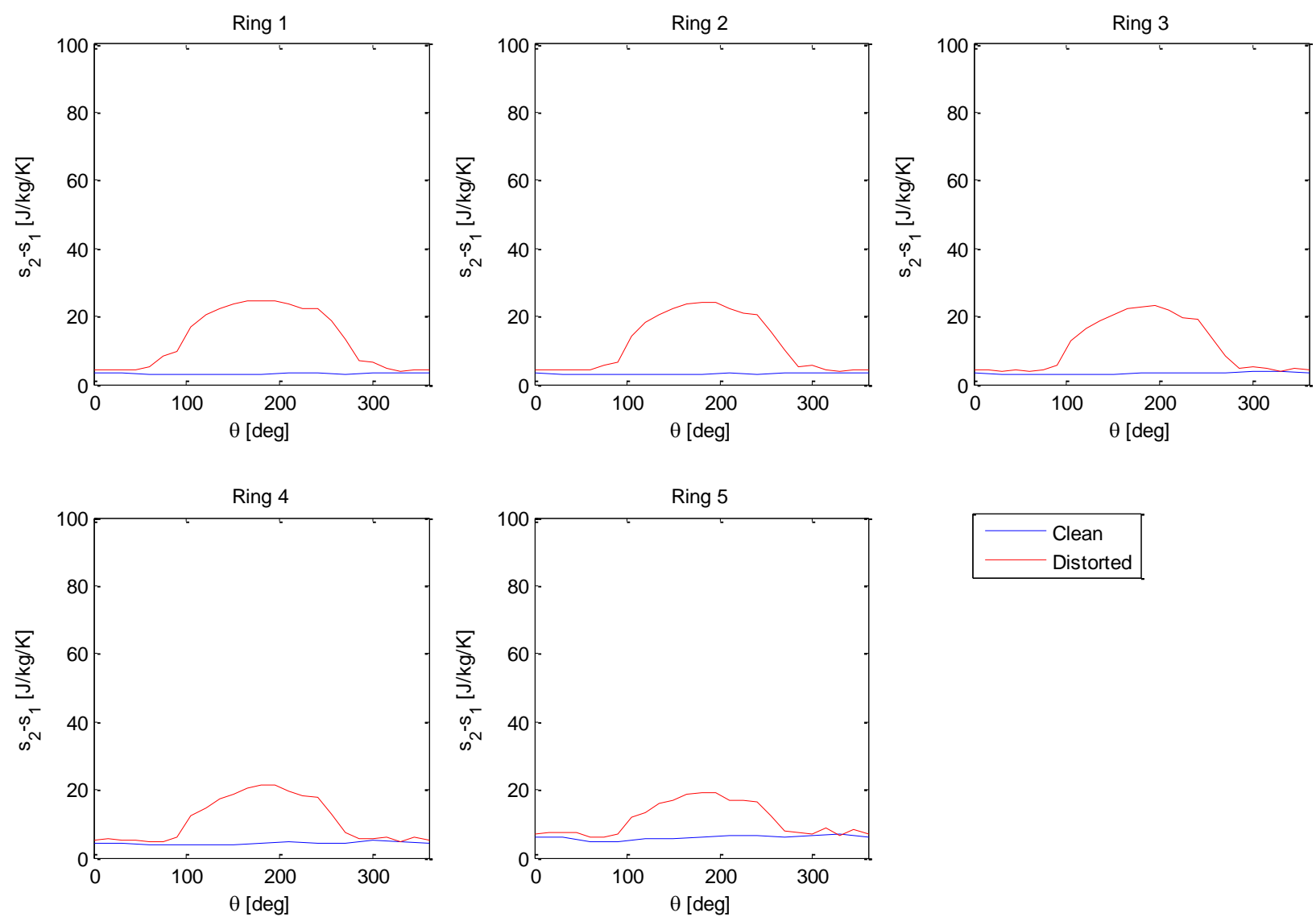

Figure 4.14: Entropy Generated from Station 0 to Station 1 for Ring 2 Measurement Stations

Marked by Blue Dashed Line

Upon comparison of Figure 4.14 with Figure 4.13, it can be seen that the majority of the entropy generated over the entire fan flow path is created between the AIP and the bypass nozzle exit plane for both clean and distorted tests. It is also important to note that the entropy produced at the AIP for every ring is of roughly the same magnitude for both clean and distorted tests. This trend led to the conclusion that the increased entropy generation seen in Ring 5 in Figure 4.13 is due to effects of the fan and boundary layer. For the fan speed at which the clean and distorted tests were performed, the fan is transonic towards the tip, which increases the loading on the blade. The presence of the distortion further increases the blade loading, resulting in a very inefficient, or "lossy" flow region. While the exact cause of the increased entropy generation for Ring 5 cannot be determined with the current set of measurements, the point remains that there is significantly more entropy production at the outer ring. Thus, in an effort to avoid increasing the losses of an already lossy region, distortion should be located away from the tip of a fan.

To further analyze the difference in entropy production between Stations 0 and 2 and Stations 0 and 1 the extreme values in Figure 4.13 were compared to the extreme values in Figure 4.14. 
Due to the similarities in entropy production trends for all rings, the extreme values of entropy production were compared for Ring 2. Similar trends exist for all rings. The difference between all possible differences between the extreme values in Figure 4.13 and Figure 4.14 are shown graphically in Figure 4.15 and are summarized in Table 4.3. The four sets of bars shown in Figure 4.15 bound all possible entropy generation values between the AIP and the bypass nozzle exit plane. Bar set 1 represents the worst entropy generation streamtube at the AIP becoming the best entropy generation streamtube at the bypass nozzle exit. This set of bars shows the smallest possible entropy generation between the AIP and the bypass nozzle exit plane. Bar set 2 represents the best entropy generation streamtube at the AIP becoming the worst entropy generation streamtube at the bypass nozzle exit. This set of bars shows the largest possible entropy generation between the AIP and the bypass nozzle exit. The entropy generation differences at bar sets 1 and 2 bound the entropy production for all possible combinations of streamtubes between the AIP and bypass nozzle exit. Bar set 3 represents the best entropy generation streamtube at the AIP becoming the best entropy generation streamtube at the bypass nozzle exit. Similarly, bar set 4 represents the worst entropy generation streamtube at the AIP becoming the worst entropy generation streamtube at the bypass nozzle exit.

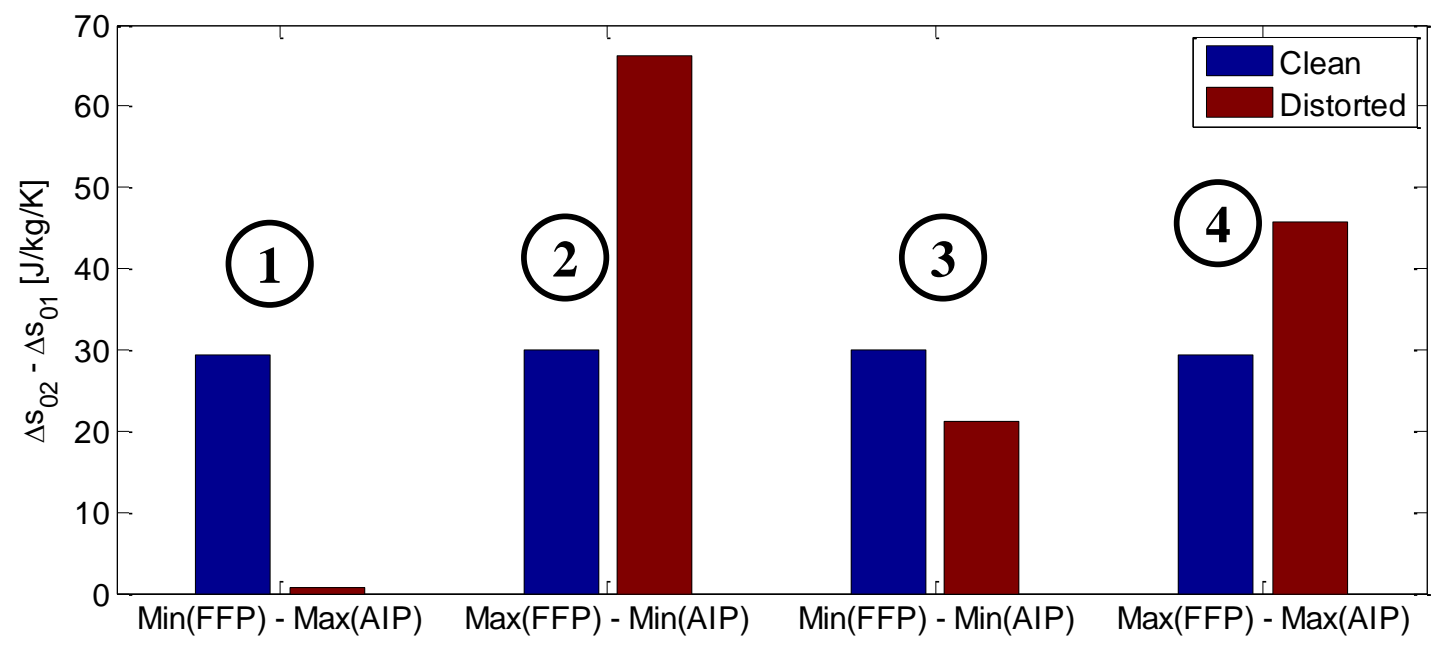

Figure 4.15: Entropy Production Change For Clean and Distorted Tests for Ring 2

Table 4.3: Summary of Figure 4.15

\begin{tabular}{|c|c|c|c|c|}
\hline \multirow{2}{*}{} & \multicolumn{4}{|c|}{ Entropy Generation [J/kg/K] } \\
\cline { 2 - 5 } & Bar Set 1 & Bar Set 2 & Bar Set 3 & Bar Set 4 \\
\hline Clean & 29.29 & 29.91 & 29.91 & 29.29 \\
\hline Distorted & 0.64 & 66.22 & 21.11 & 45.75 \\
\hline Difference & -28.65 & 36.31 & -8.79 & 16.45 \\
\hline
\end{tabular}

As shown in Figure 4.15 and Table 4.3, the entropy generation between the AIP and the bypass nozzle exit plane is relatively constant, as expected, for the clean inlet test. For the distorted test, on the other hand, the entropy generation between the AIP and bypass nozzle exit plane varies greatly. Comparing bar sets 1 and 2 shows that for the distorted test, the entropy generation between the AIP and the bypass nozzle exit plane could vary from $0.64 \mathrm{~J} / \mathrm{kg} / \mathrm{K}$ to $66.22 \mathrm{~J} / \mathrm{kg} / \mathrm{K}$. This large variation indicates the wide range of performance of the fan and bypass duct in the 
presence of embedded BLI-type distortion. This trend is further confirmed in comparing bar sets 3 and 4. Based on these comparisons, a region of flow must exist between the AIP and the bypass nozzle exit in which there is less entropy generation for the distorted test than for the clean test. Similarly, a region of flow must exist between the AIP and the bypass nozzle exit in which there is more entropy production for the distorted test than for the clean test. These decreases and increases in entropy production indicate the presence of regions of higher efficiency and lower efficiency, respectively. Thus, while the entropy production between the AIP and the bypass nozzle exit plane cannot be exactly calculated, there must exist some mechanism that reduces the entropy production in some region, while increasing the entropy production in another region. Therefore, different regions of the fan and bypass duct are affected by the presence of the distortion in different ways.

The fan flow path efficiency was also computed to analyze the effect of the distortion on the entire fan flow path. Using Equation 3.30, reproduced below, the fan flow path efficiency was calculated for every measurement point at the bypass nozzle exit plane.

$$
\eta_{f f p}=\frac{\left(\left(\frac{p_{02}}{p_{a t m}}\right)^{\frac{\gamma-1}{\gamma}}-1\right)}{\left(\frac{T_{02}}{T_{a t m}}-1\right)}
$$

These values were then mass-averaged, yielding a single value for the fan flow path efficiency for both clean and distorted tests. For the clean test, the fan flow path efficiency was calculated to be $60.15 \% \pm 3.94 \%$. For the distorted test, the fan flow path efficiency was calculated to be $50.58 \% \pm 4.42 \%$. Thus the presence of distortion resulted in a nominal $9.57 \%$ reduction in fan flow path efficiency. It should be noted that while the uncertainty in this calculation is fairly high, the overall trend of a reduction in fan flow path efficiency due to distortion holds true. A more detailed uncertainty analysis can be found in Appendix B, demonstrating the need for highly accurate temperature measurements.

The fan flow path efficiency is not to be mistaken for the fan efficiency, as the measurements used in the calculation of the fan flow path efficiency include the effect of the inlet, distortion screen, AIP rake, fan, and bypass duct. The reduction in fan flow path efficiency agrees with the studies described in Section 1.2, although this reduction in efficiency is much larger than the efficiency reductions predicted in those studies. According to a study done by Florea, et al, the distortion produced by NASA's Inlet A is predicted to result in a decrease in fan efficiency of up to $6 \%$. The $9.57 \%$ reduction in fan flow path efficiency described above would agree with that prediction, with the additional losses likely due to the presence of the AIP rake and mixing losses in the engine ducts.

To further analyze the effect of distortion, the fan flow path efficiency for each measurement point was also plotted for each ring, similar to the entropy generation plot shown in Figure 4.13. The fan efficiency profile for each ring is shown in Figure 4.16. 

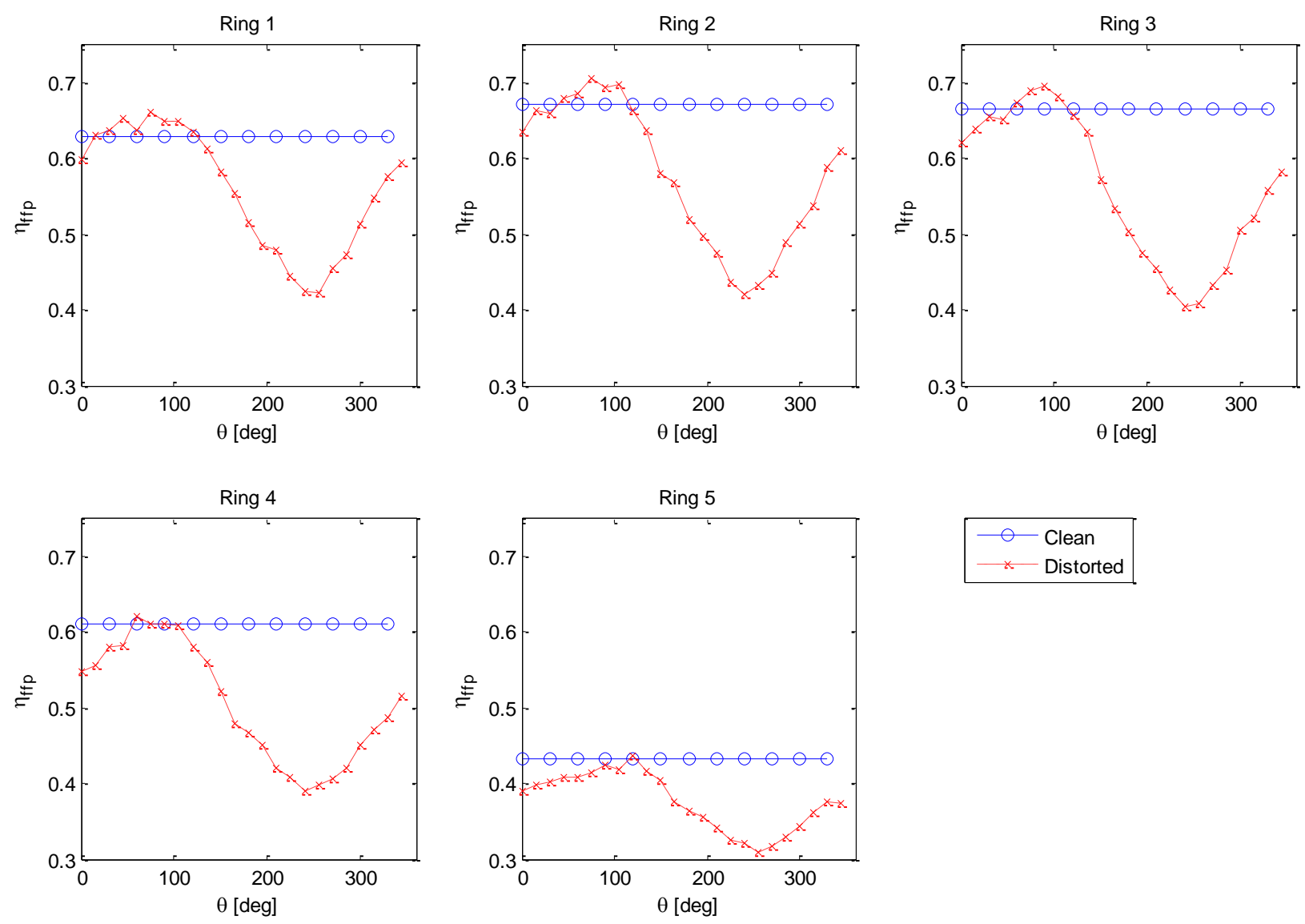

Figure 4.16: Fan Flow Path Efficiency for Each Ring at the Bypass Nozzle Exit Plane

As shown in Figure 4.16, there were regions of higher and lower fan flow path efficiency for the distorted test, while the clean test fan flow path efficiency remained constant across each ring. The same trends that were discussed with the entropy production curves in Figure 4.13 are evident in Figure 4.16, but in the opposite sense. For example, the entropy production increased greatly between Rings 4 and 5 in Figure 4.13, while the fan flow path efficiency decreased greatly between Rings 4 and 5 in Figure 4.16. These trends can be further explained by plotting the fan flow path efficiency and entropy generation for a given ring together, along with raw total temperature and total pressure measurements all for the distorted test, as shown in Figure 4.17. The curves in Figure 4.17 are for Ring 2 only, as all rings exhibited similar trends. 

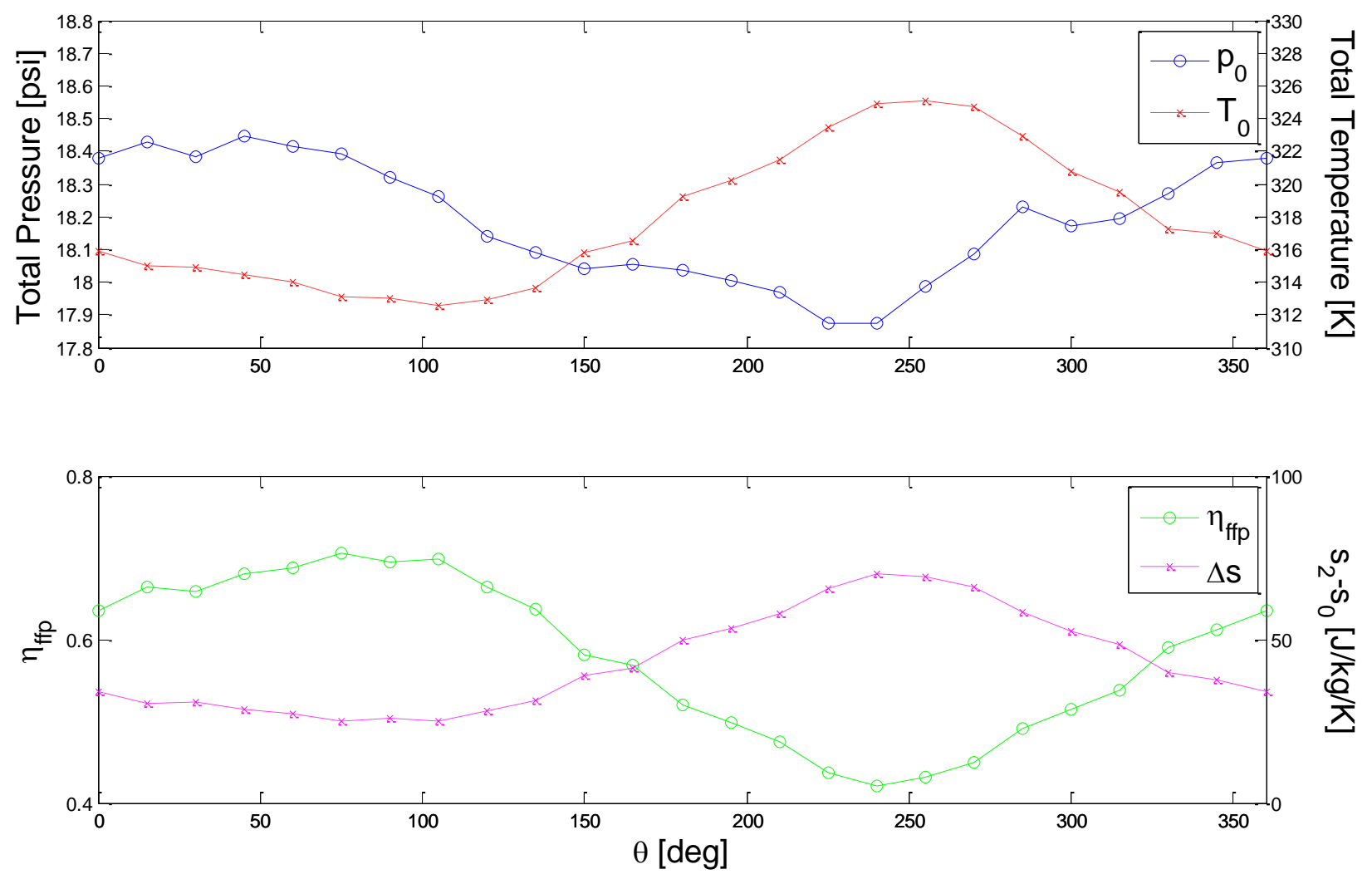

Figure 4.17: Comparison of Raw Measurements with Calculated Fan Flow Path Efficiency and Entropy Generation for Ring 2 for Distorted Test

As shown in Figure 4.17, regions of high total pressure and low total temperature correspond to regions of high fan flow path efficiency and low entropy production, and vice versa. This trend is expected, as regions of high total pressure at the AIP result in regions of high total pressure downstream of the fan and vice versa. While the fan will work to attenuate the total pressure distortion, a high total pressure region at the AIP will still result in a higher total pressure region downstream of the fan. Thus, the regions of high pressure shown in Figure 4.17 likely correspond to regions of high total pressure at the AIP. Therefore, as Figure 4.17 shows, the presence of distortion (i.e., low total pressure) results in regions of low efficiency and high entropy production. It should be noted that the magnitude of the parameters shown in Figure 4.17 cannot be attributed entirely to the fan. These values are the combined effect of losses due to the distortion screen and the fan's response to these losses.

Another interesting result comes from a comparison of the max-to-min variation in the curves shown in Figure 4.17. To perform this comparison, the maximum and minimum values of each curve were normalized by their mass-averaged values. The difference between these normalized values provides the percent variation of each parameter relative to its mass-averaged value, which is shown in Figure 4.18. It should be noted that the variation of total pressure and fan flow path efficiency 


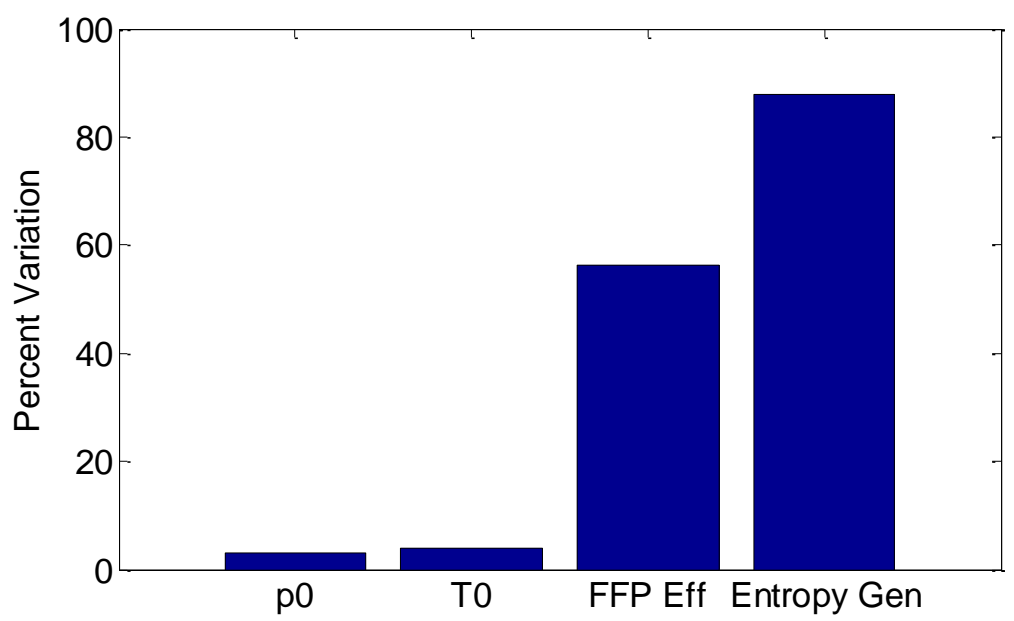

Figure 4.18: Percent Variation in Pressure, Temperature, Entropy Generation, and Fan Flow Path Efficiency at Bypass Nozzle Exit for Ring 2 Distorted Test

As shown in Figure 4.18, small variations in total pressure and total temperature result in very large variations in fan flow path efficiency and entropy generation. This trend indicates that small improvements in total pressure and total temperature variation will correspond to large improvements in fan flow path efficiency and entropy generation. It should be noted that the opposite trend also holds, i.e., small losses in total pressure and total temperature will result in large losses of fan flow path efficiency and entropy generation.

\subsubsection{Discussion of Results}

A summary of the results described above can be found in Table 4.4.

Table 4.4: Summary of Results

\begin{tabular}{|c|c|c|}
\hline Parameter & Clean & Distorted \\
\hline AIP Average $\mathrm{R}_{\mathrm{p}}$ & 0.987 & 0.954 \\
\hline Corrected Stream Thrust & $1058.44 \mathrm{lbf}$ & $894.26 \mathrm{lbf}$ \\
\hline Corrected TSFC & $0.7005 \mathrm{lbm} / \mathrm{hr} / \mathrm{lbf}$ & $0.7975 \mathrm{lbm} / \mathrm{hr} / \mathrm{lbf}$ \\
\hline Fuel Consumption & $109.82 \mathrm{GPH}$ & $104.83 \mathrm{GPH}$ \\
\hline${\text { Bypass Nozzle Average } \mathrm{R}_{\mathrm{p}}}^{\text {Bypass Nozzle Average } \mathrm{p}_{\mathrm{norm}}}$ & 1.255 & 1.208 \\
\hline Bypass Nozzle Average $\mathrm{T}_{0, \mathrm{norm}}$ & 0.994 & 1 \\
\hline Distortion Attenuation & 1.115 & 1.114 \\
\hline Mass-Averaged Entropy Generation Stations $0-2$ & $41.45 \mathrm{~J} / \mathrm{kg} / \mathrm{K}$ & $51.27 \mathrm{~J} / \mathrm{kg} / \mathrm{K}$ \\
\hline Minimum Entropy Production Difference & $\mathrm{N} / \mathrm{A}$ & $-8.79 \mathrm{~J} / \mathrm{kg} / \mathrm{K}$ \\
\hline Maximum Entropy Production Difference & $\mathrm{N} / \mathrm{A}$ & $16.45 \mathrm{~J} / \mathrm{kg} / \mathrm{K}$ \\
\hline Mass-Averaged Fan Flow Path Efficiency & $60.15 \%$ & $50.58 \%$ \\
\hline
\end{tabular}

As shown in Table 4.4, the presence of the distortion screen resulted in a decrease in average total pressure at the AIP. The distortion produced by the distortion screen also affected the 
modified JT15D-1 engine's performance, as the corrected stream thrust decreased and corrected TSFC increased in the presence of distortion. Despite these performance losses, the bulk fuel consumption of the engine decreased in the presence of distortion, indicating that if the loss in stream thrust is not too drastic, a potential for fuel consumption savings exists for embedded BLI applications if the required thrust reduction exceeds the reduced thrust produced by the engine.

The presence of the distortion screen also created a decrease in the average total pressure ratio at the bypass nozzle exit plane. This decrease in total pressure ratio is due to the decreased AIP average total pressure recovery in the presence of distortion. It should be noted that not only did the average total pressure ratio at the bypass nozzle exit plane decrease, the total pressure ratio profile varied around the nozzle annulus in the presence of distortion. At the bypass nozzle exit plane, the average normalized static pressure and average normalized total temperature remained constant between the clean and distorted tests. It should be noted that, while the average normalized total temperature did not vary significantly between the clean and distorted tests, the local variation in normalized total temperature did vary in the presence of distortion.

An analysis of the total pressure profiles between the AIP and the bypass nozzle showed that there was significant attenuation of the distortion by the fan and bypass duct. The circumferential intensity at the bypass nozzle was $69.5 \%-81.5 \%$ lower than that at the AIP.

An investigation into the losses along the fan flow path showed that more entropy was produced along the entire fan flow path during the distorted test than during the clean test. This increase in mass-averaged entropy production was directly related to a decrease in the mass-averaged fan flow path efficiency. A study of the entropy production change relative to atmospheric conditions for each ring at the bypass nozzle exit and AIP showed that the majority of entropy production occurs in the fan and bypass duct. The entropy production profile for each ring showed that between the AIP and the bypass nozzle exit duct, a small region of lower entropy production compared to the clean test existed, along with a much larger region of higher entropy production compared to the clean test. Thus, the fan and bypass duct must process the different distortion regions in different ways. Another important observation from the entropy production along the entire fan flow path for each ring is that the entropy production increases significantly at the outermost radius. This is indicative of increased losses due to transonic effects at the fan combined with losses in the boundary layer between the fan and bypass nozzle exit. Thus, it was concluded that distortion should be located away from the tip in order to minimize losses in a very lossy area.

Finally, by comparing the bypass nozzle exit plane total pressure and total temperature profiles around a ring with the entropy production and fan flow path efficiency profiles around the same ring, it was found that regions of high total pressure correspond to regions of low total temperature, which result in high fan flow path efficiency and low entropy production. The opposite trend is also true. An analysis of the magnitude of the max-to-min variation in each of these profiles showed that small perturbations in total pressure and total temperature result in very large perturbations in fan flow path efficiency and entropy production. Therefore, very small improvements in total pressure variation and total temperature variation will result in very large improvements in fan flow path efficiency and entropy generation. 


\section{Conclusions and Recommendations}

\subsection{Conclusions}

An extensive review of literature pertaining to engine performance in the presence of inlet flow distortion yielded no experimental studies into the effect of distortions typical to boundary layer ingesting (BLI) engines on engine performance. Therefore, an investigation into the performance losses resulting from inlet flow distortion typical in BLI engines was performed. A modified JT15D-1 turbofan engine was used in this study, with a distortion screen designed to replicate the total pressure profile of NASA Inlet A installed in the engine's inlet duct. Results of this investigation showed a $15.5 \%$ decrease in corrected stream thrust and a $14 \%$ increase in corrected TSFC in the presence of embedded BLI-type distortion.

Flow measurements made at the bypass nozzle exit plane allowed for the calculation of the entropy production along the entire fan flow path and fan flow path efficiency for both clean and distorted tests. The mass-averaged entropy production along the fan flow path increased by $24 \%$ for the distorted test, while the fan flow path efficiency decreased by $9.5 \%$ for the same test. Unwrap plots of the entropy production and fan flow path efficiency led to the following conclusions.

- For both clean and distorted tests, the most significant increase in entropy production occurred between the AIP and the bypass nozzle exit plane. This indicates that the fan and bypass duct are major contributors to flow losses, made even worse in some regions due to the embedded BLI-type distortion.

- The entropy generation increases significantly towards the outermost radii at the bypass nozzle exit. The fan flow path efficiency at these radii also decreases significantly. Therefore, in order to minimize losses in a very lossy region, inlet flow distortion should be located away from the tip region of the fan.

- At the bypass nozzle exit plane, regions of high total pressure and low total temperature correspond to regions of high fan flow path efficiency and low entropy generation. It was also found that small perturbations in total pressure variation and total temperature variation result in very large perturbations in fan flow path efficiency and entropy generation. Thus, small improvements in total pressure variation and total temperature variation will result in very large improvements in fan flow path efficiency and entropy generation. The opposite trend will also hold true.

- The difference in max-to-min entropy generation for a given ring increases significantly in the presence of inlet distortion. Compared to the clean test, there exists a small region of lower entropy production for the distorted test, and a much larger region of higher entropy production for the distorted test. Thus, depending on the location within the distorted region, the fan and bypass duct will respond in different ways. This demonstrates the need for integrated airframe/inlet/engine design to minimize losses in the entire system. 


\subsection{Recommendations}

All of the following recommendations would assist in locating trouble areas within a turbofan engine that would need to be investigated in detail and/or redesigned for use in BLI applications.

1. A study of the performance of the modified JT15D-1 turbofan engine subjected to various BLI-type inlet distortions at multiple engine speeds would provide further evidence to support or dispute the conclusions reached in this thesis. Tests of the performance of the modified JT15D-1 engine at multiple speeds for a given distortion profile would allow for the assessment of the engine's performance through multiple operating points. Knowledge of the engine's nominal operating map could lead to an analysis as to the extent a given distortion will change the engine's operating point relative to its nominal operating point.

2. Engine tests using various distortion screens with differing distortion indices would potentially allow for a correlation between distortion indices and engine performance. This correlation would be similar to ARP 1420, except from a whole engine performance perspective.

3. In order to more fully characterize the entropy generated throughout the fan flow path, knowledge of the static pressure or static temperature field at the AIP would allow for the mass flow distribution at the AIP to be computed absolutely. This would allow for the entropy generation at the AIP to be mass averaged and compared not only for both clean and distorted cases, but also to the entropy generated for the entire fan flow path.

4. Detailed flow measurements made directly behind the fan would help in separating the effects of the fan from the effects of the bypass duct. Combined with recommendation $\# 1$, these fan flow measurements would help in determining the entropy produced between the AIP and the fan exit plane. From there, the flow measurements made at the bypass nozzle exit plane could be used to determine the mixing losses through the bypass duct. Flow measurements made behind the fan outlet guide vanes would further describe the effect and propagation of distortion through the entire fan flow path.

5. Increased accuracy of temperature measurements at the bypass nozzle exit plane would lead to decreases in the uncertainty in fan flow path efficiency and entropy generation. The calculation of these parameters is very important in an analysis of losses through the entire engine system, so decreased uncertainty would help in the location of trouble areas in an engine. 


\section{References}

${ }^{1}$ Del Rosario, R., Koudelka, J., Wahls, R., and Madavan, N. "NASA Subsonic Fixed Wing Project Overview." NASA Fundamental Aeronautics Program 2012 Technical Conference, March 2012.

${ }^{2}$ Ferrar, A. M. and O’Brien, W. F. "Progress in Boundary Layer Ingesting Embedded Engine Research." 48th AIAA/ASME/SAE/ASEE Joint Propulsion Conference \& Exhibit. No. AIAA 2012-4283, AIAA, August 2012.

${ }^{3}$ Ferrar, A. Measurements of Flow in Boundary Layer Ingesting Serpentine Inlets. Master's Thesis. Virginia Polytechnic Institute and State University, Blacksburg, VA, December 2011.

${ }^{4}$ Anabtawi, A. J., Blackwelder, R., Liebeck, R., and Lissaman, P. "Experimental Investigation of Boundary Layer Ingesting Diffusers of a Semi-Circular Cross Section." 36th Aerospace Sciences Meeting \& Exhibit. No. AIAA-98-16743, AIAA, January 1998.

${ }^{5}$ Conrad, E. W., and Sobolewski, A. E. "Investigation of Effects of Inlet-Air Velocity Distortion on Performance of Turbojet Engine." NACA RM ESOGIl, September 1950.

${ }^{6}$ Wallner, L. E., Conrad, E. W., and Prince, W. R. "Effect of Uneven Airflow Distribution to the Twin Inlets of an Axial-Flow Turbojet Engine." NACA RM E52K06, January 1953.

${ }^{7}$ Conrad, E. W., Hanson, M. P., and McAulay, J. E. "Effects of Inlet-Air-Flow Distortion on Steady-State Altitude Performance of an Axial-Flow Turbojet Engine." NACA RM E55A04, September 1955.

${ }^{8}$ Russey, R. E. and Seashore, F. L. "Effects of Inlet Air Distortion on Steady-State Performance of an Axial Flow Turbojet Engine." NACA RM E57L31, January 1958.

9 Plourde, G. A. and Stenning, A. H. "Attenuation of Circumferential Inlet Distortion in Multistage Axial Compressors." Journal of Aircraft. Vol. 5, No. 3, February 1968, pp. 236-242.

${ }^{10}$ Walter, W. A. and Shaw, M. "Predicted F100 Engine Response to Circumferential Pressure and Temperature Distortion." $15^{\text {th }}$ AIAA/SAE/ASME Joint Propulsion Conference. No. AIAA-79-1310, AIAA, June 1979.

${ }^{11}$ Soeder, R. H. and Bobula, G. A. "Effect of Steady-State Pressure Distortion on Inlet Flow to a High-Bypass-Ratio Turbofan Engine.” NASA Technical Memorandum 82964, October 1982. 
${ }^{12}$ Katsiopoulos, P., Anastasiou, S., Lekas, T. I., Kottarakos, A., Haslam, A. S., and Pilidis, P. "Influence of Degradation and Inlet Distortion on Gas Turbine Performance." International Journal of Turbo and Jet Engines. Vol. 21, 2004, pp. 47-55.

${ }^{13}$ Daggett, D. L., Kawai, R., and Friedman, D. "Blended Wing Body Systems Studies: Boundary Layer Ingestion Inlets With Active Flow Control." NASA Contractor Report. No. NASA/CR-2003-212670, December 2003.

14 Tillman, T. G. "System Study and Distortion-Tolerant Fan Design for a Boundary Layer Ingesting Propulsion System," NASA Contractor Report. No. NASA NRA NNC07CB59C Phase 2 Final Report, July 2010.

${ }^{15}$ Hardin, L. W., Tillman, G., Sharma, O. P., Berton, J., and Arend, D. J. "Aircraft System Study of Boundary Layer Ingesting Propulsion." 48th AIAA/ASME/SAE/ASEE Joint Propulsion Conference. AIAA, August 2012.

${ }^{16}$ Kawai, R. T., Friedman, D. M., and Serrano, L. "Blended Wing Body (BWB) Boundary Layer Ingestion (BLI) Inlet Configuration and System Studies." NASA Contractor Report. No. NASA/CR-2006-214534, December 2006.

${ }^{17}$ Gladin, J. C., Sands, J. S., Kestner, B. K., and Mavris, D. N. "Effects of Boundary Layer Ingesting (BLI) Propulsion Systems on Engine Cycle Selection and HWB Vehicle Sizing." 50th AIAA Aerospace Sciences Meeting. AIAA-2012-0837, AIAA, January 2012.

${ }^{18}$ Plas, A. P., Sargeant, M. A., Madani, V., Crichton, D., Greitzer, E. M., Hynes, T. P., and Hall, C. A. "Performance of a Boundary Layer Ingesting (BLI) Propulsion System." $45^{\text {th }}$ AIAA Aerospace Sciences Meeting. No. AIAA-2007-450, AIAA, January 2007.

${ }^{19}$ SAE International, S-16 Committee, “Gas Turbine Inlet Flow Distortion Guidelines,” January 2001, Aerospace Recommended Practice 1420.

${ }^{20}$ Ferrar, A. M. and O'Brien, W. F. "Fan Response to Total Pressure Distortions Produced by Boundary Layer Ingesting Serpentine Inlets." 48th AIAA/ASME/SAE/ASEE Joint Propulsion Conference. AIAA, August 2012.

${ }^{21}$ Liebeck, R. H. “ Design of the Blended Wing Body Subsonic Transport.” Journal of Aircraft. Vol. 41 No. 1, January-February 2004, pp. 10-25.

${ }^{22}$ Florea, R. V., Matalanis, C., Hardin, L. W., Stucky, M., and Shabbir, A. "Parametric Analysis and Design for Embedded Engine Inlets." 48th AIAA/ASME/SAE/ASEE Joint Propulsion Conference. AIAA, August 2012.

23 Alhamaly, A. "Performance Analysis of a Transonic Fan." $11^{\text {th }}$ Annual Virginia Tech Undergraduate Research Conference. Blacksburg, Virginia, April 2013. 
${ }^{24}$ Yao, J., Gorrell, S. E., and Wadia, A. R. "High-Fidelity Numerical Analysis of Per-Rev-Type Inlet Distortion Transfer in Multistage Fans - Part I: Simulations with Selected Blade Rows." Journal of Turbomachinery. Vol. 132. October 2010.

${ }^{25}$ Yao, J., Gorrell, S. E., and Wadia, A. R. "High-Fidelity Numerical Analysis of Per-Rev-Type Inlet Distortion Transfer in Multistage Fans - Part II: Entire Component Simulation and Investigation". Journal of Turbomachinery. Vol. 132. October 2010. 


\section{A. Derivation of Core Mass Flow Equations}

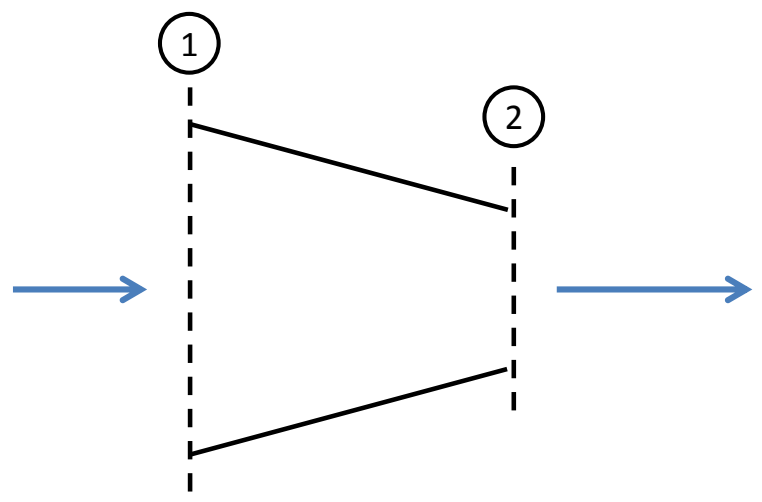

Figure A.1: Nozzle Flow Schematic

$p_{01}$ Equation:

$$
\begin{gathered}
\dot{m}_{1}=\dot{m}_{2} \\
\rho_{1} u_{1} A_{1}=\rho_{2} u_{2} A_{2}
\end{gathered}
$$

Substitute compressible flow (isentropic) relationships

$$
\frac{p_{1}}{R T_{1}} M_{1} A_{1} \sqrt{\gamma R T_{1}}=\frac{p_{2}}{R T_{2}} M_{2} A_{2} \sqrt{\gamma R T_{2}}
$$

Cancel $R$ and $\sqrt{\gamma R}$

$$
\frac{p_{1} M_{1} A_{1}}{\sqrt{T_{1}}}=\frac{p_{2} M_{2} A_{2}}{\sqrt{T_{2}}}
$$

Isentropic relationships

$$
\begin{gathered}
T=T_{0}\left(\frac{p}{p_{0}}\right)^{\frac{\gamma-1}{\gamma}} \\
M=\sqrt{\frac{2}{\gamma-1}\left(\left(\frac{p_{0}}{p}\right)^{\frac{\gamma-1}{\gamma}}-1\right)}
\end{gathered}
$$


Subsitute for $M_{1}, M_{2}, T_{1}$, and $T_{2}$

$$
\frac{p_{1} A_{1} \sqrt{\frac{2}{\gamma-1}\left(\left(\frac{p_{01}}{p_{1}}\right)^{\frac{\gamma-1}{\gamma}}-1\right)}}{\sqrt{T_{01}\left(\frac{p_{1}}{p_{01}}\right)^{\frac{\gamma-1}{\gamma}}}}=\frac{p_{2} A_{2} \sqrt{\frac{2}{\gamma-1}\left(\left(\frac{p_{02}}{p_{2}}\right)^{\frac{\gamma-1}{\gamma}}-1\right)}}{\sqrt{T_{02}\left(\frac{p_{2}}{p_{02}}\right)^{\frac{\gamma-1}{\gamma}}}}
$$

Assume constant $T_{0}$ and cancel like terms

$$
\frac{p_{1} A_{1} \sqrt{\left(\left(\frac{p_{01}}{p_{1}}\right)^{\frac{\gamma-1}{\gamma}}-1\right)}}{\sqrt{\left(\frac{p_{1}}{p_{01}}\right)^{\frac{\gamma-1}{\gamma}}}}=\frac{p_{2} A_{2} \sqrt{\left(\left(\frac{p_{02}}{p_{2}}\right)^{\frac{\gamma-1}{\gamma}}-1\right)}}{\sqrt{\left(\frac{p_{2}}{p_{02}}\right)^{\frac{\gamma-1}{\gamma}}}}
$$

Square both sides to remove radicals and simplify

$$
p_{1}^{2} A_{1}^{2}\left(\left(\frac{p_{01}}{p_{1}}\right)^{\frac{2(\gamma-1)}{\gamma}}-\left(\frac{p_{01}}{p_{1}}\right)^{\frac{\gamma-1}{\gamma}}\right)=p_{2}^{2} A_{2}^{2}\left(\left(\frac{p_{02}}{p_{2}}\right)^{\frac{2(\gamma-1)}{\gamma}}-\left(\frac{p_{02}}{p_{2}}\right)^{\frac{\gamma-1}{\gamma}}\right)
$$

Assume constant total pressure drop $\lambda$ across the nozzle

$$
\begin{aligned}
p_{02} & =\lambda p_{01} \\
p_{1}^{2} A_{1}^{2}\left(\left(\frac{p_{01}}{p_{1}}\right)^{\frac{2(\gamma-1)}{\gamma}}-\left(\frac{p_{01}}{p_{1}}\right)^{\frac{\gamma-1}{\gamma}}\right) & =p_{2}^{2} A_{2}^{2}\left(\left(\frac{\lambda p_{01}}{p_{2}}\right)^{\frac{2(\gamma-1)}{\gamma}}-\left(\frac{\lambda p_{01}}{p_{2}}\right)^{\frac{\gamma-1}{\gamma}}\right)
\end{aligned}
$$

Mass Flow Equation:

$$
\dot{m}=\rho u A
$$

Ideal gas and isentropic relationships

$$
\begin{gathered}
\rho=\frac{p}{R T} \\
u=M \sqrt{\gamma R T}
\end{gathered}
$$

Rewrite mass flow equation 


$$
\dot{m}=\frac{p}{R T} M \sqrt{\gamma R T} A
$$

\section{Rearrange}

Isentropic relationships

$$
\dot{m}=\frac{p M A \sqrt{\gamma}}{\sqrt{R T}}
$$

$$
\begin{gathered}
T=T_{0}\left(\frac{p}{p_{0}}\right)^{\frac{\gamma-1}{\gamma}} \\
M=\sqrt{\frac{2}{\gamma-1}\left(\left(\frac{p_{0}}{p}\right)^{\frac{\gamma-1}{\gamma}}-1\right)}
\end{gathered}
$$

Substitute for $T$ and $M$

$$
\dot{m}=\frac{p A \sqrt{\frac{2 \gamma}{\gamma-1}\left(\left(\frac{p_{0}}{p}\right)^{\frac{\gamma-1}{\gamma}}-1\right)}}{\sqrt{R T_{0}\left(\frac{p}{p_{0}}\right)^{\frac{\gamma-1}{\gamma}}}}
$$




\section{B. Uncertainty Analysis}

The Measurement Specialties NetScanner Model 98RK-1 pressure scanner has an accuracy of $0.05 \%$ of the total module range, with a resolution of $0.003 \%$ of the total module range. Thus, the 5 psi module has an accuracy of 0.0025 psi and a resolution of 0.00015 psi. The uncertainty of the pressure measured by the pressure scanner can be calculated using the RMS method, shown in Equation B.1 for $i$ sources of uncertainty. The uncertainty due to resolution, $u_{0}$, is defined in Equation B.2.

$$
\begin{aligned}
& u_{d}= \pm\left(\sum_{i=1}^{n} u_{i}^{2}\right)^{\frac{1}{2}} \\
& u_{0}= \pm \frac{1}{2} \text { resolution }
\end{aligned}
$$

Thus, the uncertainty of the pressure scanner measurements can be calculated as shown in Equation B.3.

$$
u_{d}= \pm \sqrt{(0.0025 \mathrm{psi})^{2}+\left(\frac{1}{2} * 0.00015 \mathrm{psi}\right)^{2}}=0.0025 \mathrm{psi}
$$

The AIP pressure measurements were collected using Omega PX139 pressure transducers connected to a National Instruments PXI-6255 multifunction DAQ card. According to the analysis described by Ferrar for the same pressure measurement system, the uncertainty of the AIP pressure measurement system is $\pm 0.046 \mathrm{psi}^{3}$. The documentation accompanying the NI 9211 when used in an NI cDAQ-9172 chassis claims a measured temperature uncertainty of \pm 2 K. The Monarch Instruments ACT-3X tachometer claims an accuracy of $0.001 \%$ of the reading, with a resolution of 1 RPM. Thus, using the RMS method described above, the maximum uncertainty of the tachometer was calculated to be \pm 0.516 RPM. Finally, the Electronics International FT180 fuel flow transducer has a linearity of $1 \%$, with a repeatability of $0.25 \%$. Thus, using the RMS method described above, the maximum uncertainty of the fuel flow meter was calculated to be $\pm 1.13 \mathrm{GPH}$.

Figure B.1 shows a sample of the error bars on the total pressure measured at the outermost AIP ring and the outermost bypass nozzle exit plane ring for the distorted test. 


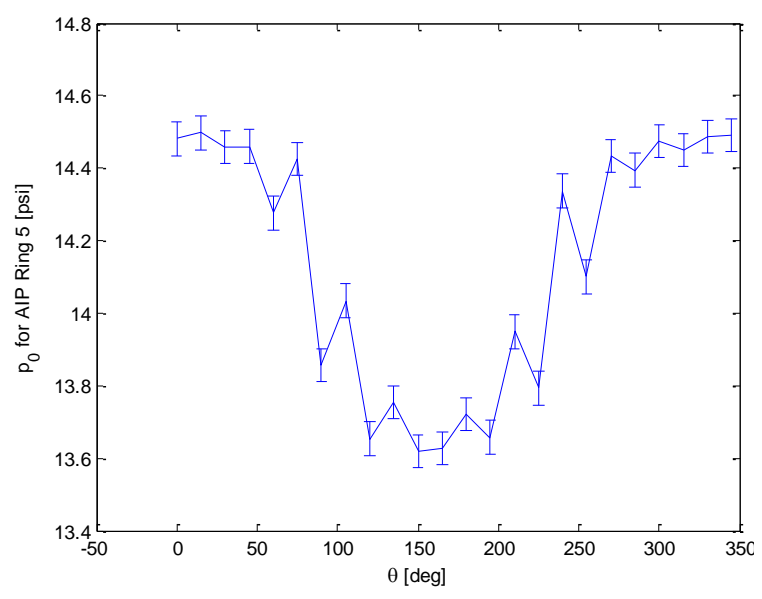

(a)

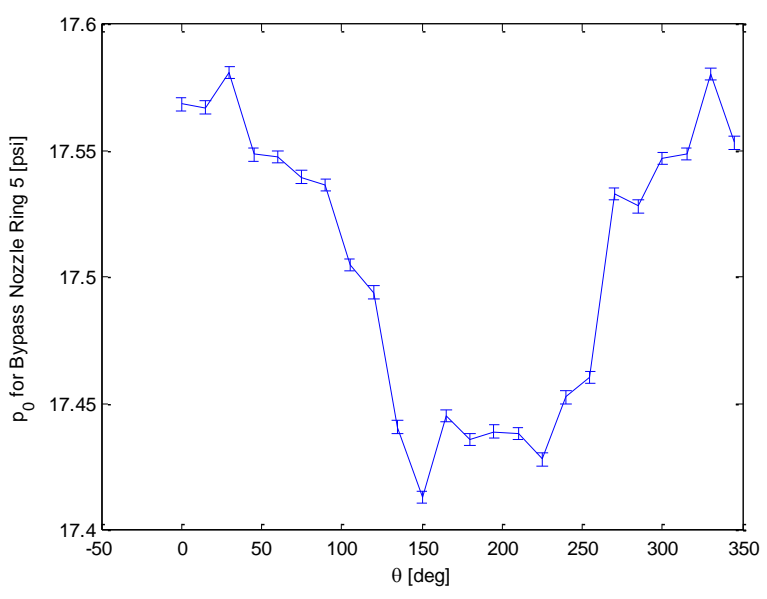

(b)

Figure B.1: Sample Error Bars for (a) AIP and (b) Bypass Nozzle Exit Plane Total Pressures

As shown in Figure B.1, the error bars on the total pressure measurements are small compared to the nominal total pressure measured. Alternatively, the uncertainty in a measurement can be expressed as a percentage of its value. A summary of the uncertainty and maximum uncertainty percentage for each of the measured values can be found in Table B.1. The maximum uncertainty percentage in the pressures measured at the AIP is $\pm 0.3 \%$, while the maximum uncertainty percentage in the pressures measured with the pressure scanner is $\pm 0.018 \%$. The maximum uncertainty percentage in the total temperatures recorded using the NI-9211 is \pm $0.64 \%$.

Table B.1: Measurement Uncertainty

\begin{tabular}{|c|c|c|}
\hline Parameter & Uncertainty & Percentage \\
\hline Pressure Scanner Pressures & $\pm 0.0025 \mathrm{psi}$ & $\pm 0.018 \%$ \\
\hline AIP Rake Pressures & $\pm 0.046 \mathrm{psi}$ & $\pm 0.3 \%$ \\
\hline Temperature & $\pm 2 \mathrm{~K}$ & $\pm 0.64 \%$ \\
\hline Fan Speed & $\pm 0.516 \mathrm{RPM}$ & $\pm 0.0041 \%$ \\
\hline Fuel Flow Rate & $\pm 1.13 \mathrm{GPH}$ & $\pm 1.03 \%$ \\
\hline
\end{tabular}

As shown in Table B.1, the uncertainty in each measurement is very small compared to its nominal value. Thus, it was concluded that these measurements can be used to adequately characterize the flow and engine operation.

To determine the effect of the uncertainties shown in Table B.1 on the performance calculations, each measurement was taken to its uncertainty extreme. The performance parameters were then recalculated using these extreme values. Every possible combination of uncertainty extremes was analyzed. The results were then analyzed to determine the maximum and minimum variation due to every possible combination of uncertainty extremes. These results are summarized in Table B.2. The values in the Clean and Distorted columns are the nominal values calculated assuming no uncertainty error. The values in the Uncertainty column are the maximum percentage variation from these nominal values. 
Table B.2: Calculated Values and Uncertainty

\begin{tabular}{|c|c|c|c|}
\hline Parameter & Clean & Distorted & Uncertainty \\
\hline Corrected Thrust [lbf] & 1058.44 & 894.26 & $\pm 0.92 \%$ \\
\hline Corrected TSFC [lbm/hr/lbf] & 0.7005 & 0.7975 & $\pm 1.94 \%$ \\
\hline Bypass Ratio & 3.0595 & 2.8503 & $\pm 2.03 \%$ \\
\hline Corrected Fan Speed [RPM] & 12,742 & 12,651 & $\pm 0.0032 \%$ \\
\hline Bellmouth Mass Flow [lbm/s] & 50.14 & 45.47 & $\pm 0.17 \%$ \\
\hline Fuel Flow [GPH] & 109.82 & 104.83 & $\pm 1.03 \%$ \\
\hline Core Nozzle Pressure Ratio & 0.9371 & 0.9444 & $\pm 0.3 \%$ \\
\hline Core Mach Number & 0.4013 & 0.389 & $\pm 1.75 \%$ \\
\hline Bypass Mach Number & 0.5847 & 0.5239 & $\pm 0.0074 \%$ \\
\hline Core Exit Velocity [m/s] & 220.19 & 214.68 & $\pm 1.82 \%$ \\
\hline Bypass Exit Velocity [m/s] & 202.6 & 182.63 & $\pm 0.28 \%$ \\
\hline
\end{tabular}

As shown in Table B.2, for every calculated performance parameter, the uncertainty is very small compared to its nominal value, giving confidence that these results are sufficiently accurate to support the conclusions of this thesis.

The uncertainty in the fan flow path efficiency and entropy production calculations was calculated using sensitivity coefficients and uncertainty in measured values. This method is summarized in Equations B.4 and B.5.

$$
\begin{gathered}
\theta_{i}=\frac{\partial R}{\partial x_{i}} \\
u_{R}= \pm\left(\sum_{i=1}^{n}\left(\theta_{i} u_{x i}\right)^{2}\right)^{\frac{1}{2}}
\end{gathered}
$$

In Equations B.4 and B.5, $\theta_{i}$ represents the sensitivity coefficient of variable $i$ for result $R$, and $u_{R}$ represents the uncertainty in result $R$. The results of this analysis for both entropy generation and fan flow path efficiency resulted in uncertainties at every measurement point for both clean and distorted tests. For the entire fan flow path entropy generation, the uncertainty varied from $\pm 6.92 \mathrm{~J} / \mathrm{kg} / \mathrm{K}$ to $\pm 7.24 \mathrm{~J} / \mathrm{kg} / \mathrm{K}$ with a mass-averaged uncertainty of $\pm 7.2 \mathrm{~J} / \mathrm{kg} / \mathrm{K}$ for the clean test, and from $\pm 6.84 \mathrm{~J} / \mathrm{kg} / \mathrm{K}$ to $\pm 7.34 \% \mathrm{~J} / \mathrm{kg} / \mathrm{K}$ with a mass-averaged uncertainty of $\pm 7.2 \mathrm{~J} / \mathrm{kg} / \mathrm{K}$ for the distorted test. For the fan flow path efficiency, the uncertainty varied from $\pm 1.4 \%$ to $\pm 5.2 \%$ with a mass-averaged uncertainty of $\pm 4.4 \%$ for the clean test, and from $\pm 0.7 \%$ to $\pm 6.6 \%$ with a mass-averaged uncertainty of $\pm 3.9 \%$ for the distorted test. These uncertainties were plotted as error bars for both the entire fan flow path entropy generation and fan flow path efficiency, as shown in 

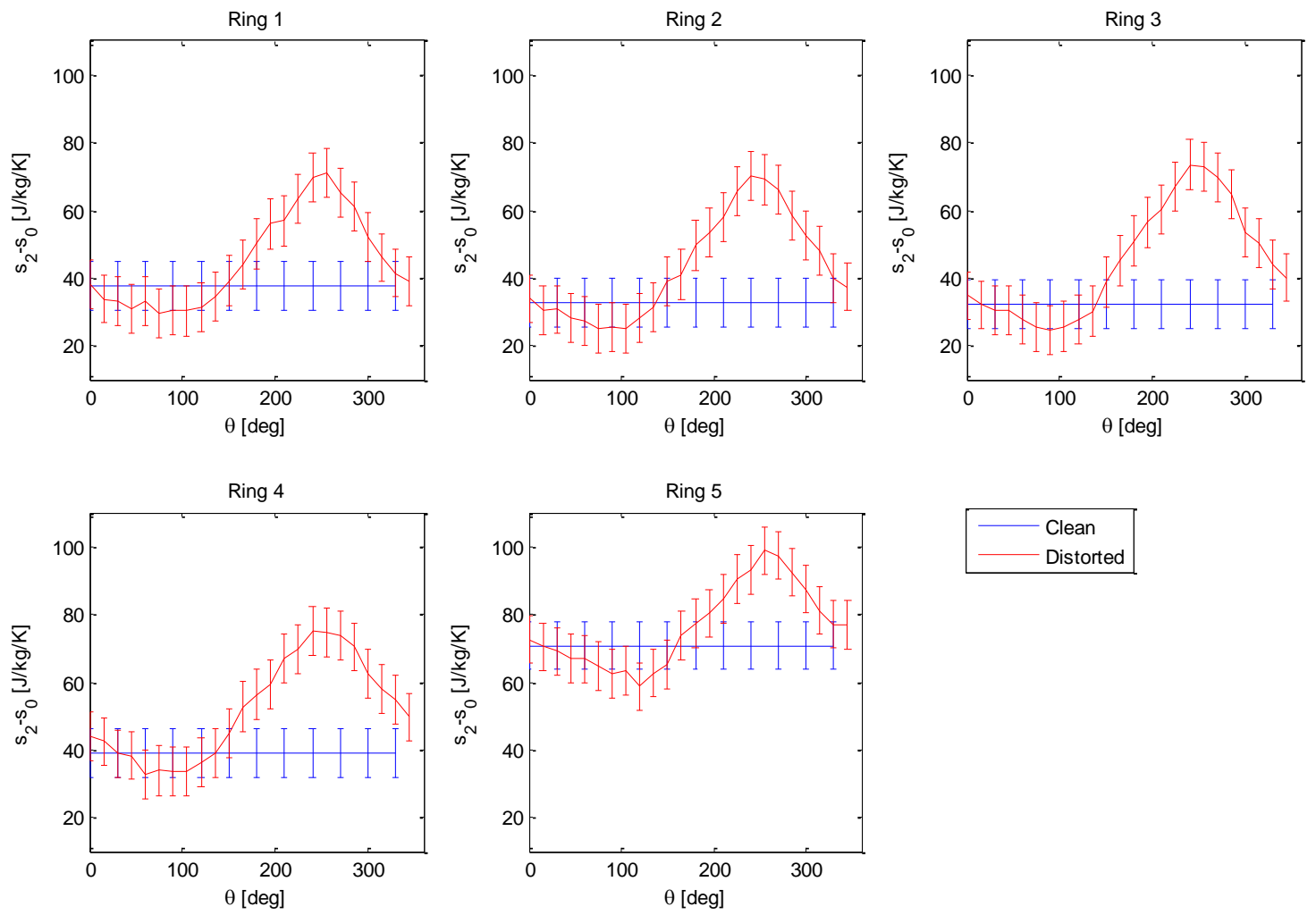

Figure B.2: Fan Flow Path Entropy Production Uncertainty for Each Ring
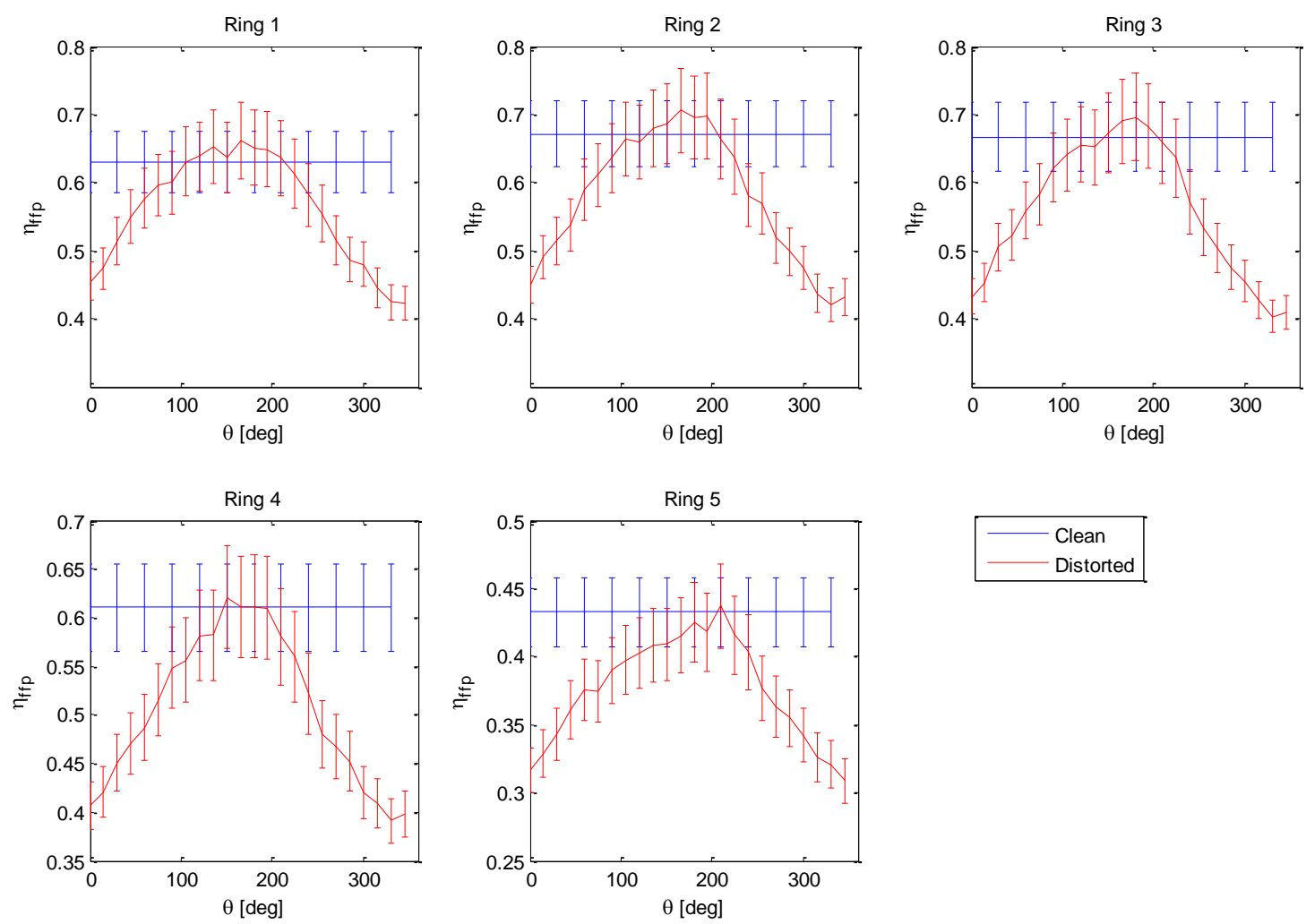

Figure B.3: Fan Flow Path Efficiency Uncertainty for Each Ring 
As shown in Figure B.2 and Figure B.3, the uncertainty in both fan flow path entropy generation and fan flow path efficiency is fairly substantial. The magnitude of the uncertainty is mainly due to the large uncertainty in the temperature measurements. An analysis was performed to determine how accurate the temperature measurements needed to be to create an uncertainty in fan flow path efficiency of $\pm 0.1 \%$. This analysis yielded that a temperature uncertainty of \pm 0.03 $\mathrm{K}$ would be required to reduce the fan flow path efficiency uncertainty to $\pm 0.1 \%$. This temperature measurement accuracy is unobtainable with standard thermocouples. Thus, if it is desired that the uncertainty in fan flow path entropy generation and fan flow path efficiency be reduced, a more accurate method of temperature measurement will be required.

An investigation into the effect of the assumption of constant specific heat capacity assumption used in the calculation of entropy generation was conducted to determine if this assumption was a significant contributor to the uncertainty in the entropy generation calculation. To analyze this assumption, the assumed constant specific heat capacity was interpolated at both the ambient temperature and the bypass nozzle exit temperature. Using these two specific heat capacity values, the magnitude of the mass-averaged entropy generation between ambient conditions and the bypass nozzle exit was compared for each value. The results of this investigation are shown in Table B.3.

Table B.3: Effect of Specific Heat Capacity Assumption

\begin{tabular}{|c|c|c|}
\hline \multirow{2}{*}{ Interpolation Temperature } & \multicolumn{2}{|c|}{ Mass-Averaged Entropy Generation $[\mathbf{J} / \mathbf{k g} / \mathbf{K}]$} \\
\cline { 2 - 3 } & Clean & Distorted \\
\hline Mean Total Temperature & 41.4486 & 51.2653 \\
\hline Bypass Exit Total Temperature & 41.5889 & 51.4116 \\
\hline Atmospheric Temperature & 41.4486 & 51.2652 \\
\hline
\end{tabular}

As shown in Table B.3, there is a minimal difference between the mass-averaged entropy generation between Stations 0 and 2 for the various temperatures used for interpolation of the specific heat capacity. The maximum percent difference from the nominal (mean total temperature interpolation) mass-averaged entropy generation was $0.34 \%$. Thus it was determined that the assumption of a constant specific heat capacity determined by the mean temperature between the two stations analyzed was a valid assumption. 


\section{Proof of Constant Total Temperature Across Distortion Screen}

$1^{\text {st }}$ Law of Thermodynamics

$$
\frac{\partial E}{\partial t}=\dot{Q}-\dot{W}+\dot{m} \Delta h_{0}
$$

Assume steady state, adiabatic screen, no work input

$$
0=\dot{m} \Delta h_{0}
$$

Definition of total enthalpy

$$
0=\dot{m} c_{\mathrm{p}} \Delta T_{0}
$$

Nonzero value of mass flow rate and specific heat yields

$$
0=\Delta T_{0}
$$

Therefore, there is no total temperature change across the distortion screen. 